\title{
Synthesis of isohexide diyne polymers and hydrogenation to their saturated polyethers
}

\author{
Ethan A. Gormong, Theresa M. Reineke,* \& Thomas R. Hoye* \\ Department of Chemistry, University of Minnesota, Minneapolis, Minnesota 55455, USA \\ * hoye@umn.edu , * treineke@umn.edu
}

\section{Contents of Supporting Information:}

I. General Experimental Protocols

II. Preparation and Characterization of Non-Polymeric compounds

a. $(3 R, 3 \mathrm{a} R, 6 S, 6 \mathrm{a} R)$-3,6-bis(Prop-2-yn-1-yloxy)hexahydrofuro[3,2-b]furan (2a)

b. $(3 R, 3 \mathrm{a} R, 6 R, 6 \mathrm{a} R)-3,6$-bis(Prop-2-yn-1-yloxy)hexahydrofuro[3,2-b]furan (2b)

c. $(3 S, 3 \mathrm{a} R, 6 S, 6 \mathrm{a} R)$-Hexahydrofuro[3,2-b]furan-3,6-diol (1c)

d. $(3 S, 3 \mathrm{a} R, 6 S, 6 \mathrm{a} R)-3,6$-bis(Prop-2-yn-1-yloxy)hexahydrofuro[3,2-b]furan (2c)

e. $(3 R, 3 \mathrm{a} R, 6 S, 6 \mathrm{a} R)-6$-(Prop-2-yn-1-yloxy)hexahydrofuro[3,2- $b]$ furan-3-ol (S1)

f. (3S,3aR,6R,6aR)-6-(prop-2-yn-1-yloxy)hexahydrofuro[3,2-b]furan-3-ol (S2)

g. $(3 R, 3 \mathrm{a} R, 6 S, 6 \mathrm{a} R)-6$-(Prop-2-yn-1-yloxy)hexahydrofuro[3,2-b]furan-3-yl acetate (3)

h. $\left(3 R, 3 \mathrm{a} R, 3^{\prime} R, 3 \mathrm{a}^{\prime} R, 6 S, 6 \mathrm{a} R, 6{ }^{\prime} S, 6 \mathrm{a}^{\prime} R\right)$-(Hexa-2,4-diyne-1,6-diylbis(oxy))bis(hexahydrofuro[3,2$b]$ furan-6,3-diyl) Diacetate (4)

i. $\quad(3 R, 3 \mathrm{a} R, 6 S, 6 \mathrm{a} R)-6-((3-I o d o p r o p-2-y n-1-y 1) o x y) h e x a h y d r o f u r o[3,2-b]$ furan-3-yl Acetate (5)

j. $\quad(3 R, 3 \mathrm{a} R, 6 S, 6 \mathrm{a} R)-3,6-b i s((3-I o d o p r o p-2-y n-1-y l) o x y)$ hexahydrofuro[3,2-b]furan (6)

k. $(3 R, 3 \mathrm{a} R, 6 S, 6 \mathrm{a} R)-3,6$-Dipropoxyhexahydrofuro[3,2-b]furan (7)

l. $\left(3 R, 3 \mathrm{a} R, 3^{\prime} R, 3 \mathrm{a}^{\prime} R, 6 S, 6 \mathrm{a} R, 66^{\prime} S, 6 \mathrm{a}^{\prime} R\right)$-6,6'-(Hexa-2,4-diyne-1,6-diylbis(oxy))bis(hexahydrofuro[3,2-b]furan-3-ol) (S3)

m. 1,6-bis (((3S,3a $R, 6 R, 6 \mathrm{a} R)$-6-(Prop-2-yn-1yloxy)hexahydrofuro[3,2-b]furan-3-yl)oxy)hexa2,4-diyne (2a-RR)

n. $(3 R, 3 \mathrm{a} R, 6 S, 6 \mathrm{a} R)-6$-(Formyloxy)hexahydrofuro[3,2- $b]$ furan-3-yl acetate (S5)

III. Preparation and Characterization of Polymers
a. $\operatorname{Poly}(2 a)$
b. $\operatorname{Poly}(2 b)$
c. $\operatorname{Poly}(2 \mathrm{c})$
d. $\operatorname{Poly}(2 \mathrm{a}-\mathrm{RR})$
e. Poly(2a)-[H]
f. $\operatorname{Poly}(2 \mathrm{~b})-[\mathrm{H}]$
g. $\operatorname{Poly}(2 \mathrm{c})-[\mathrm{H}]$

IV. DSC, TGA and SEC traces for Polymeric compounds

a. DSC thermograms for $\operatorname{poly}(2 \mathbf{a}-\mathbf{c}), \operatorname{poly}(2 \mathbf{a}-\mathbf{R R})$ and poly $(2 \mathbf{a}-\mathbf{c})-[\mathbf{H}]$

b. Brief discussion of the thermal properties (DSC) of Poly(2a-RR) 
c. TGA thermograms for $\operatorname{poly}(2 \mathbf{a}-\mathbf{c})$, poly $(2 \mathbf{a}-\mathbf{R R})$ and $\operatorname{poly}(2 \mathbf{a}-\mathbf{c})-[\mathbf{H}]$

d. SEC chromatograms for poly $(2 a-c)$, poly $(2 a-R R)$ and poly $(2 a-c)-[H]$

V. Model study for aerobic oxidation of 4

VI. References for Supporting Information

VII. Copies of ${ }^{1} \mathrm{H}$ and ${ }^{13} \mathrm{C}$ NMR Spectra

\begin{tabular}{|c|c|}
\hline 2a ${ }^{1} \mathrm{H} /{ }^{13} \mathrm{C}$ & S38 \\
\hline 2b ${ }^{1} \mathrm{H} /{ }^{13} \mathrm{C}$ & S41 \\
\hline $2 c^{1} \mathrm{H} /{ }^{13} \mathrm{C}$ & S43 \\
\hline $\mathbf{S 1}{ }^{1} \mathrm{H} /{ }^{13} \mathrm{C}$ & $\mathrm{S} 4$ \\
\hline S2 ${ }^{1} \mathrm{H} /{ }^{13} \mathrm{C}$ & $\mathrm{S} 4$ \\
\hline $3^{1} \mathrm{H} /{ }^{13} \mathrm{C}$ & $\mathrm{S} 4$ \\
\hline $4{ }^{1} \mathrm{H} /{ }^{13} \mathrm{C}$ & $\mathrm{S}$ \\
\hline $5{ }^{1} \mathrm{H} /{ }^{13} \mathrm{C}$ & S5 \\
\hline $6{ }^{1} \mathrm{H} /{ }^{13} \mathrm{C}$ & S5 \\
\hline $7{ }^{1} \mathrm{H} /{ }^{13} \mathrm{C}$ & $\mathrm{S}$ \\
\hline $\mathbf{S 3}{ }^{1} \mathrm{H} /{ }^{13} \mathrm{C}$ & $\mathrm{S}$ \\
\hline $\mathbf{2 a}-\mathbf{R} \mathbf{R}{ }^{1} \mathrm{H} /{ }^{13} \mathrm{C}$ & S61 \\
\hline S5 ${ }^{1} \mathrm{H} /{ }^{13} \mathrm{C}$ & S6 \\
\hline $\operatorname{Poly}(2 a){ }^{1} \mathrm{H} /{ }^{13} \mathrm{C}$ & S6 \\
\hline $\operatorname{Poly}(2 b){ }^{1} \mathrm{H} /{ }^{13} \mathrm{C}$ & S6 \\
\hline $\operatorname{Poly}(2 \mathrm{c}){ }^{1} \mathrm{H} /{ }^{13} \mathrm{C}$ & S69 \\
\hline $\operatorname{Poly}(2 \mathbf{a}-\mathbf{R R}){ }^{1} \mathrm{H} /{ }^{13} \mathrm{C}$ & $\mathrm{S}$ \\
\hline $\operatorname{Poly}(2 \mathbf{a})-[\mathbf{H}]{ }^{1} \mathrm{H} /{ }^{13} \mathrm{C}$ & S7 \\
\hline $\operatorname{Poly}(2 b)-[\mathbf{H}]{ }^{1} \mathrm{H} /{ }^{13} \mathrm{C}$ & S75 \\
\hline $\operatorname{Poly}(2 c)-[\mathbf{H}]{ }^{1} \mathrm{H} /{ }^{13} \mathrm{C}$ & $\mathrm{S}^{7}$ \\
\hline
\end{tabular}




\section{General Experimental Protocols}

Reagents: $N, N, N^{\prime}, N^{\prime}$-Tetramethylethylenediamine (Acros) and triethylamine (Fisher) were purified by vacuum distillation and stored over $\mathrm{KOH}$ pellets. Isosorbide was received as a gift chemical in excellent purity from Archer Daniels Midland Co. and was used without purification. Isomannide (98\%) was purchased from Acros and used as received. Propargyl bromide was purchased as a 9.0-9.2 M solution in toluene from TCI, stabilized by $0.3 \% \mathrm{MgO}$ and was filtered before use. Copper iodide was purchased from Sigma as a fine beige-colored powder and used without purification. Nickel (II) chloride hexahydrate (bright green) was purchased from Mallinckrodt and was ground into a fine powder with a mortar and pestle prior to use.

NMR: ${ }^{1} \mathrm{H}$ and ${ }^{13} \mathrm{C}$ NMR spectra were recorded on a Bruker Avance II or Avance III 500 spectrometer $\left({ }^{1} \mathrm{H}\right.$ recorded at $500 \mathrm{MHz},{ }^{13} \mathrm{C}$ at $\left.125 \mathrm{MHz}\right) .{ }^{1} \mathrm{H}$ NMR chemicals shift using, $\mathrm{CDCl}_{3}$ and DMSO- $d_{6}$ are referenced to TMS at $0.00 \mathrm{ppm}$ and $\mathrm{CHD}_{2} \mathrm{SOCD}_{3}$ at $\delta 2.50 \mathrm{ppm}$, respectively. Multiplets are reported as s (singlet), d (doublet), t (triplet), m (multiplet), and nfom (non-firstorder multiplet).

ATR-FTIR: An ATR-FTIR instrument (Bruker Alpha Platinum), fitted with a diamond singlebounce crystal, was used to record FT infrared spectra. Measurements were typically recorded with sixteen scans and a four second acquisition time. Samples were deposited as thin films directly onto the diamond window; solids were then also compressed with a pressure anvil.

MPLC (medium pressure liquid chromatography) was performed at ca. 25-200 psi on handpacked silica gel columns $\left(25-35 \mu \mathrm{m}, 40 \AA\right.$ pore size) at a flow rate of ca. $9 \mathrm{~mL} \mathrm{~min}^{-1}$. The instrument comprised a Waters HPLC pump and a Waters R401 differential refractive index (RI) detector. The samples were introduced onto the column after as a solution in DCM, and various ratios of hexanes and ethyl acetate were used as the mobile phase. Flash chromatography was carried out on columns packed with a larger size of silica gel particles $(40-63 \mu \mathrm{m})$.

GC-MS: Low-resolution mass spectra were obtained in electron ionization mode using an Agilent $5975 \mathrm{MSD}$ at $70 \mathrm{eV}$. This was connected to an Agilent 6953 gas chromatograph outfitted with an Agilent HP-5 column $(0.25 \mu \mathrm{m}$ film thickness, $15 \mathrm{~m}$ length $\times 0.32 \mathrm{~mm}$ ID) .

HRMS: High-resolution mass spectra (HRMS) were recorded using ESI ionization on a Thermo Orbitrap Velos instrument. An external calibrant (PierceTM LTQ) was used. Samples were directly injected into the ion source.

SEC-MALS: Polymer molecular weight $\left(M_{\mathrm{w}}, M_{\mathrm{n}}\right)$ and dispersity $(\nexists)$ were determined by size exclusion chromatography (SEC) using an Agilent 1260 Infinity liquid chromatograph. Dimethylformamide (DMF) containing $0.05 \mathrm{M} \mathrm{LiBr}$ was used as the mobile phase. The flow rate was $1.0 \mathrm{~mL} \mathrm{~min}{ }^{-1}$ at $40{ }^{\circ} \mathrm{C}$. Wyatt Optilab T-rEX refractive index and Wyatt Dawn Heleos II 
multiangle laser light scattering (MALS) detectors were used. The latter collected scattering from 18 detector angles ranging from $10^{\circ}$ to $160^{\circ}$ and used a $663.6 \mathrm{~nm}$ laser.

TGA: TGA (TA Instruments Q500) sample sizes ranged from 4-10 $\mathrm{mg}$. The samples were heated under a mixture of $\mathrm{N} 2$ and air at a heating rate of $10^{\circ} \mathrm{C} \mathrm{min}^{-1}$ from r.t. to $550{ }^{\circ} \mathrm{C}$.

DSC: was performed on a TA Instruments Q-1000. Samples were measured in hermetically sealed aluminum pans. Sample sizes ranged from 2-8 $\mathrm{mg}$. Each diyne polymer sample, poly(2a)poly(2c) and poly(2a-RR), was equilibrated to $-50{ }^{\circ} \mathrm{C}$ and ramped at $20{ }^{\circ} \mathrm{C} \mathrm{min}^{-1}$ to the upper temperature limit $\left(150\right.$ or $\left.200{ }^{\circ} \mathrm{C}\right)$. Glass transition temperatures were measured during the first and only heating ramp, due to aerobic oxidation at elevated temperatures. The hydrogenated diyne polymers poly $(\mathbf{2 a}-\mathbf{c})-[\mathbf{H}]$ were equilibrated to $-65{ }^{\circ} \mathrm{C}$ and ramped at $10{ }^{\circ} \mathrm{C} \mathrm{min}^{-1}$ to the upper temperature limit $\left(100^{\circ} \mathrm{C}\right)$. Measurements were recorded on the second heating ramp. 


\section{Preparation and Characterization of non-Polymeric compounds}

\section{(3R,3aR,6S,6aR)-3,6-bis(Prop-2-yn-1-yloxy)hexahydrofuro[3,2-b]furan (2a).}

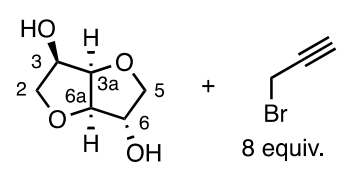

1 a

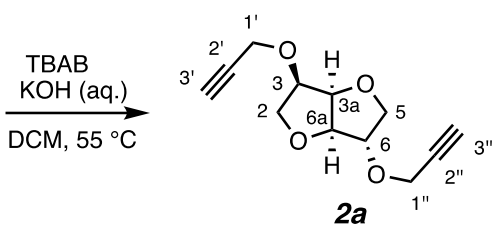

The following is a representative procedure for the production of the isohexide bis-propargyl ethers $\mathbf{2 a - 2 c}$. Isosorbide (3.00 g, $20.5 \mathrm{mmol})$ was dissolved in a potassium hydroxide solution (13.8 g, $247 \mathrm{mmol}$ in 50 $\mathrm{mL}$ DI water) in a round-bottom flask equipped with a stir bar. A $9.0 \mathrm{M}$ solution of propargyl bromide in toluene $(18.3 \mathrm{~mL}, 164 \mathrm{mmol})$ was dissolved in $50 \mathrm{~mL}$ of dichloromethane, which was added to the flask, followed by addition of tetrabutylammonium bromide $(660 \mathrm{mg}, 2.1 \mathrm{mmol})$. This biphasic mixture was stirred under a reflux condenser at $55{ }^{\circ} \mathrm{C}$ for $24 \mathrm{~h}$. The aqueous phase was extracted with $2 \times 50 \mathrm{~mL}$ of dichloromethane, and the combined organic layers were washed with satd $\mathrm{NH}_{4} \mathrm{Cl}(2 \mathrm{x})$ and brine (2x), dried over anhyd $\mathrm{MgSO}_{4}$, filtered, and concentrated in vacuo. The residue was purified by flash chromatography on silica gel (3:1 hexanes/EtOAc). The resulting product 2a was obtained in $66 \%$ yield (3.01 g) as a yellow oil. The ${ }^{1} \mathrm{H}$ NMR data corresponded closely to those reported. ${ }^{1}$

${ }^{1}$ H NMR (500 MHz, DMSO- $d_{6}$ ): $\delta 4.58$ (dd, $\left.J=4.6,4.6 \mathrm{~Hz}, 1 \mathrm{H}, H 3 \mathrm{a}\right), 4.48$ (dd, $\left.J=4.6,0.9 \mathrm{~Hz}, 1 \mathrm{H}, H 6 \mathrm{a}\right)$, $4.27\left(\mathrm{dd}, J=15.8,2.4 \mathrm{~Hz}, 1 \mathrm{H}, \mathrm{C} 1{ }^{\prime} H_{\mathrm{a}} \mathrm{H}_{\mathrm{b}}\right), 4.22\left(\mathrm{~d}, J=2.5 \mathrm{~Hz}, 2 \mathrm{H}, \mathrm{C} 1^{\prime}{ }^{\prime} H_{2}\right), 4.18(\mathrm{dd}, J=15.8,2.4 \mathrm{~Hz}, 1 \mathrm{H}$, $\mathrm{Cl}^{\prime} \mathrm{H}_{\mathrm{a}} H_{\mathrm{b}}$ ), 4.14 (ddd, $J=7.2,6.5,4.7 \mathrm{~Hz}, 1 \mathrm{H}, H 3$ ), 4.07 (brd, $\left.J=4.1 \mathrm{~Hz}, 1 \mathrm{H}, H 6\right), 3.88$ (ddd, $J=10.3,1.2$, $\left.1.2 \mathrm{~Hz}, 1 \mathrm{H}, \mathrm{C} 5 \mathrm{H}_{\mathrm{a}} \mathrm{H}_{\mathrm{b}}\right), 3.82\left(\mathrm{dd}, J=8.8,6.5 \mathrm{~Hz}, 1 \mathrm{H}, \mathrm{C} 2 \mathrm{H}_{\mathrm{a}} \mathrm{H}_{\mathrm{b}}\right), 3.75\left(\mathrm{dd}, J=10.3,3.8 \mathrm{~Hz}, 1 \mathrm{H}, \mathrm{C} 5 \mathrm{H}_{\mathrm{a}} H_{\mathrm{b}}\right), 3.48$ (t, $J=2.4 \mathrm{~Hz}, 1 \mathrm{H}, H 3$ ' or $H 3$ ''), 3.47 (t, $J=2.4 \mathrm{~Hz}, 1 \mathrm{H}, H 3$ ' or $H 3$ ''), and 3.45 (dd, $J=8.8,7.2 \mathrm{~Hz}, 1 \mathrm{H}$, $\left.\mathrm{C} 2 \mathrm{H}_{\mathrm{a}} H_{\mathrm{b}}\right)$.

${ }^{1}$ H NMR (500 MHz, $\mathrm{CDCl}_{3}$ ): $\delta 4.71(\mathrm{dd}, J=4.5,4.5 \mathrm{~Hz}, 1 \mathrm{H}, H 3 \mathrm{a}), 4.60$ (dd, $\left.J=4.3,0.9 \mathrm{~Hz}, 1 \mathrm{H}, H 6 \mathrm{a}\right)$, $4.37\left(\mathrm{dd}, J=16.0,2.4 \mathrm{~Hz}, 1 \mathrm{H}, \mathrm{C} 1^{\prime} H_{\mathrm{a}} \mathrm{H}_{\mathrm{b}}\right), 4.31\left(\mathrm{dd}, J=15.9,2.4 \mathrm{~Hz}, 1 \mathrm{H}, \mathrm{C} 1{ }^{\prime} \mathrm{H}_{\mathrm{a}} H_{\mathrm{b}}\right), 4.30$ (ddd, $J=7.2,6.5$, $4.7 \mathrm{~Hz}, 1 \mathrm{H}, H 3),\left(\mathrm{dd}, J=16.0,2.4 \mathrm{~Hz}, 1 \mathrm{H}, \mathrm{C}^{\prime} H_{\mathrm{a}} \mathrm{H}_{\mathrm{b}}\right), 4.07$ (brd, $\left.J=4.1 \mathrm{~Hz}, 1 \mathrm{H}, H 6\right), 3.88$ (ddd, $J=10.3$, $\left.1.2,1.2 \mathrm{~Hz}, 1 \mathrm{H}, \mathrm{C} 5 H_{\mathrm{a}} \mathrm{H}_{\mathrm{b}}\right), 3.82\left(\mathrm{dd}, J=8.8,6.5 \mathrm{~Hz}, 1 \mathrm{H}, \mathrm{C} 2 \mathrm{H}_{\mathrm{a}} \mathrm{H}_{\mathrm{b}}\right), 3.75\left(\mathrm{dd}, J=10.3,3.8 \mathrm{~Hz}, 1 \mathrm{H}, \mathrm{C} \mathrm{H}_{\mathrm{a}} H_{\mathrm{b}}\right.$ ), 3.67 (dd, $\left.J=8.9,7.7 \mathrm{~Hz}, 1 \mathrm{H}, \mathrm{C}_{2} \mathrm{H}_{\mathrm{a}} H_{\mathrm{b}}\right), 2.49$ (t, $J=2.4 \mathrm{~Hz}, 1 \mathrm{H}, H 3^{\prime}$ or $\left.H 3^{\prime \prime}\right)$, and 2.48 (t, $J=2.4 \mathrm{~Hz}, 1 \mathrm{H}$, $H 3$ ' or $H 3$ '').

${ }^{13}$ C NMR (125 MHz, DMSO-d $d_{6}$ ): $\delta 85.6$ (C6a), 83.0 (C6), 80.6 (C2' or C2'”), 80.5 (C2' or C2'’), 80.2 (C3a), 78.9 (C3), 77.83 (C3' or C3'’), 77.80 (C3' or C3'), 72.8 (C5), 69.9 (C2), 57.3 (C1'), and 56.5 (C1',).

FT-IR (thin film): 3281, 3274, 2941, 2875, 2116, 1732, 1243, 1062, and $634 \mathrm{~cm}^{-1}$.

GC-MS $\left.50{ }^{\circ} \mathrm{C} / 2.0 \mathrm{~min} / 20{ }^{\circ} \mathrm{C} \mathrm{min}-1 / 250{ }^{\circ} \mathrm{C}\right) t R=7.93 \mathrm{~min}$; MS $[70 \mathrm{eV}, \mathrm{m} / z$ (rel int)]: $222(1, \mathrm{M}+), 183(2$, $\left.\mathrm{M}+-\mathrm{CH}_{2} \mathrm{C} \equiv \mathrm{CH}\right), 82\left(33, \mathrm{C}_{5} \mathrm{H}_{6} \mathrm{O}_{2}^{+}\right)$, and $69\left(100, \mathrm{C}_{4} \mathrm{H}_{5} \mathrm{O}^{+}\right.$, protonated furan $)$. 
$(3 R, 3 a R, 6 R, 6 a R)-3,6-b i s(P r o p-2-y n-1-y l o x y) h e x a h y d r o f u r o[3,2-b]$ furan (2b)

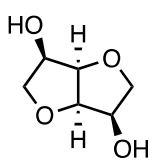

$1 b$

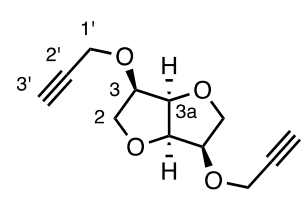

$2 b$

Diyne 2b was prepared by the same method used to make 2a, starting with $3.0 \mathrm{~g}$ of isomannide (1) $\mathbf{b}$ ). The solid crude product was purified by recrystallization in 3:1 hexanes/EtOAc, filtered, washed with cold hexanes, and dried in vacuo, yielding $\mathbf{2 b}$ as light-yellow needle-like crystals ( $2.55 \mathrm{~g}, 56 \%)$. Characterization data closely matched those previously reported. ${ }^{1}$

${ }^{1}$ H NMR (500 MHz, DMSO- $d_{6}$ ): $\delta 4.53$ (nfom, including $J_{3 a / 6 a}=4.6 \mathrm{~Hz}, H 3 \mathrm{a}$ ), 4.26 (dd, $J=15.8,2.4 \mathrm{~Hz}$, $\left.2 \mathrm{H}, \mathrm{C} 1{ }^{\prime} H_{\mathrm{a}} \mathrm{H}_{\mathrm{b}}\right), 4.16\left(\mathrm{dd}, J=15.8,2.4 \mathrm{~Hz}, 2 \mathrm{H}, \mathrm{C} 1{ }^{\prime} \mathrm{H}_{\mathrm{a}} H_{\mathrm{b}}\right), 4.13$ (dddd, $J=8.4,6.8,3.6,1.6 \mathrm{~Hz}, 2 \mathrm{H}, H 3$ ), $3.91\left(\mathrm{dd}, J=8.4,6.8, \mathrm{~Hz}, 2 \mathrm{H}, \mathrm{C} 2 H_{\mathrm{a}} \mathrm{H}_{\mathrm{b}}\right), 3.47$ (t, $J=2.4 \mathrm{~Hz}, 2 \mathrm{H}, H 3$ '), and 3.46 (dd, $J=8.4,8.4 \mathrm{~Hz}, 2 \mathrm{H}$, $\left.\mathrm{C} 2 \mathrm{H}_{\mathrm{a}} H_{\mathrm{b}}\right)$.

${ }^{13}$ C NMR $\left(125 \mathrm{MHz}, \mathrm{DMSO}-d_{6}\right): \delta 80.6,80.2,78.9,77.9,70.7$, and 57.3.

FT-IR (thin film): 3232, 2114, 1441, 1327, 1095, 1022, 914, 844, $672 \mathrm{~cm}^{-1}$.

GC-MS $\left.50{ }^{\circ} \mathrm{C} / 2.0 \mathrm{~min} / 20{ }^{\circ} \mathrm{C} \mathrm{min}-1 / 250{ }^{\circ} \mathrm{C}\right) t R=7.93 \mathrm{~min} ; \mathrm{MS}[70 \mathrm{eV}, \mathrm{m} / \mathrm{z}$ (rel int) $]: 183\left(2,-\mathrm{CH}_{2} \mathrm{C} \equiv \mathrm{CH}\right)$, $82\left(39, \mathrm{C}_{5} \mathrm{H}_{6} \mathrm{O}_{2}^{+}\right)$, and $69\left(100, \mathrm{C}_{4} \mathrm{H}_{5} \mathrm{O}^{+}\right.$, protonated furan).

mp: $90-93{ }^{\circ} \mathrm{C}$. 


\section{$(3 S, 3 a R, 6 S, 6 a R)$-Hexahydrofuro[3,2-b]furan-3,6-diol (1c)}

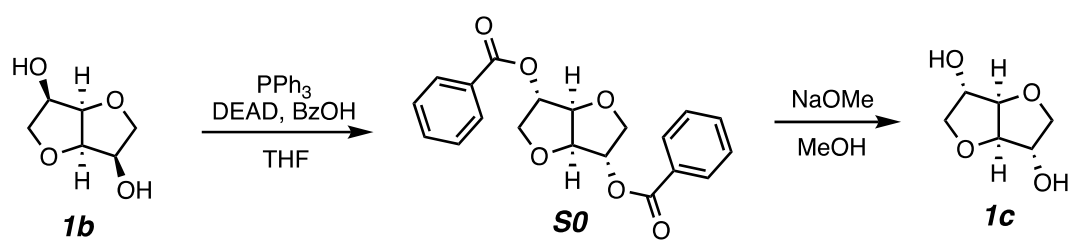

Isoidide (1c) was prepared according to a procedure adapted from Rajput and Chikkali. ${ }^{2}$ Triphenylphosphine $(3.59 \mathrm{~g}, 13.7 \mathrm{mmol})$, benzoic acid $(1.67 \mathrm{~g}, 13.7 \mathrm{mmol})$, and isomannide $(\mathbf{1 b}, 1.00 \mathrm{~g}$, $6.9 \mathrm{mmol}$ ) were dissolved in $20 \mathrm{~mL}$ of tetrahydrofuran in an oven-dried round-bottom flask equipped with a stir bar and sealed with a septum. A solution of diethyl azodicarboxylate $(2.15 \mathrm{~mL}, 13.9 \mathrm{mmol})$ in $20 \mathrm{~mL}$ of tetrahydrofuran was added to the round-bottom flask dropwise over a period of 4 hours via syringe pump. The mixture was allowed to stir for 16 hours, at which point additional portions of triphenylphosphine $(0.36$ $\mathrm{g}, 1.4 \mathrm{mmol})$, diethyl azodicarboxylate $(0.22 \mathrm{~mL}, 1.4 \mathrm{mmol})$, and benzoic acid $(0.17 \mathrm{~g}, 1.4 \mathrm{mmol})$ were added simultaneously to the flask, and the mixture was allowed to stir for an additional 3 hours.

The solution was concentrated in vacuo, and the resulting crude white solid was triturated in 3:1 hexanes/EtOAc, and the byproduct diethyl hydrazine-1,2-dicarboxylate (confirmed by GC-MS) was removed by filtration. The filtrate was concentrated and passed through plugs of silica and basic alumina, removing any excess benzoic acid, benzoic anhydride, and triphenylphosphine oxide. The crude filtrate contained the exo-dibenzoate $\mathbf{S 0}$ and some excess triphenyl phosphine. This solution was concentrated in vacuo, yielding an off-white solid, which was used in the next sequence without further purification. A portion was subjected to MPLC (3:1 hexanes/EtOAc) to yield a more pure sample of S0 whose ${ }^{1} \mathrm{H}$ NMR data were fully consistent with those reported. ${ }^{2}$

The crude exo-dibenzoate S0, ca. $1.6 \mathrm{~g}$, was suspended in $30 \mathrm{~mL}$ of methanol in a round bottom flask and stirred vigorously at $60{ }^{\circ} \mathrm{C}$ for $2 \mathrm{~h}$ to fully dissolve the diester. This solution was cooled slowly under vigorous stirring to $\sim 30-40{ }^{\circ} \mathrm{C}$. Sodium methoxide ( $710 \mathrm{mg}, 3.0$ equiv., $13 \mathrm{mmol}$ ) was added to the tube, which was sealed with a septum and allowed to stir for 3 hours at ambient temperature. The reaction was quenched with Dowex ${ }^{\circledR} 50 \mathrm{~W}-\mathrm{X} 1 \mathrm{resin}$ beads, which was filtered through Celite after stirring for $30 \mathrm{~min}$. The methanolic solution was concentrated in vacuo and poured into $40 \mathrm{~mL}$ of DI water and extracted $3 \mathrm{x}$ with XX mL of dichloromethane (GC-MS analysis of the dichloromethane extract revealed that the residual triphenyl phosphine and methyl benzoate were no longer present). The water-methanol layer was concentrated in vacuo and then lyophilized to afford $612 \mathrm{mg}$ isoidide $1 \mathrm{c}$ in (61\% yield over two steps). 
(3S,3aR,6S,6aR)-3,6-bis(Prop-2-yn-1-yloxy)hexahydrofuro[3,2-b]furan (2c)

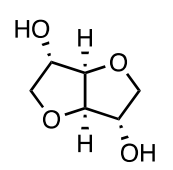

$1 c$

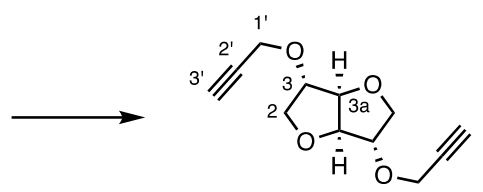

$2 c$

The residue was purified by MPLC (3:1 hexanes/EtOAc) and concentrated in vacuo, yielding $\mathbf{2 c}$ as a lightyellow solid in $55 \%$ yield $(450 \mathrm{mg})$. Characterization data closely matched those previously reported. ${ }^{1}$

${ }^{1}$ H NMR (500 MHz, DMSO- $\left.d_{6}\right): \delta 4.67$ (br s, 2H, H3a), 4.24 (dd, $J=16.0,2.4 \mathrm{~Hz}, 2 \mathrm{H}, \mathrm{Cl}^{\prime} H_{\mathrm{a}} \mathrm{H}_{\mathrm{b}}$ ), 4.23 (dd, $J=16.1,2.4 \mathrm{~Hz}, 2 \mathrm{H}, \mathrm{Cl}{ }^{\prime} \mathrm{H}_{\mathrm{a}} H_{b}$ ), 4.07 (dd, $J=3.8,1.8 \mathrm{~Hz}, 2 \mathrm{H}, H 3$ ), 3.80 (dd, $J=10.2,1.6 \mathrm{~Hz}, 2 \mathrm{H}$, $\left.\mathrm{C} 2 H_{\mathrm{a}} \mathrm{H}_{\mathrm{b}}\right), 3.71\left(\mathrm{dd}, J=10.2,3.9 \mathrm{~Hz}, 2 \mathrm{H}, \mathrm{C}_{2} \mathrm{H}_{\mathrm{a}} H_{\mathrm{b}}\right)$, and 3.48 (t, $J=2.4 \mathrm{~Hz}, 2 \mathrm{H}, H 3$ ').

${ }^{13}$ C NMR $\left(125 \mathrm{MHz}, \mathrm{DMSO}-d_{6}\right): \delta 84.9,82.5,80.6,77.9,71.7$, and 56.6.

FT-IR (thin film): 3251, 2942, 2116, 1731 (w), 1453, 1081, 1022, 839, 780, 672, and $477 \mathrm{~cm}^{-1}$.

GC-MS $\left.50{ }^{\circ} \mathrm{C} / 2.0 \mathrm{~min} / 20{ }^{\circ} \mathrm{C} \min -1 / 250{ }^{\circ} \mathrm{C}\right) t R=7.82 \mathrm{~min}$; MS $[70 \mathrm{eV}, \mathrm{m} / z$ (rel int)]: $222(3, \mathrm{M}+), 183$ $\left(1,-\mathrm{CH}_{2} \mathrm{C} \equiv \mathrm{CH}\right), 124\left(14, \mathrm{C}_{7} \mathrm{H}_{8} \mathrm{O}_{2}^{+}\right), 82\left(33, \mathrm{C}_{5} \mathrm{H}_{6} \mathrm{O}_{2}{ }^{+}\right)$, and $69\left(100, \mathrm{C}_{4} \mathrm{H}_{5} \mathrm{O}^{+}\right.$, protonated furan).

mp: $51-53{ }^{\circ} \mathrm{C}$. 
(3R,3aR,6aR,6S)-6-(Prop-2-yn-1-yloxy)hexahydrofuro[3,2-b]furan-3-ol (S1) and (3S,3aR,6aR,6R)-6-(Prop-2-yn-1-yloxy)hexahydrofuro[3,2-b]furan-3-ol (S2)

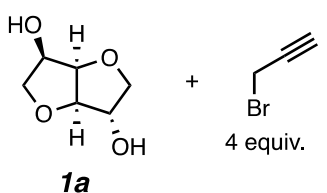

$1 a$
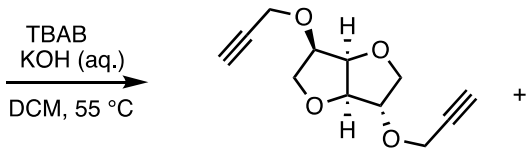

$2 a$

$29 \%$
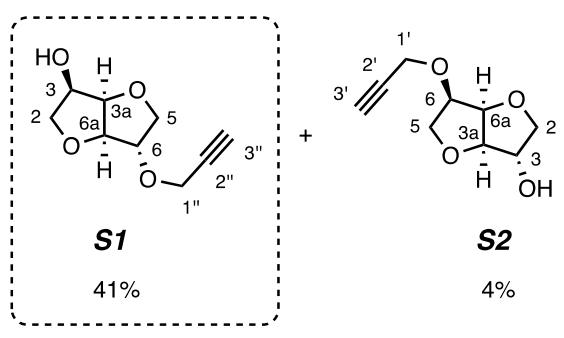

Isosorbide (1a, $3.00 \mathrm{~g}, 20.5 \mathrm{mmol})$ was dissolved in a potassium hydroxide solution (13.8 $\mathrm{g}, 30.8 \mathrm{mmol}$ in $30 \mathrm{~mL}$ DI water) in a round-bottom flask equipped with a stir bar. Tetrabutylammonium bromide (660 mg, $2.1 \mathrm{mmol}$ ) was added to the flask. This biphasic mixture was stirred under a reflux condenser at $55{ }^{\circ} \mathrm{C}$. A $9.0 \mathrm{M}$ solution of propargyl bromide in toluene $(9.1 \mathrm{~mL}, 82 \mathrm{mmol})$ was added to the flask via syringe pump over $4 \mathrm{~h}$, and the mixture was stirred for an additional $20 \mathrm{~h}$ under reflux. The aqueous phase was extracted with $2 \times 50 \mathrm{~mL}$ of dichloromethane, and the combined organic layers were washed with satd $\mathrm{NH}_{4} \mathrm{Cl}(2 \mathrm{x})$ and brine (2x), dried over anhyd $\mathrm{MgSO}_{4}$, filtered, and concentrated in vacuo. The oily residue was purified by MPLC (3:1 hexanes/EtOAc) to give, in order of elution, 2a (1.32 g, 29\%), S1 (1.55 g, 41\%), and S2 (150 $\mathrm{mg}, 4 \%$ ), whose ${ }^{1} \mathrm{H}$ NMR data was fully consistent with those reported. ${ }^{3}$

\section{Characterization Data for S1}

${ }^{1}$ H NMR (500 MHz, DMSO- $d_{6}$ ): $\delta 4.83$ (br d, $\left.J=6 \mathrm{~Hz}, 1 \mathrm{H}, \mathrm{C} 3 \mathrm{OH}\right), 4.44$ (ddd, $J=4.6,0.9,0.9 \mathrm{~Hz}, 1 \mathrm{H}$, $H 6 \mathrm{a}), 4.34$ (dd, $J=4.7,4.7 \mathrm{~Hz}, 1 \mathrm{H}, H 3 \mathrm{a}), 4.22$ (dd, $\left.J=16.2,2.4 \mathrm{~Hz}, 1 \mathrm{H}, \mathrm{C} 1{ }^{\prime}{ }^{\prime} H_{\mathrm{a}} \mathrm{H}_{\mathrm{b}}\right), 4.21$ (dd, $J=16.2,2.4$ $\mathrm{Hz}, 1 \mathrm{H}, \mathrm{C1}$ ' ' $\mathrm{H}_{\mathrm{a}} H_{\mathrm{b}}$ ), 4.10 (dddd, $J=7.7,6.4,6.4,4.8 \mathrm{~Hz}, 1 \mathrm{H}, H 3$ ), 4.07 (dddd, $J=3.8,1.5,1.2,0.6 \mathrm{~Hz}, 1 \mathrm{H}$, $H 6$ ), 3.87 (ddddd, $J=10.2,1.4,0.9,0.5,0.5 \mathrm{~Hz}, 1 \mathrm{H}, \mathrm{C} 5 H_{\mathrm{a}} \mathrm{H}_{\mathrm{b}}$ ), 3.77 (dd, $J=10.2,3.9 \mathrm{~Hz}, 1 \mathrm{H}, \mathrm{C} \mathrm{H}_{\mathrm{a}} H_{b}$ ), $3.72\left(\mathrm{dd}, J=8.3,6.5,0.3 \mathrm{~Hz}, 1 \mathrm{H}, \mathrm{C} 2 H_{a} \mathrm{H}_{\mathrm{b}}\right), 3.45(\mathrm{t}, J=2.4 \mathrm{~Hz}, 1 \mathrm{H}, H 3$ ' '), and 3.30 (ddd, $J=8.3,7.7,0.6$ $\left.\mathrm{Hz}, 1 \mathrm{H}, \mathrm{C} 2 \mathrm{H}_{\mathrm{a}} H_{\mathrm{b}}\right)$.

${ }^{13}$ C NMR (125 MHz, DMSO- $\left.d_{6}\right): \delta 85.3,83.4,81.9,80.6,77.9,72.7,72.5,71.5$, and 56.5.

FT-IR (thin film): 3432 (v br), 3275, 2940, 2875, 2116, 1047, 829, and $648 \mathrm{~cm}^{-1}$.

GC-MS $\left.50{ }^{\circ} \mathrm{C} / 2.0 \mathrm{~min} / 20^{\circ} \mathrm{C} \mathrm{min}^{-1} / 250{ }^{\circ} \mathrm{C}\right) t_{R}=6.59 \mathrm{~min}$; MS $\left[70 \mathrm{eV}, \mathrm{m} / z\right.$ (rel int)]: $184\left(10, \mathrm{M}^{+}\right), 124$ $\left(20, \mathrm{C}_{7} \mathrm{H}_{8} \mathrm{O}_{2}^{+}\right), 82\left(42, \mathrm{C}_{5} \mathrm{H}_{6} \mathrm{O}_{2}^{+}\right), 69\left(100, \mathrm{C}_{4} \mathrm{H}_{5} \mathrm{O}^{+}\right.$, protonated furan).

mp: $32-34{ }^{\circ} \mathrm{C}$.

\section{Characterization Data for S2}

${ }^{1}$ H NMR (500 MHz, DMSO- $\left.d_{6}\right): \delta 5.15(\mathrm{~d}, J=3.7 \mathrm{~Hz}, 1 \mathrm{H}, \mathrm{C} 3 \mathrm{OH}), 4.58(\mathrm{dd}, J=4.3,4.4 \mathrm{~Hz}, 1 \mathrm{H}, H 6 \mathrm{a})$, 4.28 (brd, $J=4.8 \mathrm{~Hz}, 1 \mathrm{H}, H 3 \mathrm{a}), 4.26\left(\mathrm{dd}, J=15.8,2.4 \mathrm{~Hz}, 1 \mathrm{H}, \mathrm{Cl}^{\prime} H_{\mathrm{a}} \mathrm{H}_{\mathrm{b}}\right), 4.18(\mathrm{dd}, J=15.8,2.4 \mathrm{~Hz}, 1 \mathrm{H}$, $\mathrm{Cl}^{\prime} \mathrm{H}_{\mathrm{a}} H_{\mathrm{b}}$ ), 4.14 (ddd, $\left.J=7.6,6.7,4.6 \mathrm{~Hz}, 1 \mathrm{H}, H 6\right), 4.04$ (nfom, $\left.1 \mathrm{H}, H 3\right), 3.81$ (dd, $J=8.6,6.7 \mathrm{~Hz}, 1 \mathrm{H}$, $\left.\mathrm{C} 5 H_{\mathrm{a}} \mathrm{H}_{\mathrm{b}}\right), 3.73\left(\mathrm{dd}, J=9.5,3.4 \mathrm{~Hz}, 1 \mathrm{H}, \mathrm{C} 2 \mathrm{H}_{\mathrm{a}} \mathrm{H}_{\mathrm{b}}\right), 3.70$ (brd, $\left.J=9.3 \mathrm{~Hz}, 1 \mathrm{H}, \mathrm{C}_{2} \mathrm{H}_{\mathrm{a}} H_{\mathrm{b}}\right), 3.45(\mathrm{t}, J=2.4 \mathrm{~Hz}$, $\left.1 \mathrm{H}, H 3^{\prime}\right)$, and $3.39\left(\mathrm{dd}, J=8.7,7.6 \mathrm{~Hz}, 1 \mathrm{H}, \mathrm{C} 5 \mathrm{H}_{\mathrm{a}} H_{\mathrm{b}}\right)$.

${ }^{13}$ C NMR (125 MHz, DMSO- $\left.d_{6}\right): \delta 88.6,80.7,79.9,79.2,77.8,75.9,75.8,69.6$, and 57.2.

FT-IR (thin film): 3409 (v br), 3268, 2942, 2875, 2116, 1447, 1365, 1094, 1022, 914, 844, and $672 \mathrm{~cm}^{-1}$.

GC-MS $\left.50{ }^{\circ} \mathrm{C} / 2.0 \mathrm{~min} / 20^{\circ} \mathrm{C} \mathrm{min}-1 / 250{ }^{\circ} \mathrm{C}\right) t R=7.02 \mathrm{~min}$; $\mathrm{MS}\left[70 \mathrm{eV}, \mathrm{m} / z\right.$ (rel int)]: $142\left(1, \mathrm{C}_{7} \mathrm{H}_{10} \mathrm{O}_{3}{ }^{+}\right)$, $82\left(24, \mathrm{C}_{5} \mathrm{H}_{6} \mathrm{O}_{2}^{+}\right)$, and $69\left(100, \mathrm{C}_{4} \mathrm{H}_{5} \mathrm{O}^{+}\right.$, protonated furan).

HRMS (ESI) m/z: [M + Na]+ Calcd for $\mathrm{C}_{9} \mathrm{H}_{12} \mathrm{NaO}_{4}{ }^{+}$207.0628; Found 207.0620. 
(3R,3aR,6S,6aR)-6-(Prop-2-yn-1-yloxy)hexahydrofuro[3,2-b]furan-3-yl acetate (3)

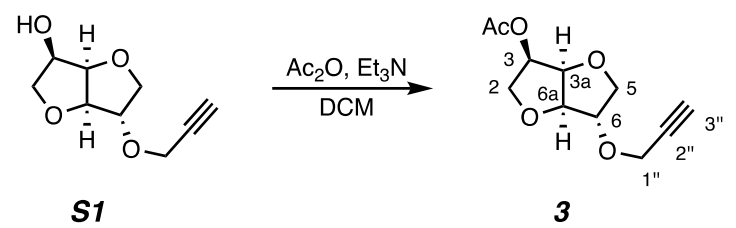

The exo-propargyl ether $\mathbf{S 1}$ (150 $\mathrm{mg}, 0.82 \mathrm{mmol}$ ) was dissolved in $5 \mathrm{~mL}$ of dichloromethane in a culture tube equipped with a stir bar. Acetic anhydride $(205 \mu \mathrm{L}, 3.3 \mathrm{mmol})$ and triethylamine $(303 \mu \mathrm{L}, 3.3 \mathrm{mmol})$ were added sequentially via a Wiretrol ${ }^{\mathrm{TM}}$. The tube was resealed and allowed to stir at ambient temperature overnight. The reaction mixture was concentrated in vacuo and filtered through plugs of silica and basic alumina (1:1 hexanes/EtOAc). The filtrate was concentrated in vacuo and acetate 3 was recovered as a paleyellow oil (180 mg, 98\%).

${ }^{1}$ H NMR $\left(500 \mathrm{MHz}, \mathrm{CDCl}_{3}\right): \delta 5.14(\mathrm{ddd}, J=6.0,6.0,5.2 \mathrm{~Hz}, 1 \mathrm{H}, H 3), 4.78(\mathrm{dd}, J=5.2,4.6, \mathrm{~Hz}, 1 \mathrm{H}$, $H 3 \mathrm{a}$ ), 4.53 (ddd, $J=4.6,1.0,1.0 \mathrm{~Hz}, 1 \mathrm{H}, H 6 \mathrm{a}), 4.24$ (dddd, $J=3.9,1.6,1.0,0.6 \mathrm{~Hz}, 1 \mathrm{H}, H 6), 4.22$ (dd, $J$ $\left.=16.1,2.4 \mathrm{~Hz}, 1 \mathrm{H}, \mathrm{C} 1^{\prime} H_{\mathrm{a}} \mathrm{H}_{\mathrm{b}}\right), 4.18\left(\mathrm{dd}, J=16.1,2.4 \mathrm{~Hz}, 1 \mathrm{H}, \mathrm{C} 1^{\prime} \mathrm{H}_{\mathrm{a}} H_{\mathrm{b}}\right), 4.02(\mathrm{ddd}, J=10.3,1.4,0.9 \mathrm{~Hz}$, $\left.1 \mathrm{H}, \mathrm{C} 5 \mathrm{H}_{\mathrm{a}} H_{\mathrm{b}}\right), 3.95\left(\mathrm{dd}, J=9.5,6.1 \mathrm{~Hz}, 1 \mathrm{H}, \mathrm{C} 2 H_{\mathrm{a}} \mathrm{H}_{\mathrm{b}}\right), 3.92\left(\mathrm{dd}, J=10.3,3.8 \mathrm{~Hz}, 1 \mathrm{H}, \mathrm{C} \mathrm{H}_{\mathrm{a}} H_{\mathrm{b}}\right), 3.75$ (ddd, $\left.J=9.5,5.9,0.6 \mathrm{~Hz}, 1 \mathrm{H}, \mathrm{C}_{2} \mathrm{H}_{\mathrm{a}} H_{\mathrm{b}}\right), 2.46\left(\mathrm{t}, J=2.4 \mathrm{~Hz}, 1 \mathrm{H}, \mathrm{H3}{ }^{\prime}\right)$, and $2.12\left(\mathrm{~s}, 3 \mathrm{H}, \mathrm{C} 3 \mathrm{COOCH}_{3}\right)$.

${ }^{13}$ C NMR (125 MHz, DMSO- $\left.d_{6}\right): \delta 170.4,85.9,82.8,80.6,79.1,75.1,74.0,73.0,69.9,56.8$, and 20.7. FT-IR (thin film): 3266, 2933, 2873, 1736, 1368, 1232, 1091, 1055, and $645 \mathrm{~cm}^{-1}$.

GC-MS $\left.50{ }^{\circ} \mathrm{C} / 2.0 \mathrm{~min} / 20{ }^{\circ} \mathrm{C} \mathrm{min}-1 / 250{ }^{\circ} \mathrm{C}\right) t R=7.76 \mathrm{~min}$; MS $\left[70 \mathrm{eV}, \mathrm{m} / z\right.$ (rel int)]: $226\left(1, \mathrm{M}^{+}\right), 166$ $\left(11 \mathrm{C}_{9} \mathrm{H}_{10} \mathrm{O}_{3}^{+}\right), 82\left(76, \mathrm{C}_{5} \mathrm{H}_{6} \mathrm{O}_{2}^{+}\right)$, and $69\left(100, \mathrm{C}_{4} \mathrm{H}_{5} \mathrm{O}^{+}\right.$, protonated furan).

HRMS (ESI) m/z: [M + H] $]^{+}$Calcd for $\mathrm{C}_{11} \mathrm{H}_{15} \mathrm{O}_{5}{ }^{+}$227.0919; Found 227.0907. 
$\left(3 R, 3 \mathrm{a} R, 3 ' R, 3 \mathrm{a}^{\prime} R, 6 S, 6 \mathrm{a} R, 6\right.$ ' $\left.S, 6 \mathrm{a}^{\prime} R\right)$-(Hexa-2,4-diyne-1,6-diylbis(oxy))bis(hexahydrofuro[3,2-b]furan6,3-diyl) Diacetate (4)

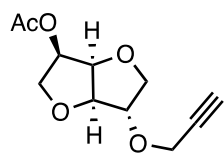

3

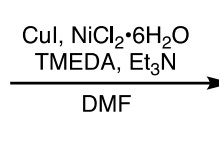

DMF

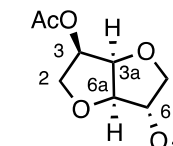

$\overline{\mathrm{H}}: \overline{\mathrm{O}}$

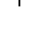

4
$88 \%$

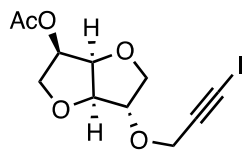

5

Cuprous iodide $(4.2 \mathrm{mg}, 0.02 \mathrm{mmol}$ ) was suspended in $2 \mathrm{~mL}$ of dimethylformamide in a 6-dram vial equipped with a micro stir bar. $N, N, N^{\prime}, N^{\prime}$-Tetramethylethylenediamine $(0.17 \mathrm{mmol}, 27 \mu \mathrm{L})$ was added to the vial under stirring, forming a deep blue homogenous solution, followed by addition of nickel (II) chloride hexahydrate $(5.3 \mathrm{mg}, 0.02 \mathrm{mmol})$, turning the solution to a deep green. Triethylamine $(0.19 \mathrm{~mL}$, $1.32 \mathrm{mmol}$ ) was added dropwise via syringe, and the vial was sealed with a rubber septum and allowed to stir for one hour. A solution of $3(100 \mathrm{mg}, 0.45 \mathrm{mmol})$ in $0.5 \mathrm{~mL}$ of DMF was injected into the vial, which was sealed once again with a septum. The headspace was purged with an oxygen balloon, and the reaction mixture was allowed to stir under oxygen at room temperature for $72 \mathrm{~h}$. The reaction mixture was poured into $10 \mathrm{~mL}$ of satd ammonium bicarbonate and extracted $3 \mathrm{x}$ with EtOAc. The combined organic layers were washed with DI water $(3 \mathrm{x})$ and brine $(2 \mathrm{x})$, dried $\left(\mathrm{MgSO}_{4}\right)$, and concentrated in vacuo. The residue was purified by MPLC (1:1 hexanes/EtOAc) to yield, in order of elution, the alkynyl iodide 5 (5 mg, 6\%), containing a similar amount of the coeluting alkyne $\mathbf{3}$, and the diyne $4(87 \mathrm{mg}, 88 \%)$ as a sticky oil.

\section{Characterization data for 4}

${ }^{1} \mathbf{H}$ NMR (500 MHz, $\left.\mathrm{CDCl}_{3}\right): \delta 5.13(\mathrm{ddd}, J=6.0,5.95 .3 \mathrm{~Hz}, 2 \mathrm{H}, H 3), 4.77$ (dd, $\left.J=5.3,4.6 \mathrm{~Hz}, 2 \mathrm{H}, H 3 \mathrm{a}\right)$, 4.50 (ddd, $J=4.6,1.0,1.0 \mathrm{~Hz}, 2 \mathrm{H}, H 6 \mathrm{a}), 4.28$ (br d, $J=16.8 \mathrm{~Hz}, 2 \mathrm{H}, \mathrm{C} 1{ }^{\prime} H_{\mathrm{a}} \mathrm{H}_{\mathrm{b}}$ ), 4.27 (br d, $J=16.9 \mathrm{~Hz}$, $2 \mathrm{H}, \mathrm{C} 1{ }^{\prime} \mathrm{H}_{\mathrm{a}} H_{\mathrm{b}}$ ), 4.20 (dddd, $\left.J=3.9,1.6,1.0,0.6 \mathrm{~Hz}, 2 \mathrm{H}, H 6\right), 4.00$ (ddd, $J=10.3,1.5,0.9 \mathrm{~Hz}, 2 \mathrm{H}, \mathrm{C}^{2} \mathrm{H}_{\mathrm{a}} H_{\mathrm{b}}$ ), $3.95\left(\mathrm{dd}, J=9.6,6.1 \mathrm{~Hz}, 2 \mathrm{H}, \mathrm{C} 2 H_{\mathrm{a}} \mathrm{H}_{\mathrm{b}}\right), 3.91\left(\mathrm{dd}, J=10.3,3.9 \mathrm{~Hz}, 2 \mathrm{H}, \mathrm{C} 5 \mathrm{H}_{\mathrm{a}} H_{\mathrm{b}}\right), 3.75(\mathrm{ddd}, J=9.6,5.8$, $\left.0.6 \mathrm{~Hz}, 2 \mathrm{H}, \mathrm{C} 2 \mathrm{H}_{\mathrm{a}} \mathrm{H}_{\mathrm{b}}\right)$, and $2.11\left(\mathrm{~s}, 6 \mathrm{H}, \mathrm{C} 3 \mathrm{COOCH}_{3}\right)$.

${ }^{13}$ C NMR (125 MHz, DMSO- $\left.d_{6}\right): \delta 170.4,85.8,83.1,80.6,75.0,74.0,72.9,70.7,70.0,57.2$, and 20.7.

FT-IR (thin film): 2932, 2874, 1738, 1367, 1232, 1091, 1055, and $644 \mathrm{~cm}^{-1}$.

HRMS (ESI) m/z: [M+ H] $]^{+}$Calcd for $\mathrm{C}_{22} \mathrm{H}_{27} \mathrm{O}_{10}{ }^{+} 451.1599$; Found 451.1586. 
(3R,3aR,6S,6aR)-6-((3-Iodoprop-2-yn-1-yl)oxy)hexahydrofuro[3,2-b]furan-3-yl Acetate (5)

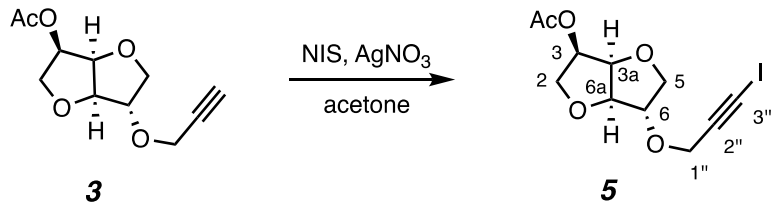

endo-Acetate 3 ( $25 \mathrm{mg}, 0.11 \mathrm{mmol}$ ) was dissolved in $2 \mathrm{~mL}$ of acetone in a threaded culture tube fitted with a stir bar. $N$-Iodosuccinimide $(30 \mathrm{mg}, 1.3 \mathrm{mmol})$ and silver nitrate $(1.9 \mathrm{mg}, 0.1 \mathrm{mmol})$ were added to the culture tube, which was sealed and allowed to stir for $15 \mathrm{~h}$ at ambient temperature. The reaction mixture was poured into DI water and extracted $3 \mathrm{x}$ with dichloromethane. The combined extracts were dried $\left(\mathrm{MgSO}_{4}\right)$ and concentrated in vacuo. The residue was filtered through a short plug of silica (1:1 hexanes/EtOAc) and the filtrate was concentrated in vacuo to afford iodoalkyne $\mathbf{5}$ (31 $\mathrm{mg}, 81 \%$ recovered).

${ }^{1} \mathbf{H}$ NMR $\left(500 \mathrm{MHz}, \mathrm{CDCl}_{3}\right): \delta 5.12(\mathrm{ddd}, J=6.0,5.9,5.2 \mathrm{~Hz}, 1 \mathrm{H}, H 3), 4.76(\mathrm{dd}, J=5.2,4.6,0.6 \mathrm{~Hz}, 1 \mathrm{H}$, $H 3 \mathrm{a}$ ), 4.48 (ddd, $J=4.6,1.0,1.0, \mathrm{~Hz}, 1 \mathrm{H}, H 6 \mathrm{a}), 4.35$ (d, $\left.J=16.0 \mathrm{~Hz}, 1 \mathrm{H}, \mathrm{C} 1{ }^{\prime} H_{\mathrm{a}} \mathrm{H}_{\mathrm{b}}\right), 4.34$ (d, $J=16.0 \mathrm{~Hz}$, $\left.1 \mathrm{H}, \mathrm{C} 1^{\prime} \mathrm{H}_{\mathrm{a}} H_{\mathrm{b}}\right), 4.21$ (dddd, $\left.J=3.9,1.6,1.0,0.6 \mathrm{~Hz}, 1 \mathrm{H}, H 6\right), 4.00$ (ddd, $J=10.3,1.6,1.0 \mathrm{~Hz}, 1 \mathrm{H}, \mathrm{C} \mathrm{H}_{\mathrm{a}} H_{\mathrm{b}}$ ), $3.94\left(\mathrm{dd}, J=9.6,6.1 \mathrm{~Hz}, 1 \mathrm{H}, \mathrm{C} 2 H_{\mathrm{a}} \mathrm{H}_{\mathrm{b}}\right), 3.90\left(\mathrm{dd}, J=10.3,3.9 \mathrm{~Hz}, 1 \mathrm{H}, \mathrm{C} 5 \mathrm{H}_{\mathrm{a}} H_{\mathrm{b}}\right), 3.75(\mathrm{ddd}, J=9.5,5.9$, $\left.0.6 \mathrm{~Hz}, 1 \mathrm{H}, \mathrm{C} 2 \mathrm{H}_{\mathrm{a}} \mathrm{H}_{\mathrm{b}}\right)$, and $2.11\left(\mathrm{~s}, 3 \mathrm{H}, \mathrm{C} 3 \mathrm{COOCH}_{3}\right)$.

${ }^{13} \mathrm{C}$ NMR $\left(125 \mathrm{MHz}, \mathrm{CDCl}_{3}\right): \delta 170.4,89.7,85.9,82.8,80.6,74.0,72.9,69.9,58.3,20.7$, and 3.9.

GC-MS $50{ }^{\circ} \mathrm{C} / 2.0 \mathrm{~min} / 20{ }^{\circ} \mathrm{C} \mathrm{min}-1 / 250{ }^{\circ} \mathrm{C}$ ) $t R=10.11 \mathrm{~min} ; \mathrm{MS}[70 \mathrm{eV}, \mathrm{m} / z$ (rel int)]: $310(1, \mathrm{M}+), 224$ $\left(38,-\mathrm{I}^{+}\right), 165\left(100,{ }^{+} \mathrm{CH}_{2} \mathrm{C} \equiv \mathrm{CI}\right)$, and $69\left(81, \mathrm{C}_{4} \mathrm{H}_{5} \mathrm{O}^{+}\right.$, protonated furan).

FT-IR (thin film): 2875, 1736, 1366, 1367, 1232, 1090, 1056, and $732 \mathrm{~cm}^{-1}$.

HRMS (ESI) m/z: [M + H] $]^{+}$Calcd for $\mathrm{C}_{11} \mathrm{H}_{16} \mathrm{IO}_{5}{ }^{+}$352.9886; Found 352.9865 . 
(3R,3aR,6S,6aR)-3,6-bis((3-Iodoprop-2-yn-1-yl)oxy)hexahydrofuro[3,2-b]furan (6)

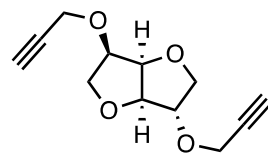

$2 a$

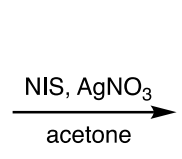

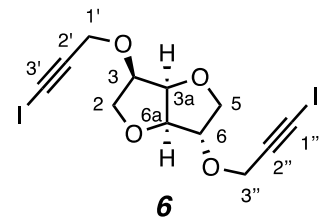

bis-Propargyl ether 2a (200 mg, $0.90 \mathrm{mmol})$ was dissolved in $2 \mathrm{~mL}$ of acetone in a screw-cap vial fitted with a stir bar. $N$-Iodosuccinimide $(507 \mathrm{mg}, 2.3 \mathrm{mmol})$ and silver nitrate $(30 \mathrm{mg}, 0.18 \mathrm{mmol})$ were added to the culture tube, which was sealed and allowed to stir for $15 \mathrm{~h}$ at ambient temperature. The reaction mixture was poured into DI water and extracted $3 \mathrm{x}$ with dichloromethane. The combined extracts were dried $\left(\mathrm{MgSO}_{4}\right)$ and concentrated in vacuo. The residue was filtered through a short plug of silica $(1: 1$ hexanes/EtOAc) and the filtrate was concentrated in vacuo to afford bis-iodoalkyne 6 ( $239 \mathrm{mg}, 56 \%$ yield).

${ }^{1}$ H NMR $\left(500 \mathrm{MHz}, \mathrm{DMSO}-d_{6}\right): \delta 4.55$ (dd, $\left.J=4.6,4.6 \mathrm{~Hz}, 1 \mathrm{H}, H 3 \mathrm{a}\right), 4.45$ (ddd, $J=4.6,1.1,1.1 \mathrm{~Hz}, 1 \mathrm{H}$, $H 6 \mathrm{a}), 4.39$ (d, $\left.J=15.9 \mathrm{~Hz}, 1 \mathrm{H}, \mathrm{C} 1^{\prime} H_{\mathrm{a}} \mathrm{H}_{\mathrm{b}}\right), 4.34$ (s, 2H, C1' ' $\left.H_{2}\right), 4.31$ (d, $\left.J=15.9 \mathrm{~Hz}, 1 \mathrm{H}, \mathrm{Cl}{ }^{\prime} \mathrm{H}_{\mathrm{a}} H_{\mathrm{b}}\right), 4.10$ (ddd, $J=7.2,6.4,4.7 \mathrm{~Hz}, 1 \mathrm{H}, H 3$ ), 4.02 (br d, $J=4.1 \mathrm{~Hz}, 1 \mathrm{H}, H 6), 3.86$ (ddd, $J=10.3,1.2,1.2 \mathrm{~Hz}, 1 \mathrm{H}$, $\left.\mathrm{C} 5 H_{\mathrm{a}} \mathrm{H}_{\mathrm{b}}\right), 3.82\left(\mathrm{dd}, J=8.8,6.5 \mathrm{~Hz}, 1 \mathrm{H}, \mathrm{C} 2 \mathrm{H}_{\mathrm{a}} \mathrm{H}_{\mathrm{b}}\right), 3.75\left(\mathrm{dd}, J=10.3,3.8 \mathrm{~Hz}, 1 \mathrm{H}, \mathrm{C} \mathrm{H}_{\mathrm{a}} H_{\mathrm{b}}\right)$, and $3.45(\mathrm{dd}, J$ $\left.=8.8,7.2 \mathrm{~Hz}, 1 \mathrm{H}, \mathrm{C} 2 \mathrm{H}_{\mathrm{a}} H_{\mathrm{b}}\right)$.

${ }^{13}$ C NMR (125 MHz, DMSO- $\left.d_{6}\right): \delta 89.8,85.6,83.0,80.2,78.9,72.9,72.8,69.9,58.9,58.1,13.7$, and 13.6.

FT-IR (thin film): 2931, 2872, 2182, 1077, 1059, and $832 \mathrm{~cm}^{-1}$.

GC-MS $\left.50{ }^{\circ} \mathrm{C} / 2.0 \mathrm{~min} / 20^{\circ} \mathrm{C} \mathrm{min}-1 / 250{ }^{\circ} \mathrm{C}\right) t R=12.33 \mathrm{~min} ; \mathrm{MS}[70 \mathrm{eV}, \mathrm{m} / z$ (rel int)]: $165(100$, $\left.{ }^{+} \mathrm{CH}_{2} \mathrm{C} \equiv \mathrm{CI}\right), 127\left(30, \mathrm{I}^{+}\right)$, and $69\left(40, \mathrm{C}_{4} \mathrm{H}_{5} \mathrm{O}^{+}\right.$, protonated furan).

HRMS (ESI) m/z: [M + Na $]^{+}$Calcd for $\mathrm{C}_{12} \mathrm{H}_{12} \mathrm{I}_{2} \mathrm{NaO}_{4}{ }^{+} 496.8717$; Found 496.8696 . 


\section{$(3 R, 3 a R, 6 S, 6 a R)-3,6-D i p r o p o x y h e x a h y d r o f u r o[3,2-b]$ furan (7)}

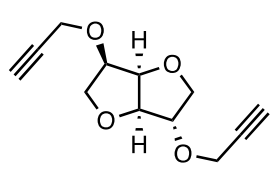

$2 a$

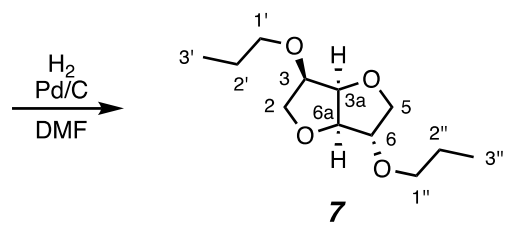

Preparation of 7: To a solution of diyne 2a $(100 \mathrm{mg}, 0.43 \mathrm{mmol})$ dissolved in $1 \mathrm{~mL}$ of $N, N-$ dimethylformamide was added $\mathrm{Pd} / \mathrm{C}(10 \mathrm{wt} \%$, ca. $10 \mathrm{mg})$ inside an open 6 dram vial. The vial was placed into a $100 \mathrm{~mL}$ Fischer-Porter vessel, which was then sealed and twice pressurized with hydrogen gas and vented. The vessel was again pressurized to $80 \mathrm{psi}$, and the black suspension was magnetically stirred for $24 \mathrm{~h}$ at ambient temperature. The pressure was vented, and the suspension was filtered through a pad of Celite $^{\circledR}$. The filtrate was concentrated in vacuo to afford $91 \mathrm{mg}$ of the bis-propyl ether 7 in $92 \%$ isolated yield.
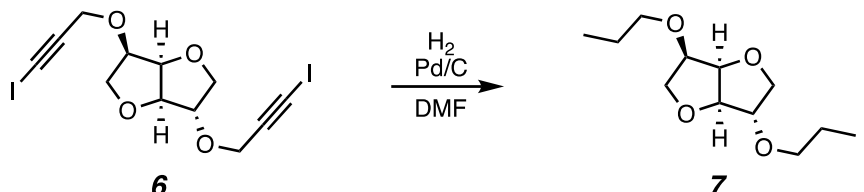

Hydrogenation of 6 to 7: To a solution of diyne $6(50 \mathrm{mg}, 0.11 \mathrm{mmol})$ dissolved in $1 \mathrm{~mL}$ of $N, N$ dimethylformamide was added $\mathrm{Pd} / \mathrm{C}(10 \mathrm{wt} \%$, ca. $10 \mathrm{mg})$ inside an open 6 dram vial. A portion of solid sodium bicarbonate (ca. $20 \mathrm{mg}$ ) was also added to the suspension. The vial was placed into a $100 \mathrm{~mL}$ Fischer-Porter vessel, which was then sealed and twice pressurized with hydrogen gas and vented. The vessel was again pressurized to $80 \mathrm{psi}$, and the black suspension was magnetically stirred for $24 \mathrm{~h}$ at ambient temperature. The pressure was vented and the suspension was filtered through a pad of Celite ${ }^{\circledR}$. The filtrate was concentrated in vacuo to afford $21 \mathrm{mg}$ of the bis-propyl ether 7 in $92 \%$ isolated yield. This material was judged to be substantially identical to that made from 2a by ${ }^{1} \mathrm{H}$ NMR and GC-MS analyses.

\section{Characterization Data for 7:}

${ }^{1}$ H NMR $\left(500 \mathrm{MHz}, \mathrm{DMSO}-d_{6}\right): \delta 4.53(\mathrm{dd}, J=4.6,4.6 \mathrm{~Hz}, 1 \mathrm{H}, H 3 \mathrm{a}), 4.40$ (ddd, $J=4.4,0.8,0.8 \mathrm{~Hz}, 1 \mathrm{H}$, $H 6 \mathrm{a}$ ), 3.94 (ddd, $J=7.7,6.6,4.6 \mathrm{~Hz}, 1 \mathrm{H}, H 3$ ), 3.86 (nfom, $1 \mathrm{H}, H 6$ ), 3.79 (dd, $J=8.4,6.5 \mathrm{~Hz}, 1 \mathrm{H}, \mathrm{C} 2 \mathrm{H}_{\mathrm{a}} \mathrm{H}_{\mathrm{b}}$ ), 3.81-3.77 (m, $\left.1 \mathrm{H}, \mathrm{C} 5 H_{\mathrm{a}} \mathrm{H}_{\mathrm{b}}\right), 3.76\left(\mathrm{dd}, J=9.9,3.9 \mathrm{~Hz}, 1 \mathrm{H}, \mathrm{C} 5 \mathrm{H}_{\mathrm{a}} H_{\mathrm{b}}\right), 3.52(\mathrm{ddd}, J=9.1,6.7,6.7 \mathrm{~Hz}, 1 \mathrm{H}$, $\mathrm{OCH}_{\mathrm{a}} \mathrm{H}_{\mathrm{b}} \mathrm{Et}$ ), 3.40 (ddd, $J=9.1,6.6,6.6 \mathrm{~Hz}, 1 \mathrm{H}, \mathrm{OCH}_{\mathrm{a}} \mathrm{H}_{\mathrm{b}} \mathrm{Et}$ '), $3.39\left(\mathrm{~m}, 1 \mathrm{H}, \mathrm{C} \mathrm{H}_{\mathrm{a}} H_{\mathrm{b}}\right.$ ), 3.36 (ddd, $J=9.1$, 6.6, $6.6 \mathrm{~Hz}, 1 \mathrm{H}, \mathrm{OCH}_{\mathrm{a}} H_{\mathrm{b}} \mathrm{Et}$ ') 3.35 (ddd, $J=9.2,6.6,6.6 \mathrm{~Hz}, 1 \mathrm{H}, \mathrm{OCH}_{\mathrm{a}} H_{\mathrm{b}} \mathrm{Et}$ ), 1.50 (overlapping m, $4 \mathrm{H}$, $\mathrm{OCH}_{2} \mathrm{CH}_{2} \mathrm{Me}$ and $\mathrm{OCH}_{2} \mathrm{CH}_{2} \mathrm{Me}$ '), 0.860 (t, $\left.J=7.4 \mathrm{~Hz}, 3 \mathrm{H}, \mathrm{O}\left(\mathrm{CH}_{2}\right)_{2} \mathrm{C}^{\prime} H_{3}\right)$, and $0.856(\mathrm{t}, J=7.4 \mathrm{~Hz}, 3 \mathrm{H}$, $\left.\mathrm{O}\left(\mathrm{CH}_{2}\right)_{2} \mathrm{C} 3 \mathrm{H}_{3}\right)$.

${ }^{13}$ C NMR (125 MHz, DMSO- $\left.d_{6}\right): \delta 86.0,84.2,80.2,80.1,73.0,71.6,70.7,69.8,23.1,23.0$, and 11.0(2x).

FT-IR 2961, 2935, 2874, 1447, 1370, 1093, 1074, and $1015 \mathrm{~cm}^{-1}$.

GC-MS $\left.50{ }^{\circ} \mathrm{C} / 2.0 \mathrm{~min} / 20^{\circ} \mathrm{C} \mathrm{min}-1 / 250{ }^{\circ} \mathrm{C}\right) t R=7.55 \mathrm{~min}$; MS [70 eV, $\mathrm{m} / z$ (rel int)]: $230(19, \mathrm{M}+), 127$ $\left(39, \mathrm{C}_{7} \mathrm{H}_{11} \mathrm{O}_{2}^{+}\right), 103\left(39, \mathrm{M} \mathrm{C}_{5} \mathrm{H}_{11} \mathrm{O}_{2}^{+}\right), 86\left(25, \mathrm{C}_{5} \mathrm{H}_{10} \mathrm{O}^{+}\right)$, and $69\left(100, \mathrm{C}_{4} \mathrm{H}_{5} \mathrm{O}^{+}\right.$, protonated furan).

HRMS (ESI) m/z: [M+ H] ${ }^{+}$Calcd for $\mathrm{C}_{12} \mathrm{H}_{23} \mathrm{O}_{4}{ }^{+}$231.1591; Found 231.1583. 
$\left(3 R, 3 \mathrm{a} R, 3 ' R, 3 \mathrm{a}^{\prime} R, 6 S, 6 \mathrm{a} R, 6\right.$ ' $\left.S, 6 \mathrm{a}^{\prime} R\right)$-6,6'-(Hexa-2,4-diyne-1,6-diylbis(oxy))bis(hexahydro-furo[3,2b]furan-3-ol) (S3)

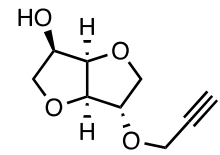

S1
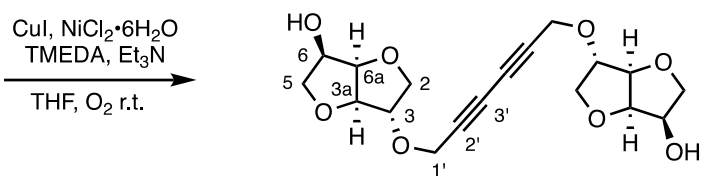

S3

Cuprous iodide (51 mg, $0.27 \mathrm{mmol}$ ) was suspended in $3 \mathrm{~mL}$ of tetrahydrofuran in a round-bottom flask equipped with a stir bar. $N, N, N^{\prime}, N^{\prime}$-Tetramethylethylenediamine $(250 \mu \mathrm{L}, 1.7 \mathrm{mmol})$ was added to the vial under stirring, forming a light green homogenous solution, followed by addition of nickel (II) chloride hexahydrate $(65 \mathrm{mg}, 0.27 \mathrm{mmol})$, turning the solution to a deep green. Triethylamine $(1.0 \mathrm{~mL}, 1.4 \mathrm{mmol})$ was added dropwise via syringe, and the vial was sealed with a rubber septum and allowed to stir for one hour. A solution of $\mathbf{S 1}(991 \mathrm{mg}, 5.4 \mathrm{mmol})$ in $0.5 \mathrm{~mL}$ of THF was injected into the vial, which was sealed once again with a septum. The solution and headspace were gently sparged with oxygen (balloon) fitted with a long needle over the course of ca. one hour, and the reaction mixture was stirred at room temperature for $48 \mathrm{~h}$. The crude reaction mixture was filtered through a short plug of silica (100\% EtOAc as the eluent). The filtrate was concentrated in vacuo and purified by MPLC (100\% EtOAc) to afford the exo/exo-diyne S3 (845 $\mathrm{mg}, 86 \%)$ as a yellow viscous oil.

${ }^{1}$ H NMR (500 MHz, DMSO- $d_{6}$ ): $\delta 4.83$ (dd, $\left.J=6.5,0.6 \mathrm{~Hz}, 2 \mathrm{H}, \mathrm{C} 6 \mathrm{OH}\right), 4.44$ (ddd, $J=4.4,1.1,1.1 \mathrm{~Hz}$, 2H, H3a), 4.38 (s, 4H, C1' $H_{2}$ ), 4.34 (dd, $\left.J=4.7,4.7 \mathrm{~Hz}, 2 \mathrm{H}, H 6 \mathrm{a}\right), 4.10$ (dddd, $J=7.8,6.4,6.4,4.8 \mathrm{~Hz}$, 2H, H6), 4.05 (dddd, $J=3.8,1.9,1.4,0.6 \mathrm{~Hz}, 2 \mathrm{H}, H 3$ ), 3.88 (ddd, $J=10.3,1.3,1.3, \mathrm{~Hz}, 2 \mathrm{H}, \mathrm{C} 2 H_{\mathrm{a}} \mathrm{H}_{\mathrm{b}}$ ), 3.77 $\left(\mathrm{dd}, J=10.3,3.8 \mathrm{~Hz}, 2 \mathrm{H}, \mathrm{C} 2 \mathrm{H}_{\mathrm{a}} H_{b}\right), 3.72\left(\mathrm{dd}, J=8.4,6.5 \mathrm{~Hz}, 2 \mathrm{H}, \mathrm{C} 5 H_{a} \mathrm{H}_{\mathrm{b}}\right)$, and 3.30 (ddd, $J=8.3,7.7,0.6$ $\left.\mathrm{Hz}, 2 \mathrm{H}, \mathrm{C} 5 \mathrm{H}_{\mathrm{a}} H_{\mathrm{b}}\right)$.

${ }^{13}$ C NMR (125 MHz, DMSO- $\left.d_{6}\right): \delta 84.9,83.5,81.6,76.7,72.3,72.1,71.2,69.6$, and 56.6.

FT-IR (thin film): 3421 (v br), 2933, 2872, 1657, 1387, 1046, 914, and $661 \mathrm{~cm}^{-1}$.

HRMS (ESI) m/z: [M+ H] $]^{+}$Calcd for $\mathrm{C}_{18} \mathrm{H}_{23} \mathrm{O}_{8}{ }^{+} 367.1387$; Found 367.1374. 


\section{1,6-bis(((3S,3aR,6R,6aR)-6-(Prop-2-yn-1yloxy)hexahydrofuro[3,2-b]furan-3-yl)oxy)hexa-2,4-diyne} $\left(\mathbf{2 a}-\mathbf{R} \mathbf{R}^{*}\right)$

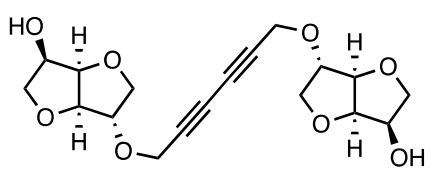

S3
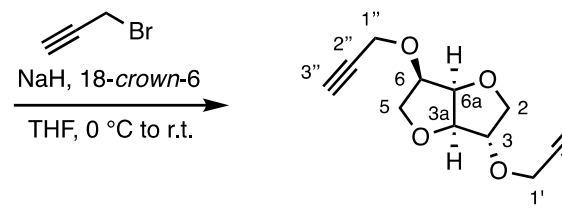

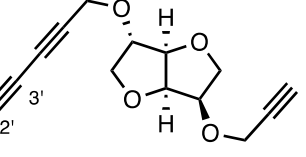

8

To a solution of crude $\mathbf{S 3}$ (600 mg, $1.6 \mathrm{mmol}$ ) in $10 \mathrm{~mL}$ of tetrahydrofuran was added 18-crown-6 (22 mg, $0.08 \mathrm{mmol}$ ) and a $60 \mathrm{wt} . \%$ dispersion in mineral oil of sodium hydride (180 $\mathrm{mg}, 4.3 \mathrm{mmol})$. The reaction mixture was concentrated, dissolved in DCM, and filtered through a plug of silica $(100 \%$ EtOAc as the eluent), and the resulting filtrate was concentrated to afford a brown residue. This was purified by MPLC (2:1 hexanes/EtOAc) to afford the $\alpha, \omega$-propargylated exo/exo-diyne $\mathbf{2 a - R R}$ as a light-yellow oil (442 mg, $61 \%$ yield).

${ }^{1}$ H NMR $\left(500 \mathrm{MHz}, \mathrm{DMSO}-d_{6}\right): \delta 4.58$ (dd, $\left.J=4.6,4.6 \mathrm{~Hz}, 2 \mathrm{H}, H 6 \mathrm{a}\right), 4.48$ (ddd, $J=4.5,1.0,1.0 \mathrm{~Hz}, 2 \mathrm{H}$, $H 3 \mathrm{a}), 4.39$ (s, 4H, C1' $\left.H_{2}\right), 4.27$ (dd, $\left.J=15.8,2.4 \mathrm{~Hz}, 2 \mathrm{H}, \mathrm{C} 1{ }^{\prime}{ }^{\prime} H_{\mathrm{a}} \mathrm{H}_{\mathrm{b}}\right), 4.18$ (dd, $J=15.8,2.4 \mathrm{~Hz}, 2 \mathrm{H}$, C1' ' $\mathrm{H}_{\mathrm{a}} H_{\mathrm{b}}$ ), 4.14 (ddd, $J=7.3,6.3,4.7 \mathrm{~Hz}, 2 \mathrm{H}, H 6$ ), 4.06 (dddd, $J=3.7,1.2,1.2 .0 .6 \mathrm{~Hz}, 2 \mathrm{H}, H 3$ ), 3.88 (ddd, $\left.J=10.3,1.1,1.1 \mathrm{~Hz}, 2 \mathrm{H}, \mathrm{C} 2 H_{\mathrm{a}} \mathrm{H}_{\mathrm{b}}\right), 3.82\left(\mathrm{dd}, J=8.8,6.4 \mathrm{~Hz}, 2 \mathrm{H}, \mathrm{C} 5 H_{\mathrm{a}} \mathrm{H}_{\mathrm{b}}\right), 3.75(\mathrm{dd}, J=10.3,3.7$ $\mathrm{Hz}, 2 \mathrm{H}, \mathrm{C} 2 \mathrm{H}_{\mathrm{a}} H_{\mathrm{b}}$ ), 3.47 (t, $J=2.4 \mathrm{~Hz}, 2 \mathrm{H}, H 3$ '), and 3.45 (ddd, $J=8.8,7.2 \mathrm{~Hz}, 0.6 \mathrm{~Hz} 2 \mathrm{H}, \mathrm{C} \mathrm{H}_{\mathrm{a}} H_{\mathrm{b}}$ ).

${ }^{13}$ C NMR (125 MHz, DMSO-d6): $\delta$ 85.6, 83.5, 80.7, 80.3, 78.9, 77.9, 77.0, 72.8, 70.0, 69.9, 57.3, and 57.0. FT-IR (thin film): 3269, 2923, 2871, 1449, 1362, 1127, 1060, 1020, and $664 \mathrm{~cm}^{-1}$.

HRMS (ESI) m/z: [M + H] $]^{+}$Calcd for $\mathrm{C}_{24} \mathrm{H}_{27} \mathrm{O}_{8}{ }^{+}$443.1700; Found 443.1691; [M + Na ${ }^{+}$Calcd for $\mathrm{C}_{24} \mathrm{H}_{26} \mathrm{NaO}_{8}{ }^{+}$465.1520; Found 465.1508.

* the descriptor "RR" in 2a-RR [and poly(2a-RR)] designates that this is a regioregular monomer (and polymer 
$(3 R, 3 a R, 6 S, 6 a R)-6-(F o r m y l o x y) h e x a h y d r o f u r o[3,2-b]$ furan-3-yl acetate (S5)

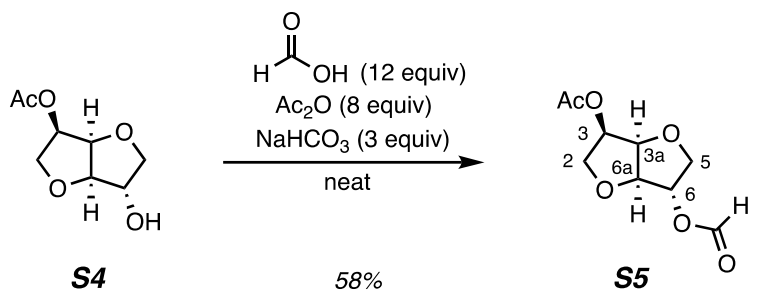

Formic acid (580 $\mu \mathrm{L}, 9.4 \mathrm{mmol}, 88 \%$ aq soln $)$ and acetic anhydride $(864 \mu \mathrm{L}, 6.2 \mathrm{mmol})$ were added to a threaded culture tube fitted with a stir bar and PTFE-lined cap were heated at $60{ }^{\circ} \mathrm{C}$ for 90 minutes. The solution of mixed anhydride was cooled to $0{ }^{\circ} \mathrm{C}$ in an ice water bath, and then added via pipet to the neat endo-acetate $\mathbf{S} 4^{4, \dagger}$ (146 mg, $0.78 \mathrm{mmol}$ ) in a separate vial, followed by addition of solid sodium bicarbonate (197 mg, $2.3 \mathrm{mmol}$ ). This mixture was allowed to warm to ambient temperature and stirred overnight, at which point the reaction mixture was quenched by dropwise addition to a solution of aq sodium bicarbonate. This was extracted 3x with dichloromethane. The combined organic layers were washed with brine, dried with anhyd $\mathrm{MgSO}_{4}$, and concentrated in vacuo to afford the formate ester $\mathbf{S 5}$ (98 $\mathrm{mg}, 58 \%$ recovered), which gave the following data without further purification.

${ }^{1}$ H NMR (500 MHz, $\mathrm{CDCl}_{3}$ ): $\delta 8.04(\mathrm{ddd}, J=0.9,0.9,0.9 \mathrm{~Hz}, 1 \mathrm{H}, \mathrm{C} 6 \mathrm{CO} H), 5.33-5.31$ (nfom, 1H, H6), 5.16 ( ddd, $J=6.1,5.3,5.3 \mathrm{~Hz}, 1 \mathrm{H}, H 3), 4.85$ (dd, $J=5.3,4.7 \mathrm{~Hz}, 1 \mathrm{H}, H 3 \mathrm{a}), 4.52$ (ddd, $J=4.6,0.8,0.8 \mathrm{~Hz}, 1 \mathrm{H}$, $H 6 \mathrm{a}), 4.03$ (ddd, $\left.J=10.8,3.0,0.9 \mathrm{~Hz}, 1 \mathrm{H}, \mathrm{C} 5 H_{\mathrm{a}} \mathrm{H}_{\mathrm{b}}\right), 4.01$ (dd, $J=10.8,1.8 \mathrm{~Hz}, 1 \mathrm{H}, \mathrm{C} 5 \mathrm{H}_{\mathrm{a}} H_{\mathrm{b}}$ ), 3.96 (dd, $J$ $\left.=9.8,6.0 \mathrm{~Hz}, 1 \mathrm{H}, \mathrm{C} 2 \mathrm{H}_{\mathrm{a}} \mathrm{H}_{\mathrm{b}}\right), 3.81\left(\mathrm{ddd}, J=9.8,5.5,0.6 \mathrm{~Hz}, 1 \mathrm{H}, \mathrm{C}_{2} \mathrm{H}_{\mathrm{a}} H_{\mathrm{b}}\right)$, and $2.12(\mathrm{~s}, 3 \mathrm{H}, \mathrm{C} 3 \mathrm{COOCH})_{3}$.

${ }^{13}$ C NMR (125 MHz, $\left.\mathrm{CDCl}_{3}\right): \delta 170.3,159.8,85.7,80.8,77.6,73.9,73.3,70.4$, and 20.7.

FT-IR (thin film): 2882, 2875, 1731, 1714, 1370, 1233, 1165, 1090, and $849 \mathrm{~cm}^{-1}$.

GC-MS $\left.50{ }^{\circ} \mathrm{C} / 2.0 \mathrm{~min} / 20{ }^{\circ} \mathrm{C} \mathrm{min}^{-1} / 250{ }^{\circ} \mathrm{C}\right) t R=7.10 \mathrm{~min}$; MS $\left[70 \mathrm{eV}, \mathrm{m} / z\right.$ (rel int)]: $170\left(5, \mathrm{M}-\mathrm{HCO}_{2} \mathrm{H}\right)$, $156\left(10, \mathrm{M}-\mathrm{H}_{3} \mathrm{CCO}_{2} \mathrm{H}\right), 110\left(98, \mathrm{C}_{6} \mathrm{H}_{6} \mathrm{O}_{2}{ }^{+}\right)$, and $69\left(64, \mathrm{C}_{4} \mathrm{H}_{5} \mathrm{O}^{+}\right.$, protonated furan).

HRMS (ESI) m/z: [M + H] $]^{+}$Calcd for $\mathrm{C}_{9} \mathrm{H}_{13} \mathrm{O}_{6}{ }^{+}$217.0712; Found 217.0697.

mp: $42-44^{\circ} \mathrm{C}$.

\footnotetext{
${ }^{\dagger}$ Kindly provided by Dr. Derek Saxon.
} 


\section{Preparation and Characterization of Polymers}

Typical procedure for the Glaser oxidative polymerization of isohexide bis-propargyl ethers 2a-c. Cuprous iodide (56 mg, $0.30 \mathrm{mmol}$ ) was suspended in $2 \mathrm{~mL}$ of dimethylformamide in a 6-dram vial equipped with a micro stir bar. $N, N, N^{\prime}, N^{\prime}$-Tetramethylethylenediamine $(2.4 \mathrm{mmol}, 360 \mu \mathrm{L})$ was added to the vial under stirring, forming a deep blue homogenous solution. Nickel (II) chloride hexahydrate (71 $\mathrm{mg}$, $0.30 \mathrm{mmol})$ was added, and the solution turned to a deep green. Triethylamine $(1.0 \mathrm{~mL}, 1.32 \mathrm{mmol})$ was added dropwise via syringe, and the vial was sealed with a rubber septum and allowed to stir for one hour. Diyne 2a (1.3 g, $0.45 \mathrm{mmol})$ was injected into the vial, which was sealed once again with a septum. The solution and headspace were sparged with an oxygen balloon fitted with a long needle, and the reaction mixture was allowed to stir under oxygen at room temperature for $48 \mathrm{~h}$. The polymer was precipitated by dropwise addition of the reaction mixture into $100 \mathrm{~mL}$ of a stirred satd ammonium chloride solution at ambient temperature. The resulting suspension was allowed to settle, and the supernatant liquid removed by decantation. The residual solid was washed with DI water $(2 \times 10 \mathrm{~mL})$. The vial was wrapped in foil, and the remaining wet solids were frozen in liquid nitrogen and the remaining water was removed on a lyophilizer. The poly(2a) was obtained as a fluffy, off-white powder (936 mg, 72\% yield).

$\operatorname{Poly}(2 a)$

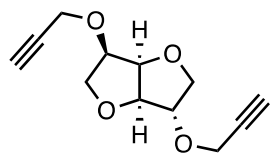

$2 a$

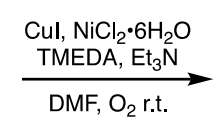

DMF, $\mathrm{O}_{2}$ r.t.

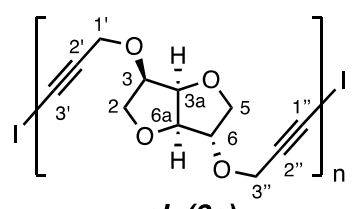

poly(2a)

${ }^{1}$ H NMR (500 MHz, DMSO- $d_{6}$ ): $\delta 4.60$ (dd, $\left.J=4.7,4.7 \mathrm{~Hz}, 1 \mathrm{H}, H 3 \mathrm{a}\right), 4.48$ (brd, $\left.J=4.7 \mathrm{~Hz}, 1 \mathrm{H}, H 6 \mathrm{a}\right)$, $4.43\left(\mathrm{~d} J=16.6 \mathrm{~Hz}, 1 \mathrm{H}, \mathrm{Cl}{ }^{\prime} H_{\mathrm{a}} \mathrm{H}_{\mathrm{b}}\right), 4.40\left(\mathrm{~s}, 1 \mathrm{H}, \mathrm{C} 1^{\prime} H_{2}\right), 4.36\left(\mathrm{~d}, J=16.6 \mathrm{~Hz}, 1 \mathrm{H}, \mathrm{C} 1{ }^{\prime} \mathrm{H}_{\mathrm{a}} H_{\mathrm{b}}\right), 4.14$ (ddd, $J$ $=6.7,4.7,4.7 \mathrm{~Hz}, 1 \mathrm{H}, H 3), 4.06(\mathrm{brd}, J=3.7 \mathrm{~Hz}, 1 \mathrm{H}, H 6), 3.89$ (brd, $\left.J=10.3 \mathrm{~Hz}, 1 \mathrm{H}, \mathrm{C} 5 H_{\mathrm{a}} \mathrm{H}_{\mathrm{b}}\right), 3.82$ $\left(\mathrm{dd}, J=8.9,6.4 \mathrm{~Hz}, 1 \mathrm{H}, \mathrm{C} 2 \mathrm{H}_{\mathrm{a}} \mathrm{H}_{\mathrm{b}}\right), 3.76\left(\mathrm{dd}, J=10.3,3.7 \mathrm{~Hz}, 1 \mathrm{H}, \mathrm{C} 5 \mathrm{H}_{\mathrm{a}} H_{\mathrm{b}}\right)$, and $3.46(\mathrm{dd}, J=8.9,6.5 \mathrm{~Hz}$, $\left.1 \mathrm{H}, \mathrm{C} 2 \mathrm{H}_{\mathrm{a}} H_{\mathrm{b}}\right)$.

${ }^{13}$ C NMR (125 MHz, DMSO- $d_{6}$ ): $\delta 85.6,83.4,80.3,79.3,77.2-76.9$ (ca. 4 br singlets, C2' and C2''), 72.8, 70.06(C1' or $\mathrm{C} 1$ '’), 70.02, 69.98(C1' or $\left.\mathrm{C} 1{ }^{\prime \prime}\right), 57.8$, and 57.0.

SEC-MALS $M_{\mathrm{n}}=12.5 \mathrm{kDa}, \emptyset=1.3$.

DSC $T_{\mathrm{g}}=55^{\circ} \mathrm{C}$.

TGA $T_{\mathrm{d} 5 \%}=253{ }^{\circ} \mathrm{C}$. 
$\operatorname{Poly}(2 b)$

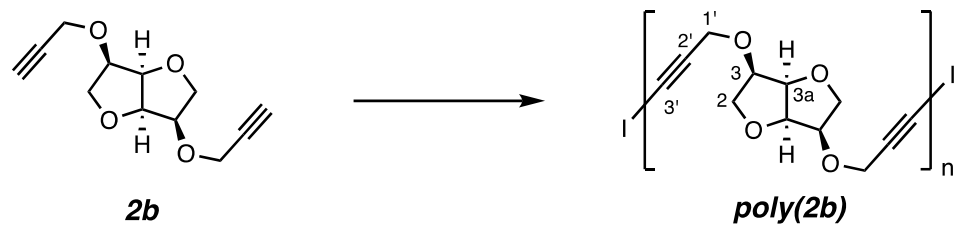

Poly(2b) was recovered as a fluffy, off-white powder (176 mg from $200 \mathrm{mg}$ of $\mathbf{2 c} ; 87 \%$ yield).

${ }^{1}$ H NMR (500 MHz, DMSO- $d_{6}$ ): $\delta 4.54$ (nfom, 2H, H3a), 4.42 (nfod, $J_{a p p}=16.2 \mathrm{~Hz}, 2 \mathrm{H}, \mathrm{Cl}^{\prime} H_{a} \mathrm{H}_{\mathrm{b}}$ ), 4.34 (nfod, $J_{a p p}=16.0 \mathrm{~Hz}, 2 \mathrm{H}, \mathrm{Cl}$ ' $\mathrm{H}_{\mathrm{a}} H_{b}$ ), 4.14 (dddd, $J=8.2,6.8,3.6,1.6 \mathrm{~Hz}, 2 \mathrm{H}, H 3$ ), 3.91 (dd, $J=8.4,6.9$ $\left.\mathrm{Hz}, 2 \mathrm{H}, \mathrm{C} 2 \mathrm{H}_{\mathrm{a}} \mathrm{H}_{\mathrm{b}}\right)$, and $3.46\left(\mathrm{dd}, J=8.3,8.3 \mathrm{~Hz}, 2 \mathrm{H}, \mathrm{C}_{2} \mathrm{H}_{\mathrm{a}} H_{b}\right)$.

${ }^{13}$ C NMR $\left(125 \mathrm{MHz}, \mathrm{DMSO}-d_{6}\right): \delta 80.3,79.3,77.1\left(\mathrm{C}_{\text {alkyne }}\right), 70.7,70.1\left(\mathrm{C}_{\text {alkyne }}\right)$, and $57.8\left(\mathrm{CH}_{2}\right)$.

SEC-MALS $M_{\mathrm{n}}=9.1 \mathrm{kDa}, \emptyset=1.5$.

DSC $T_{\mathrm{g}}=64^{\circ} \mathrm{C}$.

TGA $T_{\mathrm{d} 5 \%}=171^{\circ} \mathrm{C}$.

$\operatorname{Poly}(2 \mathrm{c})$

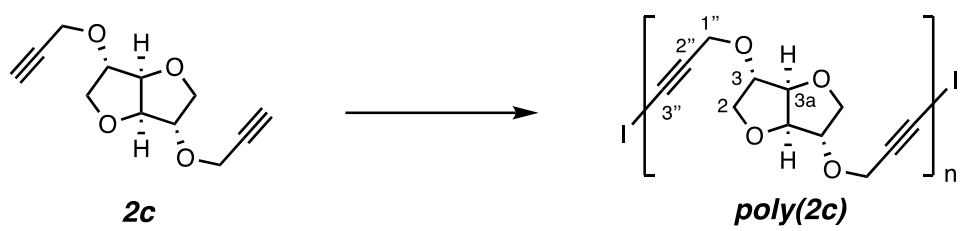

Poly(2c) was recovered as a fluffy, off-white powder (164 mg from $200 \mathrm{mg}$ of $\mathbf{2 c} ; 82 \%$ yield).

${ }^{1}$ H NMR (500 MHz, DMSO- $d_{6}$ ): $\delta 4.52$ (nfom, 2H, H3a), 4.41 (s, 4H, C1 '’ $H_{2}$ ), 4.07 (dd, $J=3.8,1.3 \mathrm{~Hz}$, $2 \mathrm{H}, H 3), 3.80\left(\mathrm{dd}, J=10.3,1.4 \mathrm{~Hz}, 2 \mathrm{H}, \mathrm{C} 2 H_{\mathrm{a}} \mathrm{H}_{\mathrm{b}}\right)$, and $3.46\left(\mathrm{dd}, J=10.3,3.8 \mathrm{~Hz}, 2 \mathrm{H}, \mathrm{C}_{2} \mathrm{H}_{\mathrm{a}} H_{b}\right)$.

${ }^{13}$ C NMR (125 MHz, DMSO- $\left.d_{6}\right): \delta 84.9,82.9,77.0\left(\mathrm{C}_{\text {alkyne }}\right), 71.7,70.0\left(\mathrm{C}_{\text {alkyne }}\right)$, and 57.1 $\left(\mathrm{CH}_{2}\right)$.

SEC-MALS $M_{\mathrm{n}}=8.9 \mathrm{kDa}, \emptyset=1.5$.

DSC $T_{\mathrm{g}}=56^{\circ} \mathrm{C}$.

TGA $T_{\mathrm{d} 5 \%}=178^{\circ} \mathrm{C}$. 


\section{$\operatorname{Poly}(2 a-R R)^{\ddagger}$}
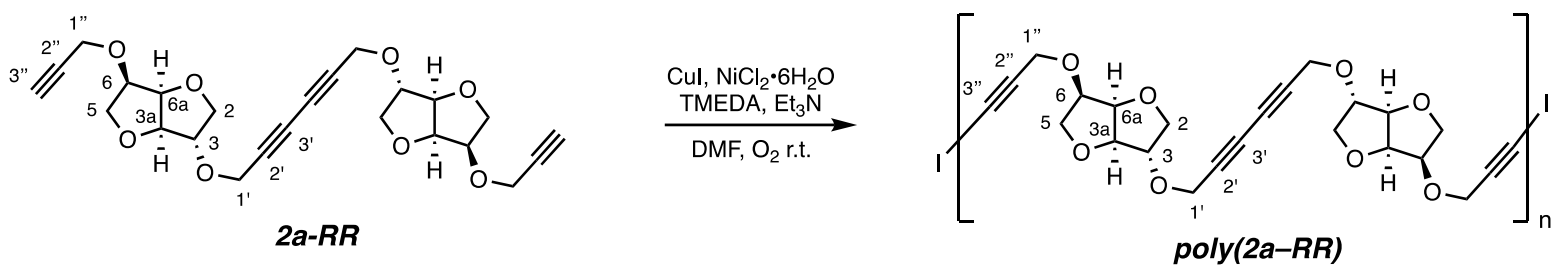

Poly(2a-RR) was recovered as a fluffy, off-white powder (155 mg from $200 \mathrm{mg}$ of $\mathbf{8}$; $78 \%$ yield).

${ }^{1}$ H NMR $\left(500 \mathrm{MHz}, \mathrm{DMSO}-d_{6}\right): \delta 4.60(\mathrm{dd}, J=4.7,4.7 \mathrm{~Hz}, 1 \mathrm{H}, H 3 \mathrm{a}), 4.48$ (ddd, $J=4.4,1.0,1.0 \mathrm{~Hz}$, $1 \mathrm{H}, H 6 \mathrm{a}), 4.43\left(\mathrm{~d} J=16.6 \mathrm{~Hz}, 1 \mathrm{H}, \mathrm{C} 1^{\prime} H_{\mathrm{a}} \mathrm{H}_{\mathrm{b}}\right), 4.40\left(\mathrm{~s}, 1 \mathrm{H}, \mathrm{C} 1^{\prime} H_{2}\right), 4.36$ (d, $\left.J=16.6 \mathrm{~Hz}, 1 \mathrm{H}, \mathrm{C} 1^{\prime} \mathrm{H}_{\mathrm{a}} H_{\mathrm{b}}\right)$, 4.14 (ddd, $J=6.7,4.7,4.7 \mathrm{~Hz}, 1 \mathrm{H}, H 3), 4.06$ (ddd, $J=3.8,1.9,1.2 \mathrm{~Hz}, 1 \mathrm{H}, H 6), 3.89$ (ddd, $J=10.4,1.2$, $\left.1.2 \mathrm{~Hz}, 1 \mathrm{H}, \mathrm{C} 5 \mathrm{H}_{\mathrm{a}} \mathrm{H}_{\mathrm{b}}\right), 3.82\left(\mathrm{dd}, J=8.9,6.4 \mathrm{~Hz}, 1 \mathrm{H}, \mathrm{C} 2 \mathrm{H}_{\mathrm{a}} \mathrm{H}_{\mathrm{b}}\right), 3.76\left(\mathrm{dd}, J=10.3,3.7 \mathrm{~Hz}, 1 \mathrm{H}, \mathrm{C} 5 \mathrm{H}_{\mathrm{a}} H_{\mathrm{b}}\right)$, and $3.46\left(\mathrm{dd}, J=8.9,6.5 \mathrm{~Hz}, 1 \mathrm{H}, \mathrm{C} 2 \mathrm{H}_{\mathrm{a}} H_{\mathrm{b}}\right)$.

${ }^{13}$ C NMR (125 MHz, DMSO- $\left.d_{6}\right): \delta 85.6,83.4,80.3,79.3,77.1(\mathrm{C} 2$ ' or C2'’), 77.0(C2' or C2'’), 72.8, 70.06(C1' or $\mathrm{C} 1$ ''), 70.02, 69.98(C1' or $\mathrm{C} 1$ ''), 57.8, and 57.0.

SEC-MALS $M_{\mathrm{n}}=15.7 \mathrm{kDa}, \emptyset=1.7$.

DSC $T_{\mathrm{m}}=-28^{\circ} \mathrm{C}, T_{\mathrm{g}}=23^{\circ} \mathrm{C}$.

TGA $T_{\mathrm{d} 5 \%}=178^{\circ} \mathrm{C}$.

$¥$ the descriptor "RR" in $\mathbf{2 a - R R}$ [and poly(2a-RR)] designates that this is a regioregular monomer (and polymer 


\section{Typical procedure for the hydrogenation of polymeric diynes poly $(2 a-c)$.}

To a solution of poly(2a) $(100 \mathrm{mg})$ in $2 \mathrm{~mL}$ of dimethylformamide in a small vial equipped with a stir bar was added $\mathrm{Pd} / \mathrm{C}(20 \mathrm{wt} \%$, ca. $20 \mathrm{mg})$ and potassium carbonate $(50 \mathrm{wt} \%, 50 \mathrm{mg})$. The vial was placed into a $100 \mathrm{~mL}$ Fischer-Porter vessel, which was sealed and twice pressurized with hydrogen gas and vented. The vessel was again pressurized to $80 \mathrm{psi}$, and the black suspension was allowed to stir for 1 week. The pressure was vented, and the suspension was filtered through a pad of Celite ${ }^{\circledR}$, and the pad was washed with a small amount of additional DMF. The filtrate was concentrated in vacuo to afford poly(2a)-H as a sticky, lightly colored solid ( $87 \mathrm{mg}, 89 \%$ isolated yield).

$\operatorname{Poly}(2 a)-[H]$

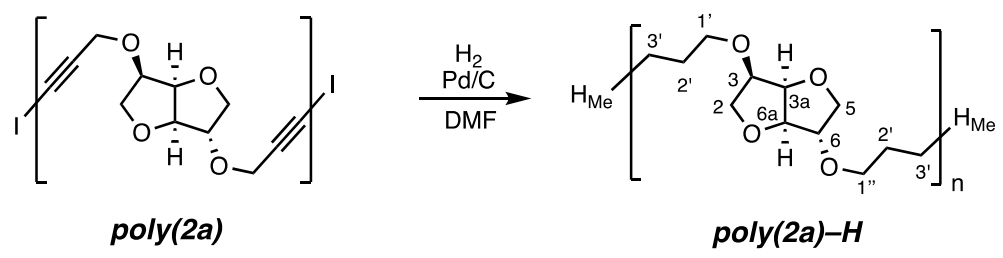

${ }^{1}$ H NMR (500 MHz, DMSO- $\left.d_{6}\right): \delta 4.52(\mathrm{dd}, J=4.6,4.6 \mathrm{~Hz}, 1 \mathrm{H}, H 3 \mathrm{a}), 4.39$ (brd, $\left.J=4.4 \mathrm{~Hz}, 1 \mathrm{H}, H 6 \mathrm{a}\right)$, 3.93 (ddd, $J=7.0,7.0,4.5, \mathrm{~Hz}, 1 \mathrm{H}, H 3), 3.85$ (brd, $J=3.6 \mathrm{~Hz}, 1 \mathrm{H}, \mathrm{H} 6), 3.79$ (brd, $J=8.9 \mathrm{~Hz}, 1 \mathrm{H}, \mathrm{C} 5 H_{\mathrm{a}} \mathrm{H}_{\mathrm{b}}$ ), $3.76\left(\mathrm{dd}, J=9.9,3.8 \mathrm{~Hz}, 1 \mathrm{H}, \mathrm{C} 5 \mathrm{H}_{\mathrm{a}} H_{\mathrm{b}}\right), 3.55$ (dddd, $\left.J=9.1,6.7,6.7,0.6 \mathrm{~Hz}, 1 \mathrm{H}, \mathrm{OC}^{\prime} H_{\mathrm{a}} \mathrm{H}_{\mathrm{b}}\right), 3.43-3.38$ $\left(\mathrm{m}, 3 \mathrm{H}, \mathrm{OC}{ }^{\prime}{ }^{\prime} H_{2}\right.$ and $\left.\mathrm{C} 5 \mathrm{H}_{\mathrm{a}} H_{\mathrm{b}}\right), 3.37$ (ddd, $\left.J=8.8,6.6,6.6 \mathrm{~Hz}, 1 \mathrm{H}, \mathrm{OC} 1{ }^{\prime} \mathrm{H}_{\mathrm{a}} H_{\mathrm{b}}\right), 1.51-1.46\left(\mathrm{~m}, 4 \mathrm{H}, \mathrm{C} 2^{\prime} \mathrm{H}_{2}\right.$ and $\left.\mathrm{C} 2{ }^{\prime}{ }^{\prime} \mathrm{H}_{2}\right)$, and $1.32-1.27\left(\mathrm{~m}, 4 \mathrm{H}, \mathrm{C} 3{ }^{\prime} \mathrm{H}_{2}\right.$ and $\left.\mathrm{C} 3{ }^{\prime} \mathrm{H}_{2}\right)$. Resonances for the two methyl end groups were observed at $0.860\left(\mathrm{t}, J=7.4 \mathrm{~Hz}, 0.12 \mathrm{H}, \mathrm{C}^{\prime}{ }^{\prime} H_{3}\right.$ or C3' ' $\left.H_{3}\right)$ and $0.856\left(\mathrm{t}, J=7.4 \mathrm{~Hz}, 0.12 \mathrm{H}, \mathrm{C} 3{ }^{\prime} H_{3}\right.$ or C3' ' $\left.H_{3}\right)$.

${ }^{13}$ C NMR (125 MHz, DMSO- $\left.d_{6}\right)$ : $\delta 86.0,84.2,80.2,80.1,73.0,69.9(\mathrm{C} 1$ ' or C1' '), 69.8(C1' or C1' '), 69.0, 29.8, 29.7, and 25.9.

SEC-MALS $M_{\mathrm{n}}=9.7 \mathrm{kDa}, \emptyset=1.6$.

DSC $T_{\mathrm{g}}=-10^{\circ} \mathrm{C}$.

TGA $T_{\mathrm{d} 5 \%}=289^{\circ} \mathrm{C}$. 
$\operatorname{Poly}(2 b)-[H]$

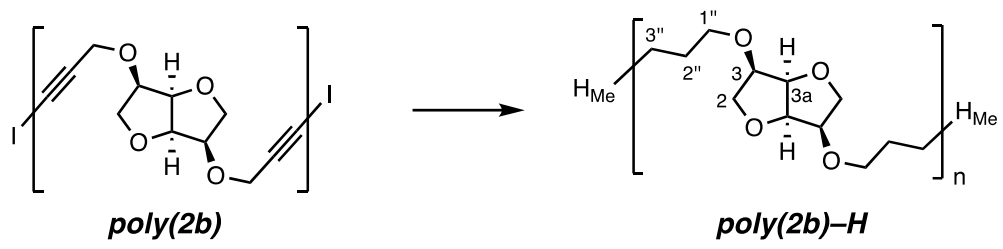

Poly(2b)-H was recovered as a sticky, lightly colored solid ( $88 \%$ isolated yield).

${ }^{1}$ H NMR (500 MHz, DMSO- $d_{6}$ ): $\delta 4.48$ (nfom, 2H, H3a), 3.93 (dddd, $J=8.3,7.0,3.4,1.6 \mathrm{~Hz}, 2 \mathrm{H}, H 3$ ), $3.87\left(\mathrm{dd}, J=8.2,6.9 \mathrm{~Hz}, 2 \mathrm{H}, \mathrm{C} 2 \mathrm{H}_{\mathrm{a}} \mathrm{H}_{\mathrm{b}}\right), 3.52\left(\mathrm{dt}, J=15.7,6.7 \mathrm{~Hz}, 2 \mathrm{H}, \mathrm{Cl}{ }^{\prime} H_{\mathrm{a}} \mathrm{H}_{\mathrm{b}}\right), 3.41(\mathrm{dd}, J=8.2,8.2 \mathrm{~Hz}$, $\left.2 \mathrm{H}, \mathrm{C} 2 \mathrm{H}_{\mathrm{a}} H_{\mathrm{b}}\right), 3.37\left(\mathrm{dt}, J=15.7,6.5, \mathrm{~Hz}, \mathrm{C} 1{ }^{\prime} \mathrm{H}_{\mathrm{a}} H_{\mathrm{b}} 2 \mathrm{H}\right), 1.51-1.46\left(\mathrm{~m}, 4 \mathrm{H}, \mathrm{C} 2{ }^{\prime} \mathrm{H}_{2}\right)$, and $1.32-1.27(\mathrm{~m}, 4 \mathrm{H}$, $\left.\mathrm{C}^{\prime}{ }^{\prime} \mathrm{H}_{2}\right)$. Resonances for the two methyl endgroups were observed at $0.860\left(\mathrm{t}, J=7.4 \mathrm{~Hz}, 0.20 \mathrm{H}, \mathrm{C}^{\prime}{ }^{\prime} \mathrm{H}_{3}\right.$ or $\left.\mathrm{C} 3{ }^{\prime} \mathrm{H}_{3}\right)$

${ }^{13}$ C NMR (125 MHz, DMSO- $\left.d_{6}\right): \delta 80.3,80.2,70.8,69.9(\mathrm{C} 1 ’ ’), 29.8$, and 25.9.

SEC-MALS $M_{\mathrm{n}}=4.3 Ð=1.3$.

DSC $T_{\mathrm{g}}=-2{ }^{\circ} \mathrm{C}$.

TGA $T_{\mathrm{d} 5 \%}=208^{\circ} \mathrm{C}$.

$\operatorname{Poly}(2 \mathrm{c})-[\mathrm{H}]$

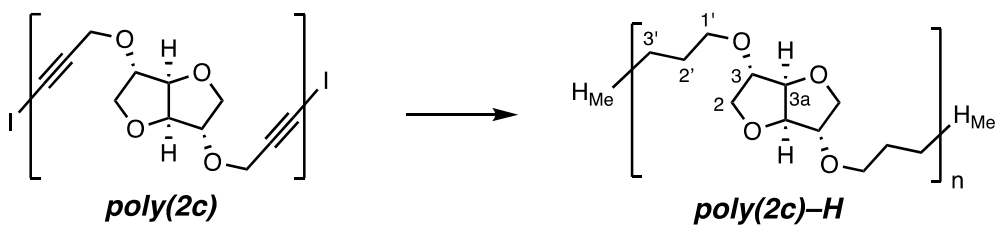

Poly(2c)-H was recovered as a sticky, lightly colored solid (72\% isolated yield).

${ }^{1}$ H NMR (500 MHz, DMSO- $\left.d_{6}\right): \delta 4.41$ (br s, 2H, H3a), 3.86-3.83 (m, 2H, H3), 3.71-3.67 (m, 4H, C2 $\left.H_{2}\right)$, $3.47-3.42\left(\mathrm{~m}, 4 \mathrm{H}, \mathrm{C} 1^{\prime} H_{2}\right), 3.39\left(\mathrm{dt}, J=15.7,6.5, \mathrm{~Hz}, \mathrm{C} 1{ }^{\prime} \mathrm{H}_{\mathrm{a}} H_{\mathrm{b}} 2 \mathrm{H}\right), 1.53-1.45\left(\mathrm{~m}, 4 \mathrm{H}, \mathrm{C} 2{ }^{\prime} \mathrm{H}_{2}\right)$, and $1.34-$ $1.28\left(\mathrm{~m}, 4 \mathrm{H}, \mathrm{C}^{\prime} \mathrm{H}_{2}\right)$. Resonances for the methyl end groups were observed at $0.86(\mathrm{t}, J=7.4 \mathrm{~Hz}, 0.14 \mathrm{H}$, C3' $\left.\mathrm{H}_{3}\right)$.

${ }^{13}$ C NMR (125 MHz, DMSO- $\left.d_{6}\right): \delta 85.3,83.4,71.8,69.1,29.7$, and 25.9.

SEC-MALS $M_{\mathrm{n}}=5.5 \mathrm{kDa}, \emptyset=1.8$.

DSC $T_{\mathrm{g}}=-9{ }^{\circ} \mathrm{C} ; T_{\mathrm{m}}{ }^{1}=-29{ }^{\circ} \mathrm{C} ; T_{\mathrm{m}}{ }^{2}=83{ }^{\circ} \mathrm{C}$.

TGA $T_{\mathrm{d} 5 \%}=248^{\circ} \mathrm{C}$. 
IV. Polymer DSC, TGA, SEC traces:

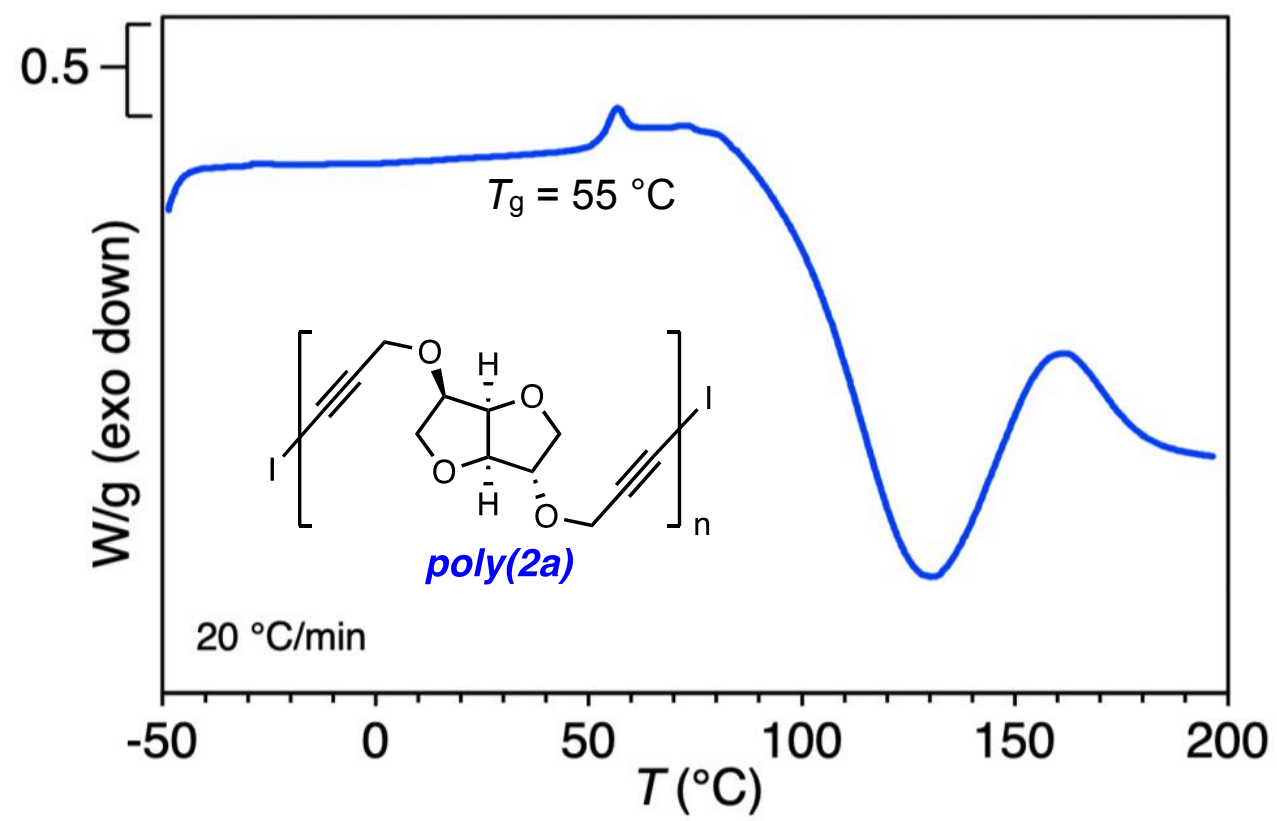

Figure S1. DSC thermogram for poly(2a)

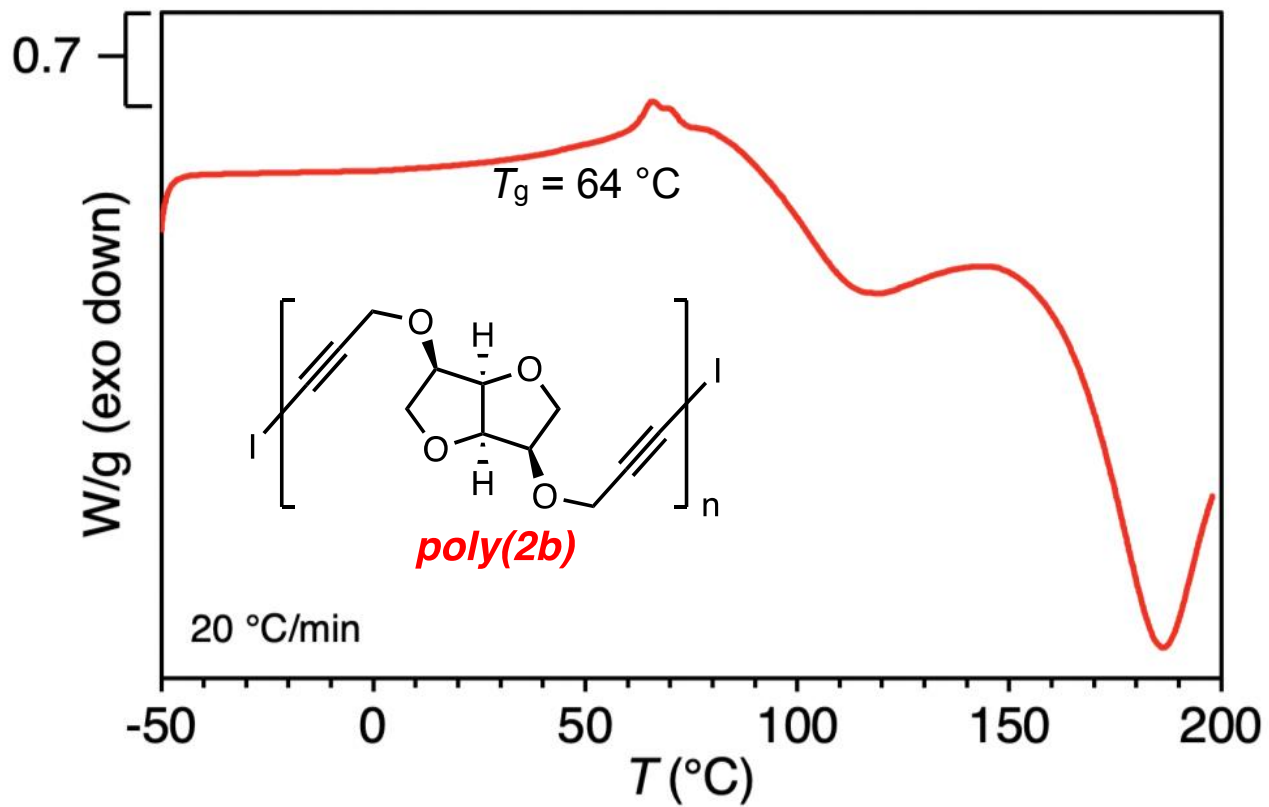

Figure S2. DSC thermogram for poly(2b) 


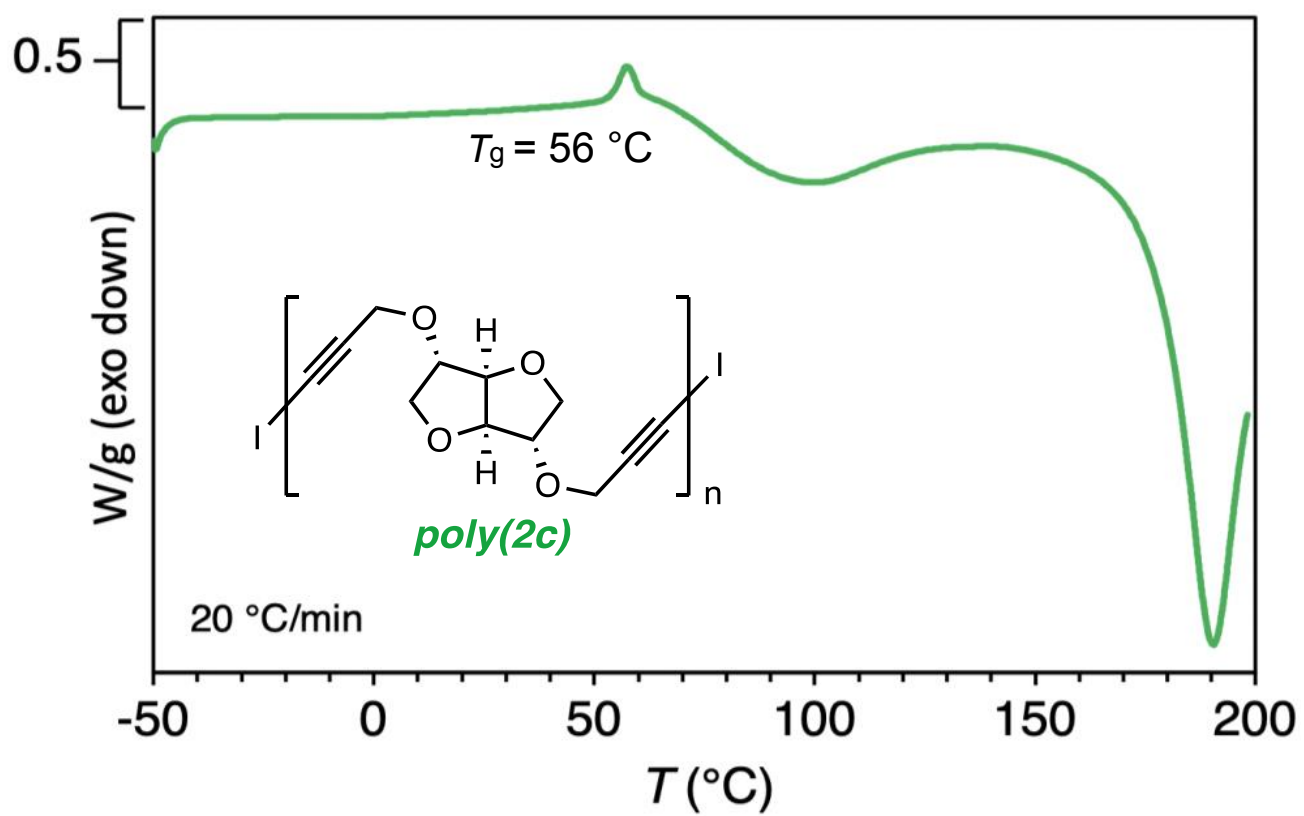

Figure S3. DSC thermogram for poly(2c)

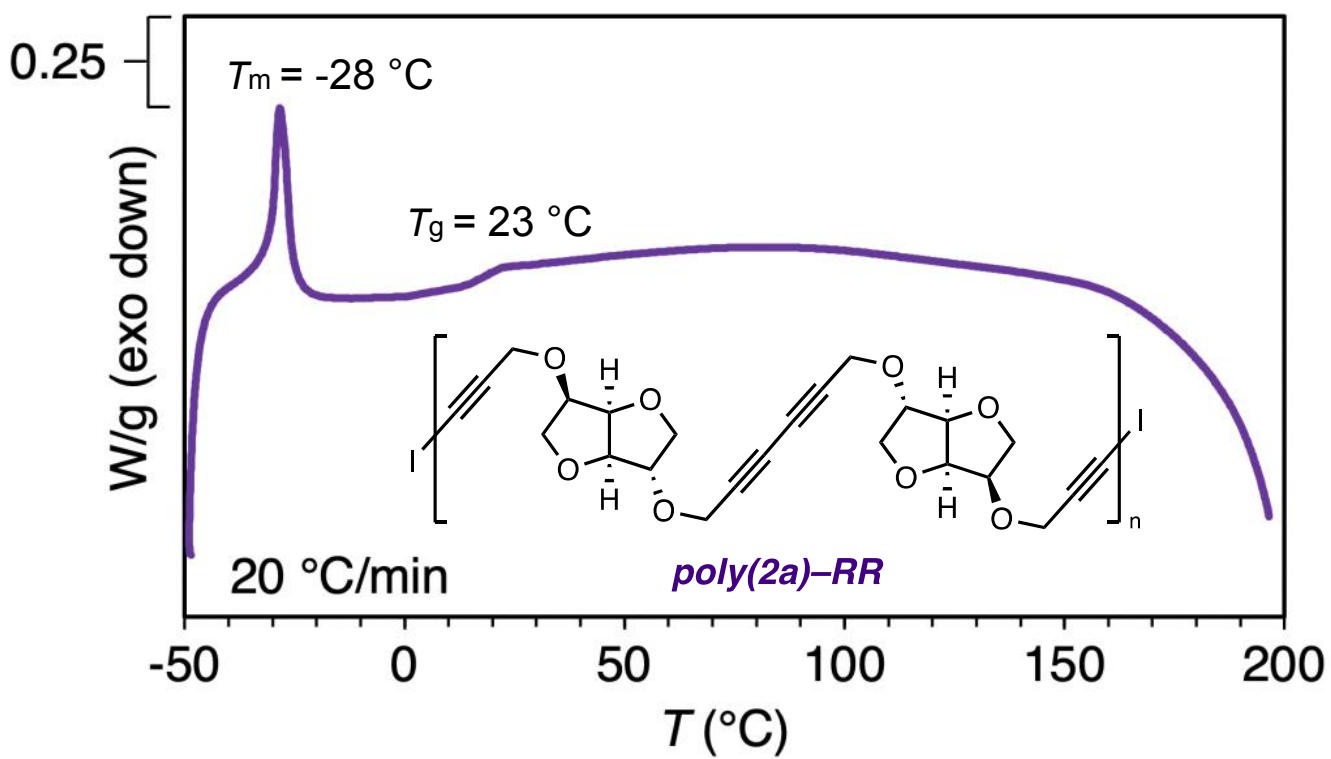

Figure S4. DSC thermogram for poly $(2 \mathbf{a}-\mathbf{R R})$ 


\section{Brief discussion of the thermal properties (DSC) of Poly(2a-RR}

We also explored the effects of regioregularity on the isosorbide-based diyne polymer. That is, what effect would an all-head-to-head orientation of the diynes in the polyne backbone have on the thermal properties? To that end, we prepared the bis-terminal diyne monomer $\mathbf{2 a - R R}$ already containing a pre-formed exo/exo 1,3-diyne linking the two isosorbide units. This can only polymerize through its terminal endo-propargyl ethers, resulting in alternating exo/exo- and endo/endo-2,4-hexadiynyl subunits between its isosorbide linkages. The ${ }^{1} \mathrm{H}$ and ${ }^{13} \mathrm{C} \mathrm{NMR}$ spectral properties of poly(2a) and poly(2a-RR) are nearly identical, except for subtle, but diagnostic, differences in the diyne carbon resonances, but the thermal behaviors of these two polymers are remarkably different. While poly(2a) displays no $T_{\mathrm{m}}$ and a $T_{\mathrm{g}}$ of $55^{\circ} \mathrm{C}$ (Figure $\mathrm{S} 1$ ), poly(2a-RR) displays a strong melting transition at $-28{ }^{\circ} \mathrm{C}$ and a much lower $T_{\mathrm{g}}$ of $23{ }^{\circ} \mathrm{C}$ (Figure S4). Although we do not have a strong rationale to offer in way of explanation, these data highlight the profound effect that subtle changes in polymer microstructure can have on the thermal properties of the material, at least in the context of isosorbide-based polymers. 


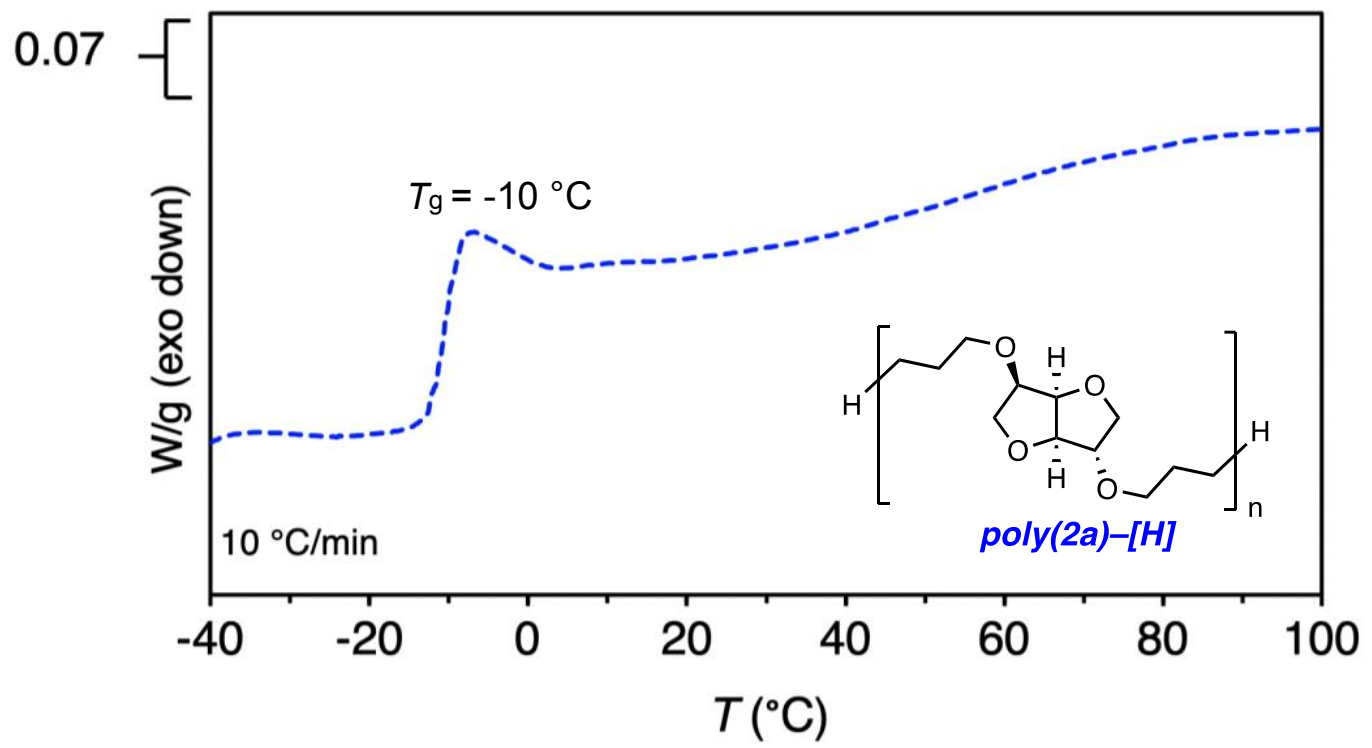

Figure S5. DSC thermogram for poly(2a)-[H]

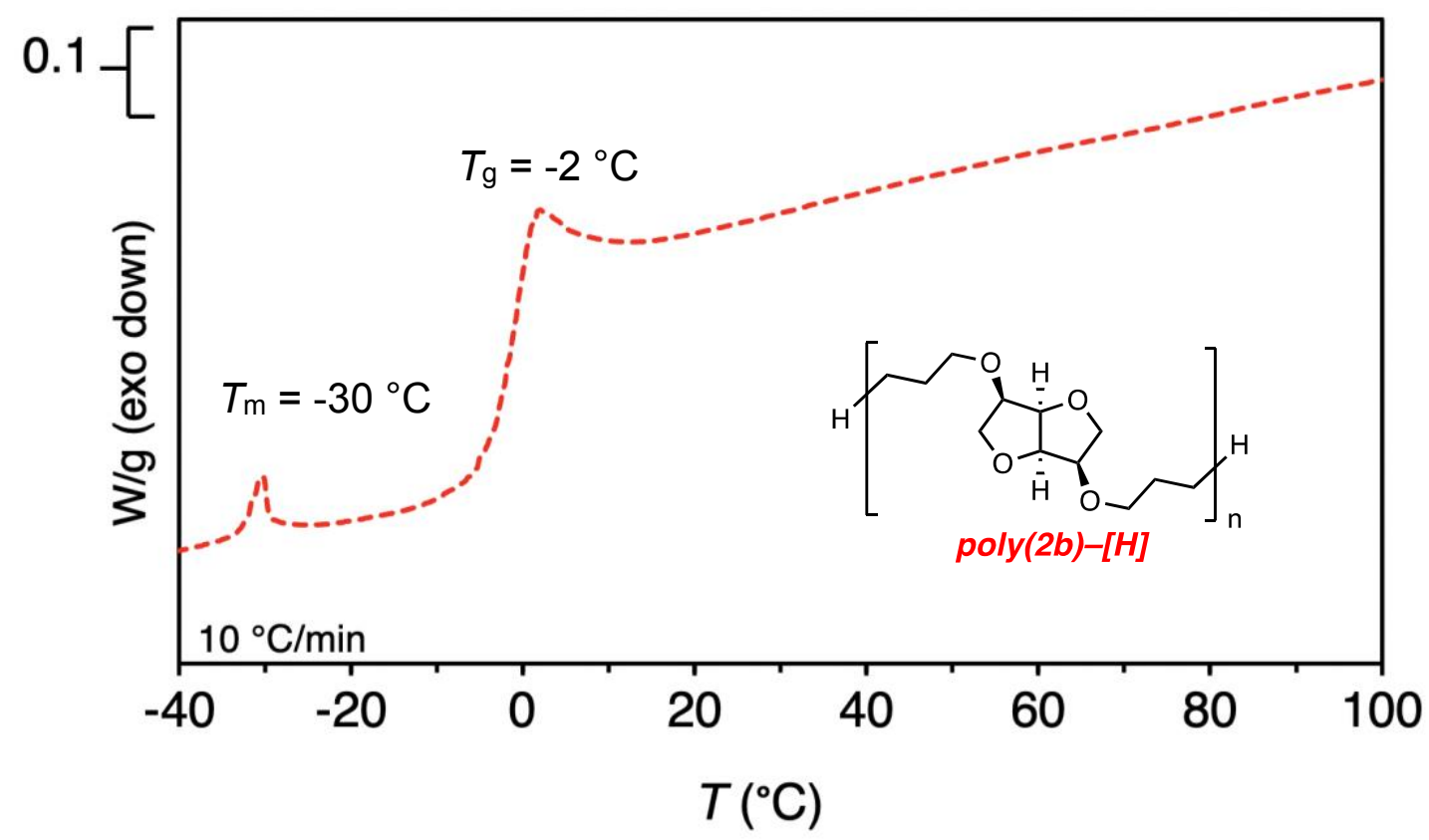

Figure S6. DSC thermogram for poly(2b)-[H] 


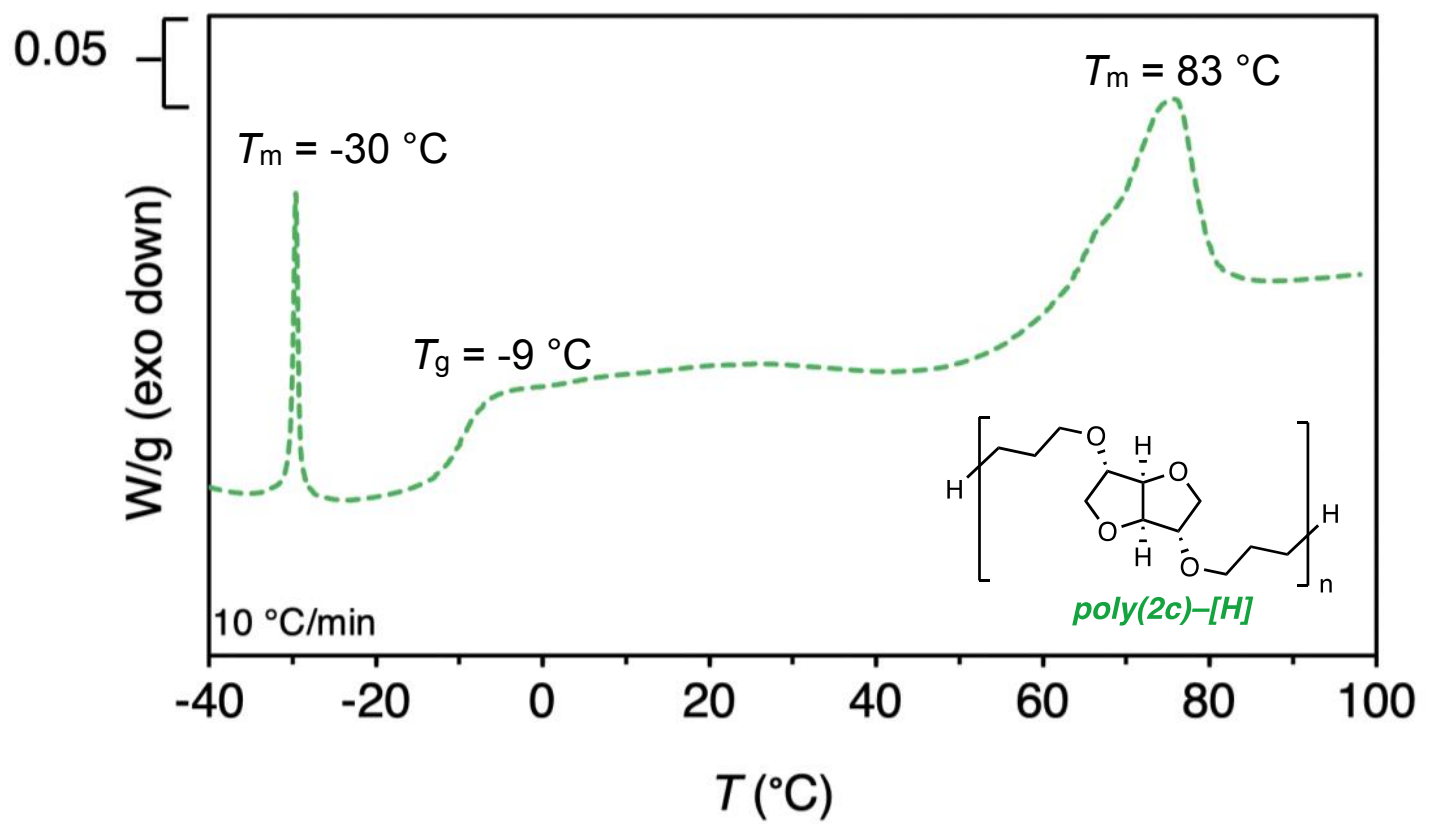

Figure S7. DSC thermogram for poly(2c)-[H]

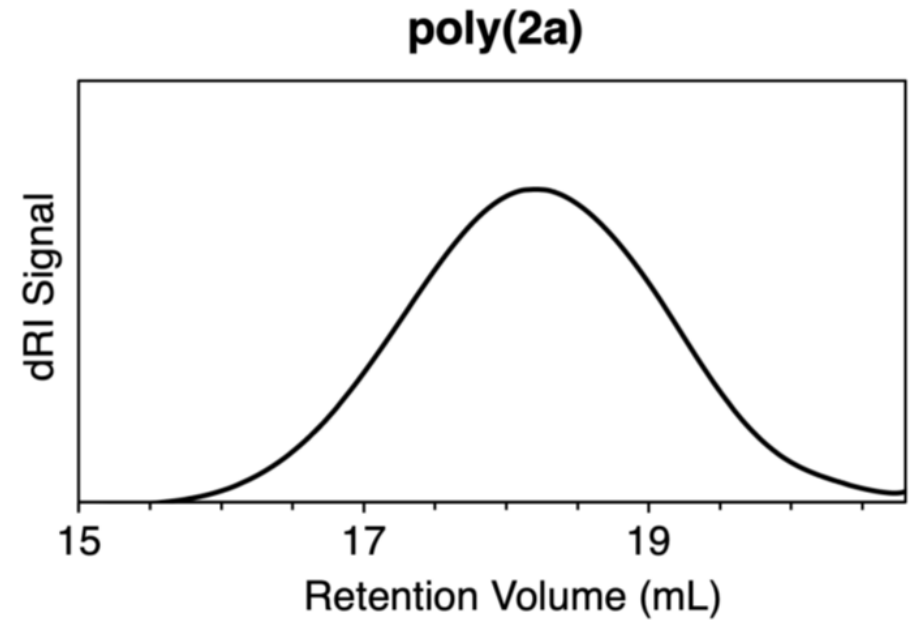

Figure S8. SEC chromatogram (DMF mobile phase) for poly(2a) 


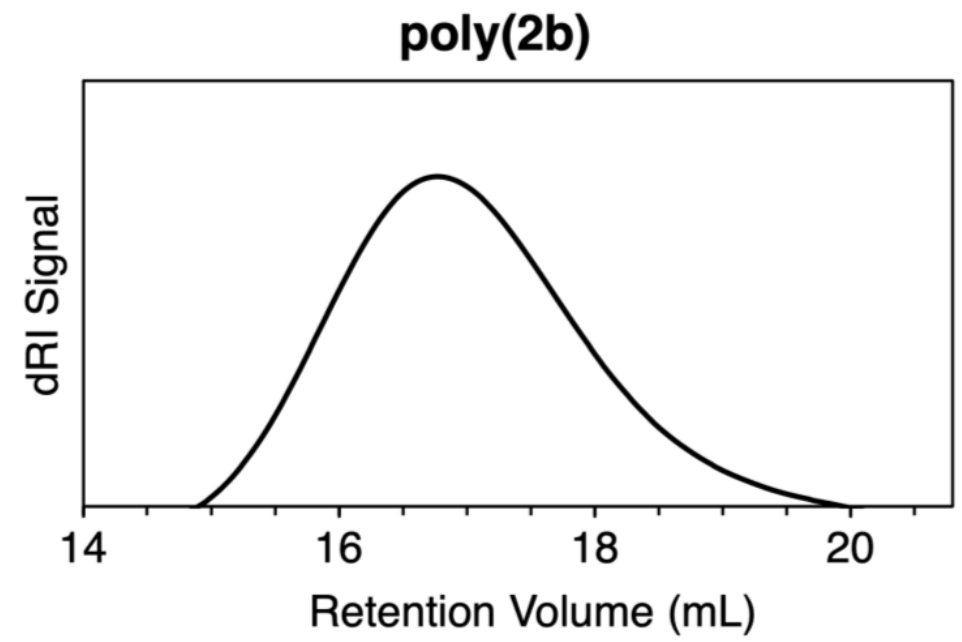

Figure S9. SEC chromatogram (DMF mobile phase) for poly(2b)

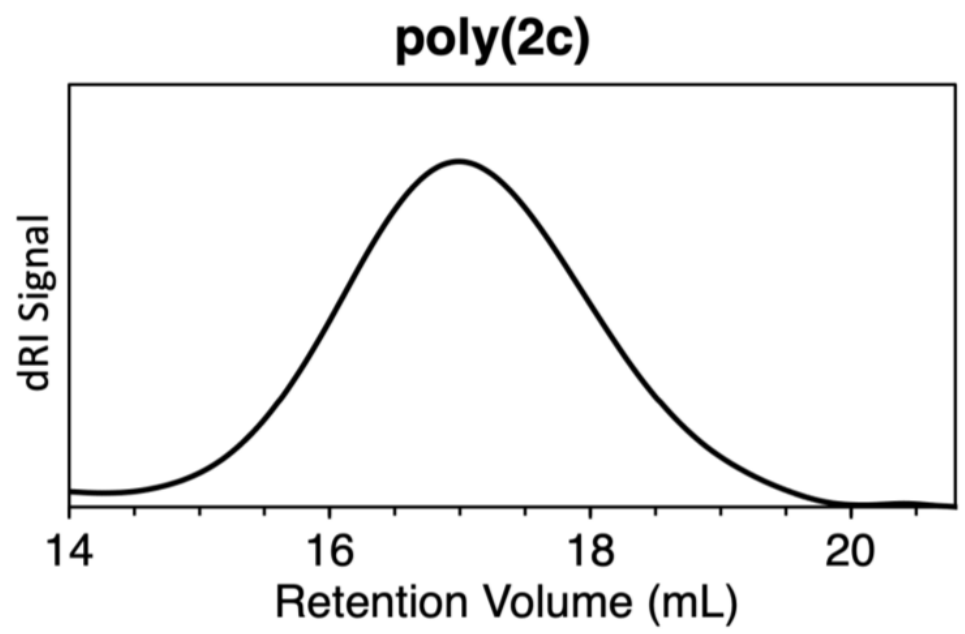

Figure S10. SEC chromatogram (DMF mobile phase) for poly(2c) 


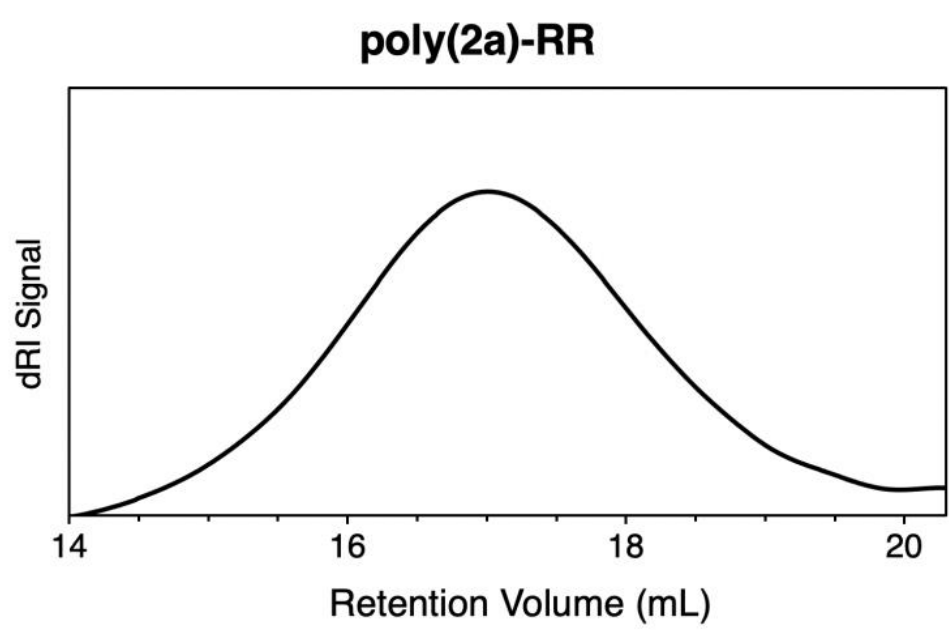

Figure S11. SEC chromatogram (DMF mobile phase) for poly(2a-RR)

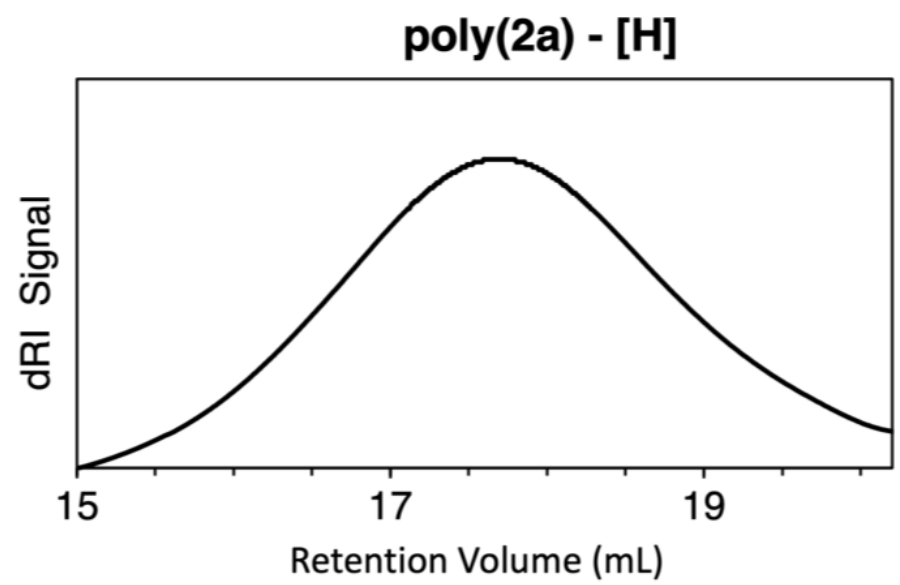

Figure S12. SEC chromatogram (DMF mobile phase) for poly(2a)-[H] 


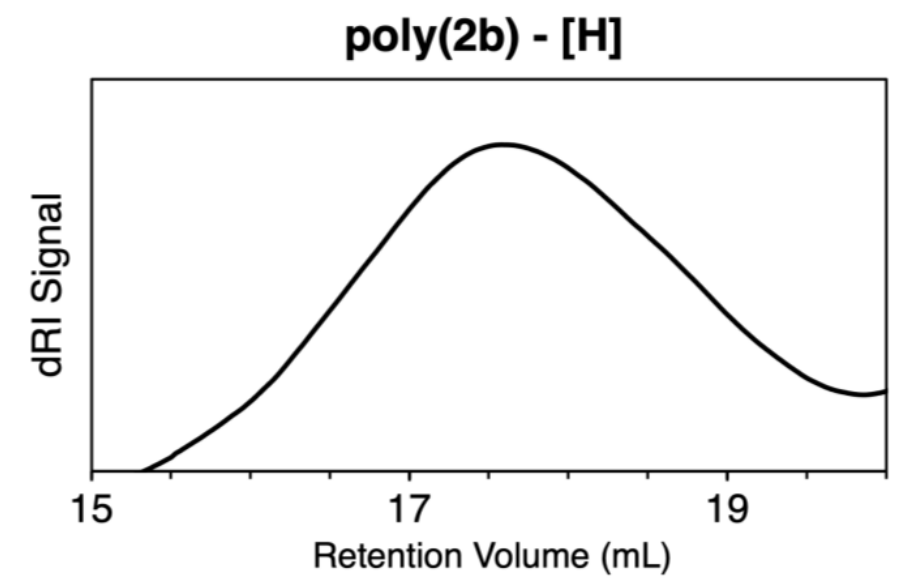

Figure S13. SEC chromatogram (DMF mobile phase) for poly(2b)-[H]

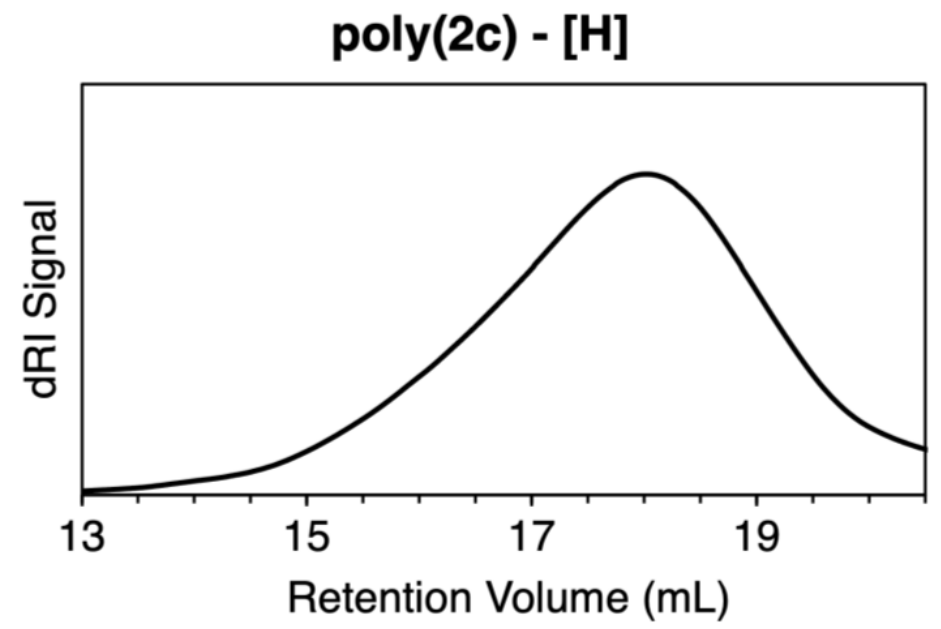

Figure S14. SEC chromatogram (DMF mobile phase) for poly(2c)-[H] 


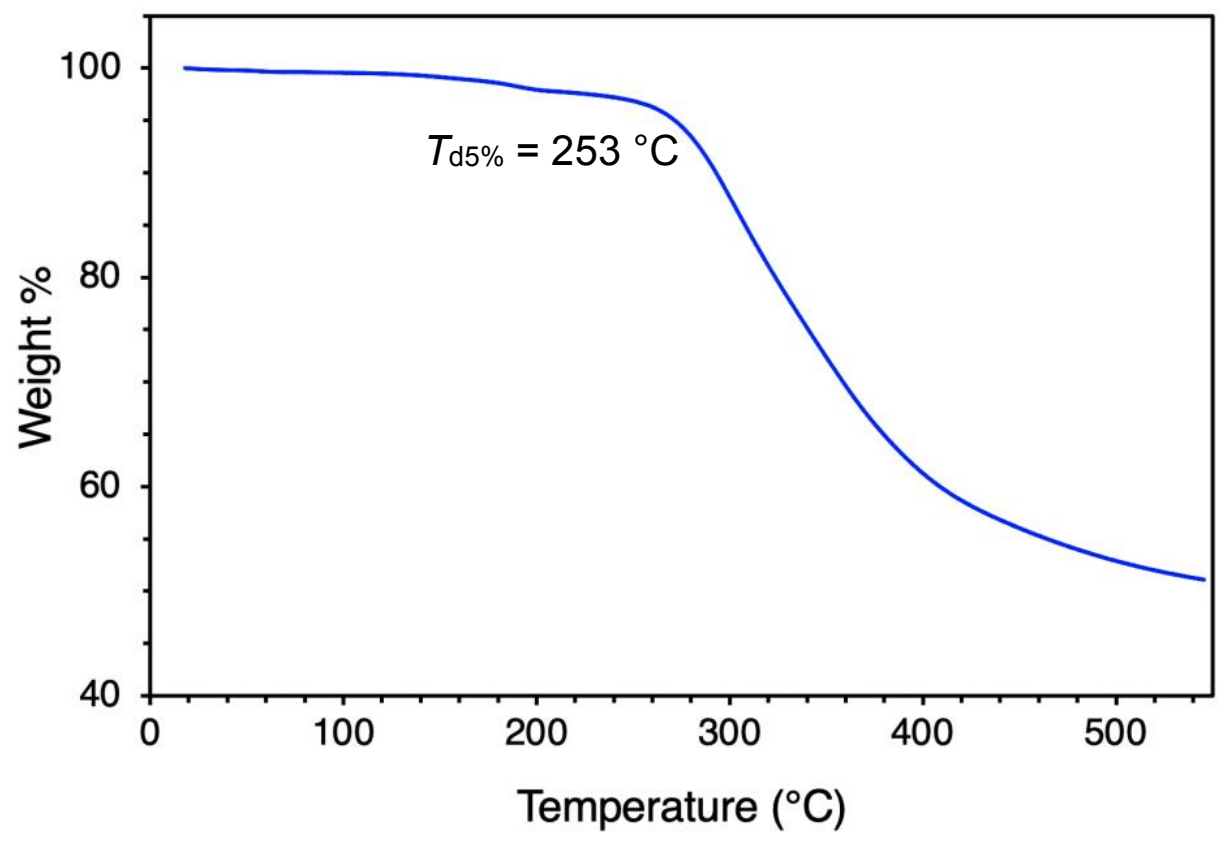

Figure S15. TGA thermogram for poly(2a)

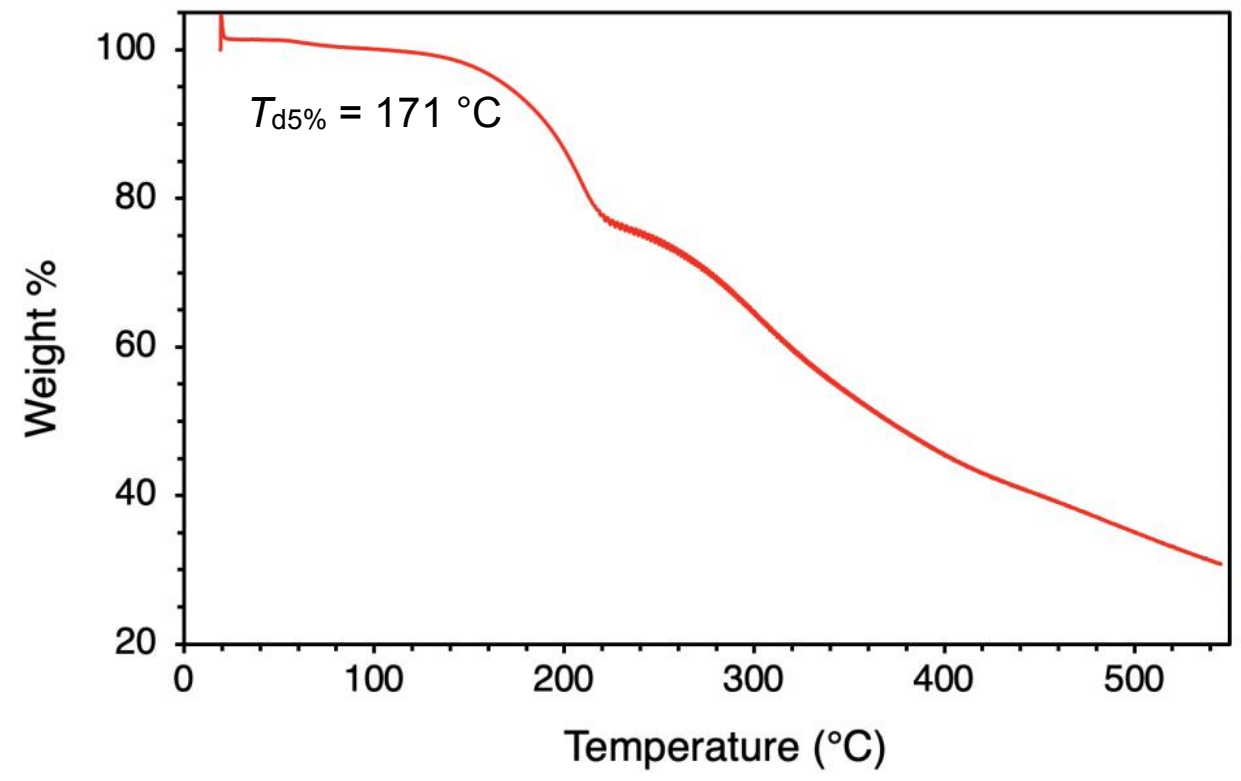

Figure S16. TGA thermogram for poly(2b) 


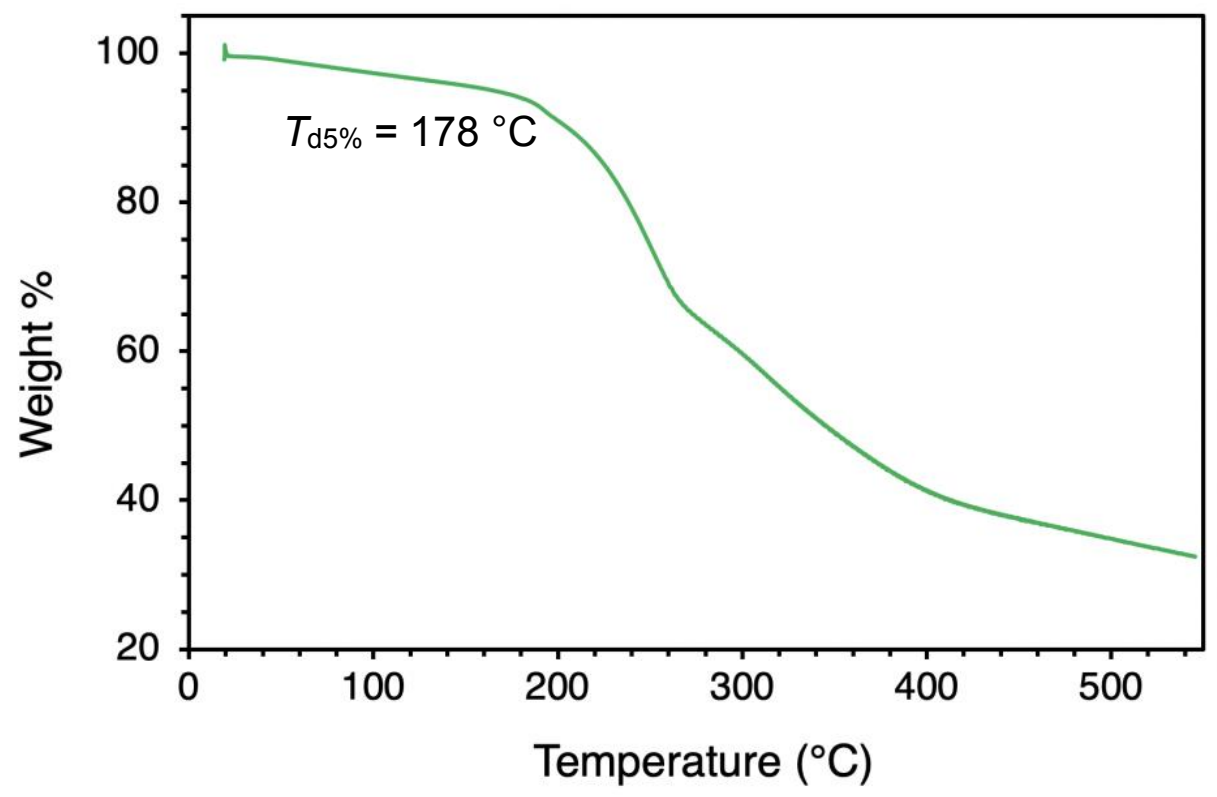

Figure S17. TGA thermogram for poly(2c)

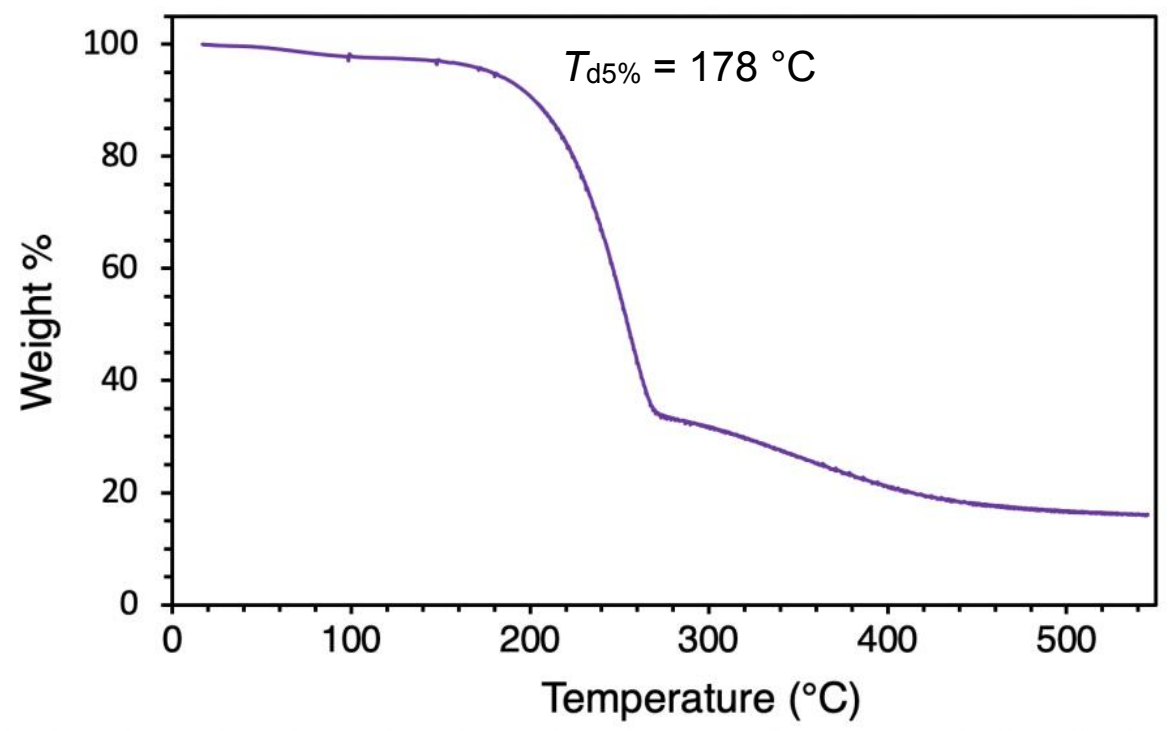

Figure S18. TGA thermogram for $\operatorname{poly}(2 a-R R)$ 


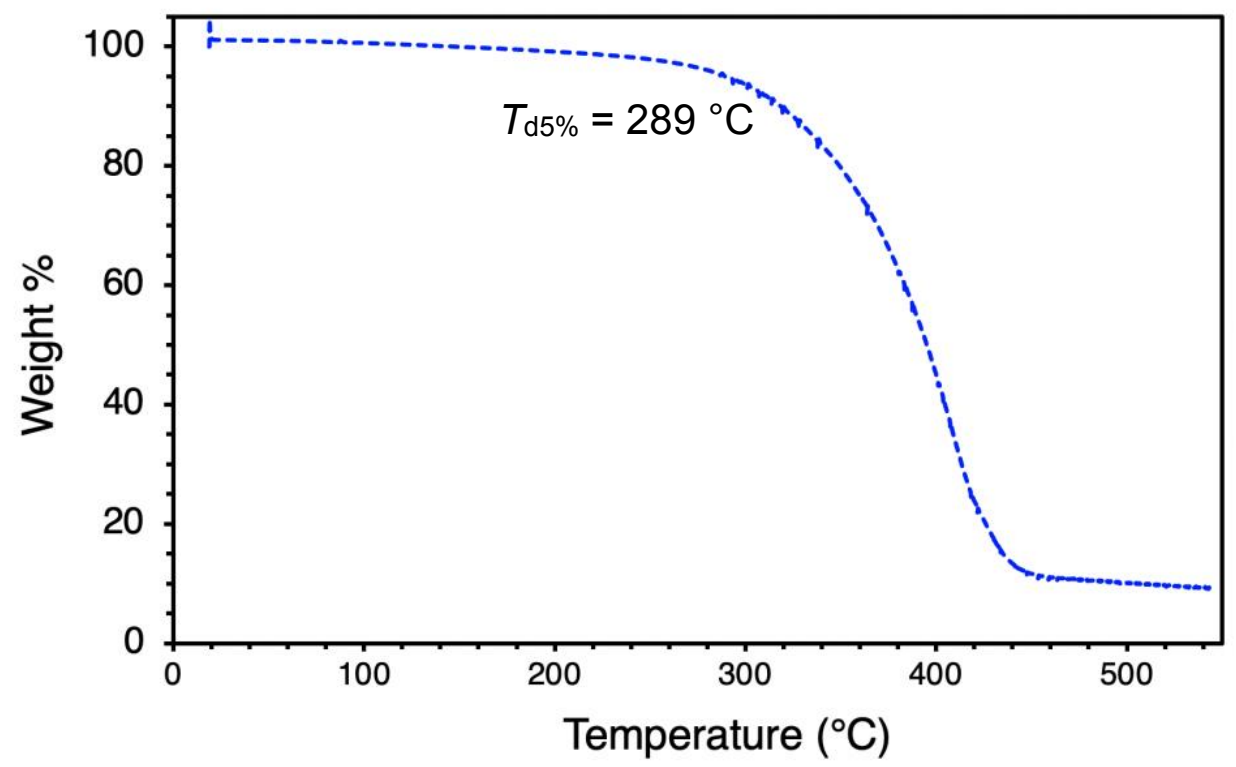

Figure S19. TGA thermogram for $\operatorname{poly}(2 \mathbf{a})-[\mathbf{H}]$

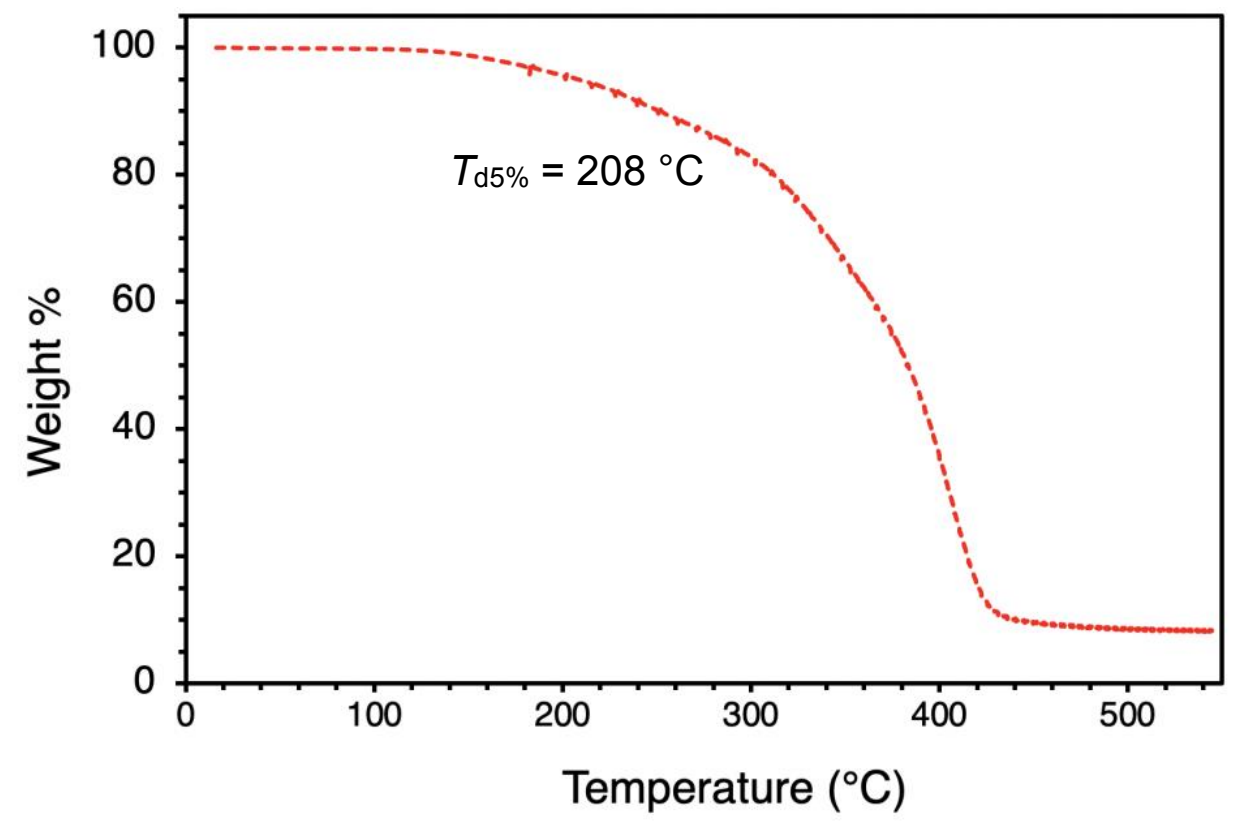

Figure S20. TGA thermogram for poly(2b)-[H] 


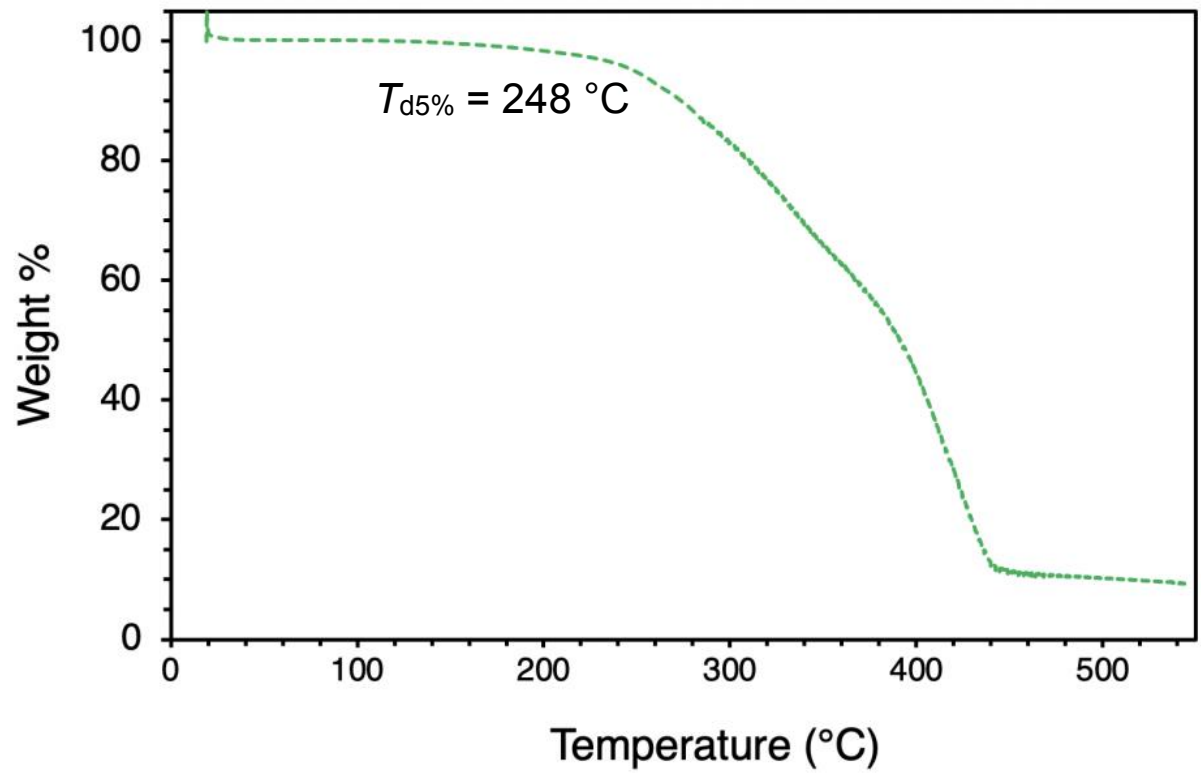

Figure S21. TGA thermogram for poly(2c)-[H] 


\section{Model study of aerobic oxidation of 4}

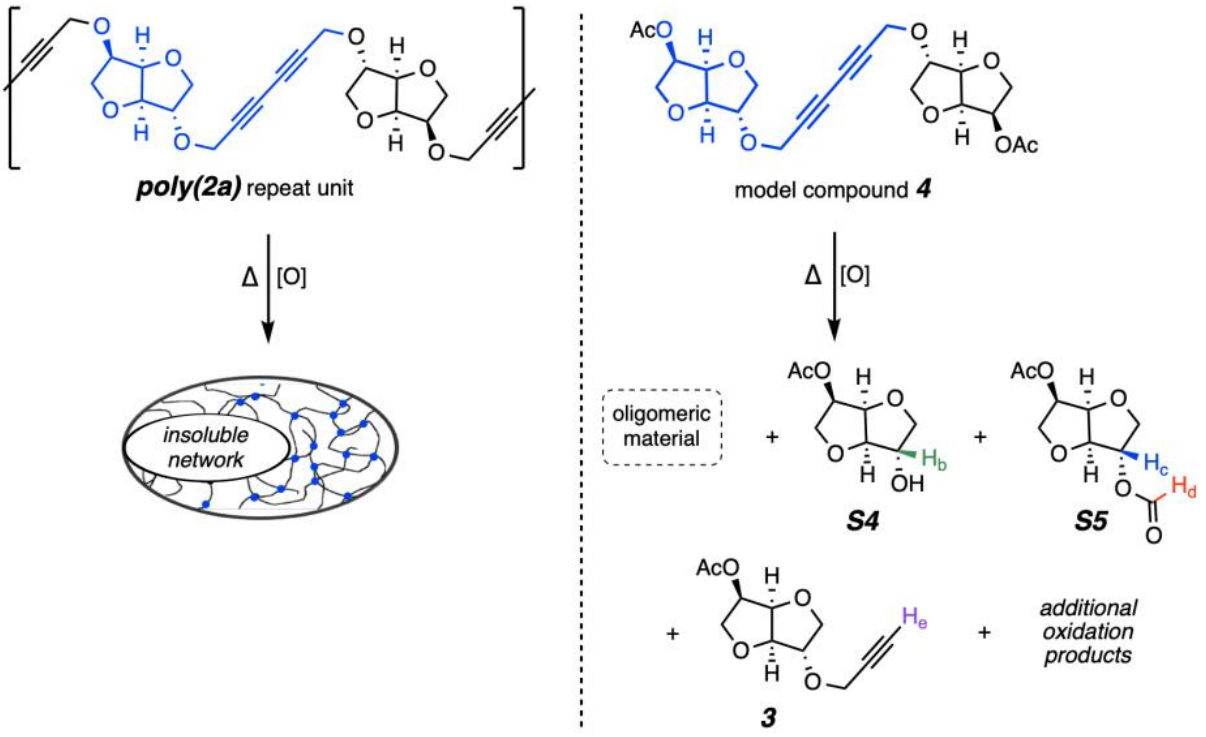

Figure S22. Model study for oxidation of poly(2a) using model compound 4.

The DSC thermograms of poly(2a-c) displayed large, irreversible exothermic events following the $T_{\mathrm{g}}$, beginning between $70-90{ }^{\circ} \mathrm{C}$ (see $\mathbf{S 2 3}$ ). The materials recovered from the DSC pans were insoluble in any organic solvent, and therefore challenging to characterize. In order to investigate the nature of this phenomenon, we devised a study using acetate-protected diyne 4, meant to simulate one repeat unit of the polymer, highlighted in blue (Figure S1). The model substrate was heated in bulk under a) a nitrogen atmosphere and b) an oxygen atmosphere for 24 hours. The crude products were dissolved in $\mathrm{CDCl}_{3}$ analyzed by GC-MS and ${ }^{1} \mathrm{H}$ NMR spectroscopy and then subjected to column chromatography. The suspected degradation products were then alternatively synthesized and fully characterized. 


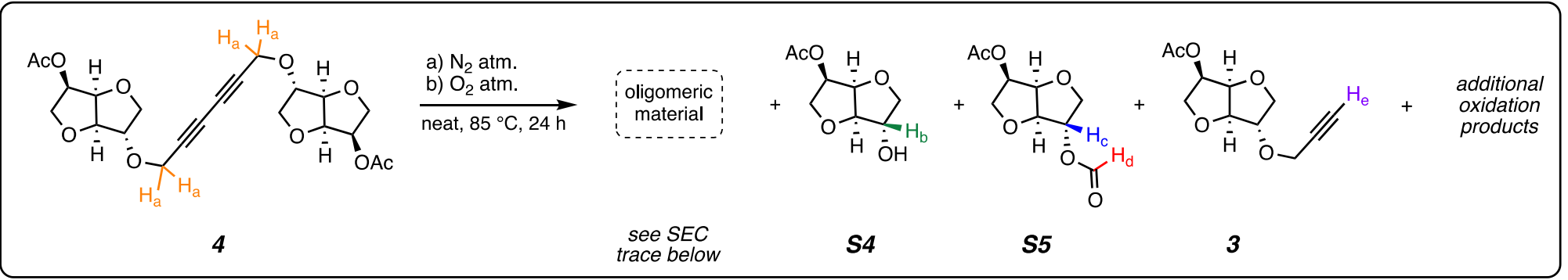

${ }^{1} \mathrm{H} \mathrm{NMR}, 500 \mathrm{MHz}, \mathrm{CDCl}_{3}$

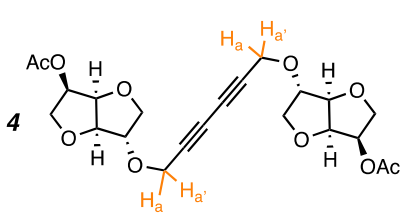

a) crude mixture from heating 4 under $\mathrm{N}_{2}$ atmosphere

b) crude mixture from heating 4 under $\mathrm{O}_{2}$ atmosphere
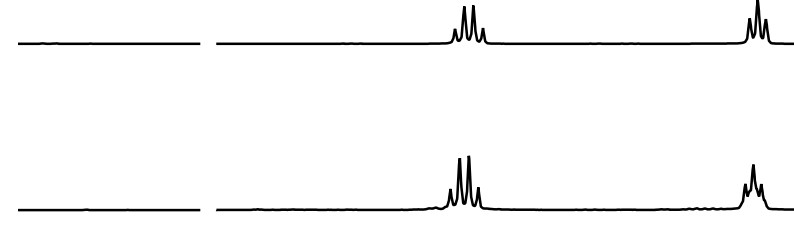

น

clumln

Mn

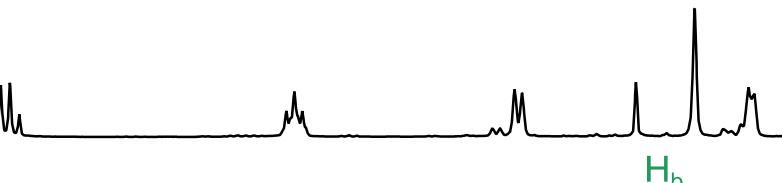

Nullun Mu $\mathrm{H}_{\mathrm{e}}$

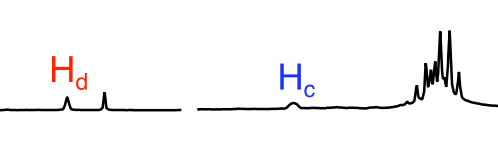

$M$

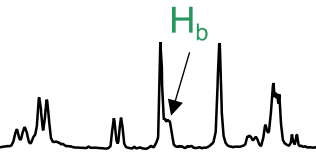<smiles>C=C(C)CCC(C)C</smiles>
$\mathrm{H}_{\mathrm{e}}$

isolated by chromatography and alt. synthesized

isolated by chromatography and alt. synthesized
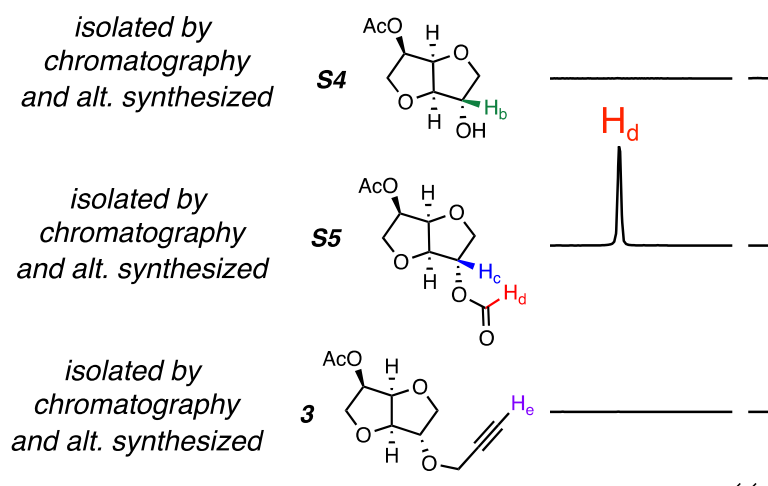

the
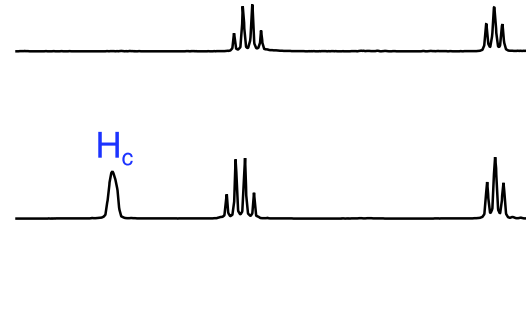

M

$\Lambda^{H_{b}}$<smiles>CC1CCCCC1</smiles>

Mn
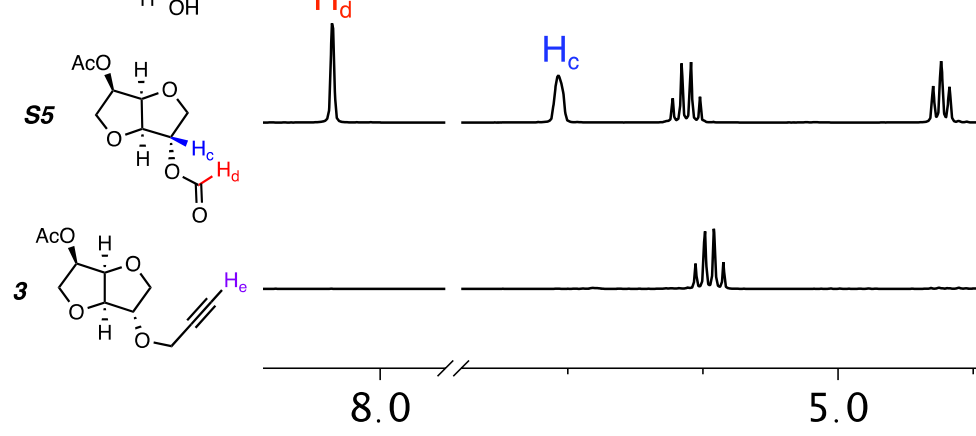

8.0
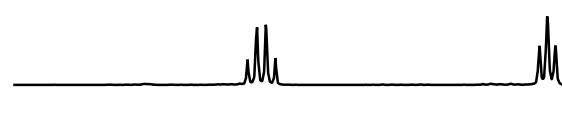

5.0

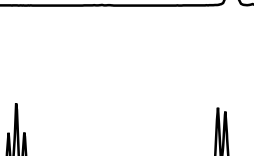

4.5

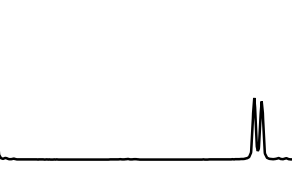

4.0

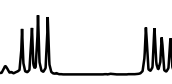

clllldu_ande

Figure S23. ${ }^{1}$ H NMR spectra of products S4, S5, and 3 resulting from thermal and/or oxidative decomposition of 4. 


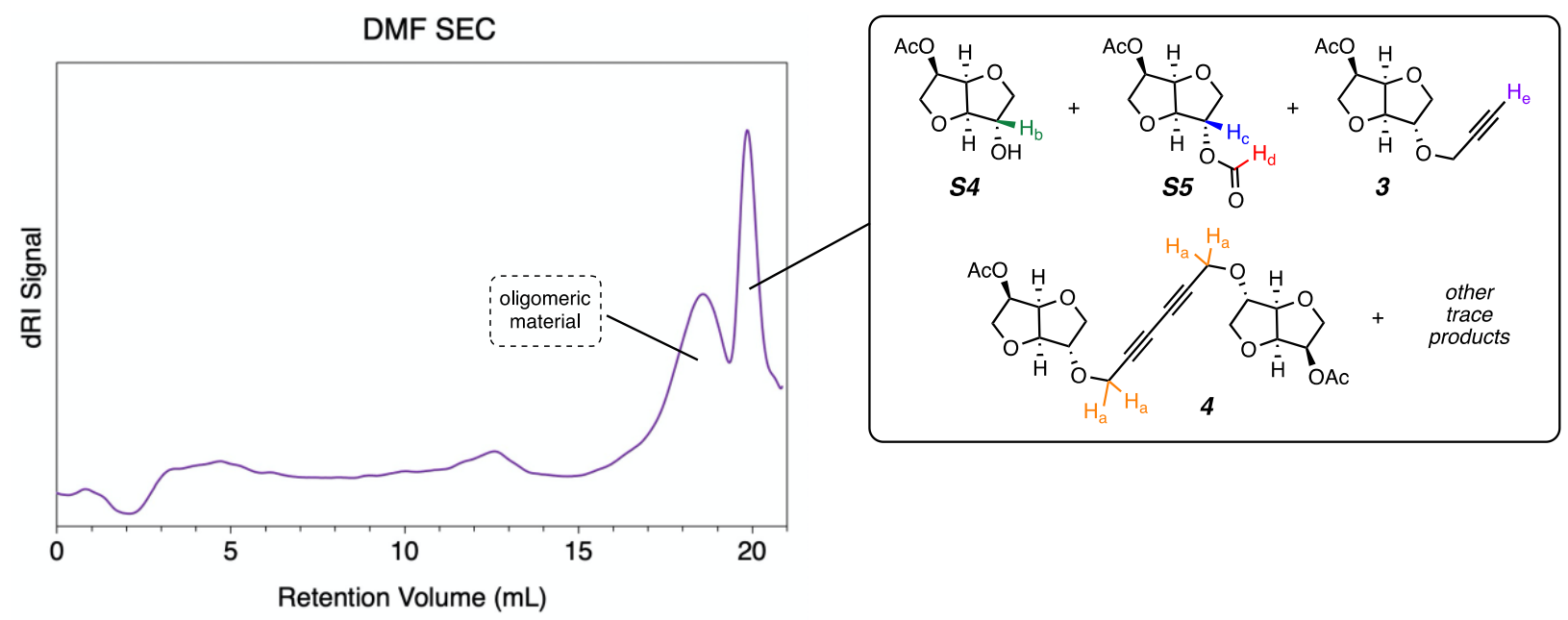

Figure S24. SEC analysis (DMF mobile phase) of the oxidation products obtained from heating 4.

\section{References for Supporting Information}

${ }^{1}$ Biomacromolecules 2010, 11, 2797-2803

${ }^{2}$ Green Chem. 2014, 16, 3810-3818

${ }^{3}$ Biomacromolecules 2012, 13, 4138-4145

${ }^{4}$ J. Am. Chem. Soc. 2019, 13, 5107-5111

\section{Copies of ${ }^{1} \mathrm{H}$ and ${ }^{13} \mathrm{C}$ NMR Spectra}




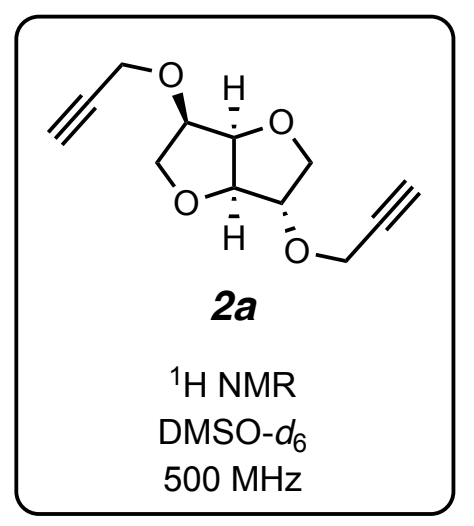

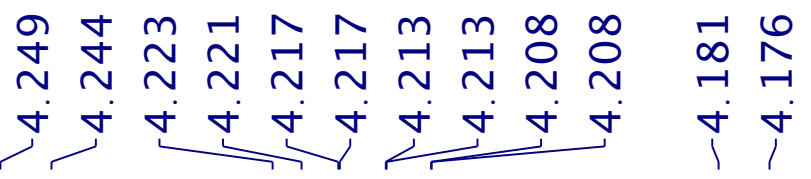

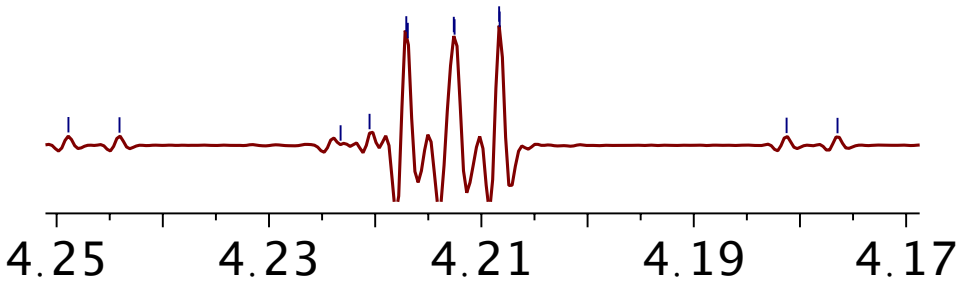

Nิ๊

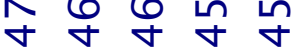

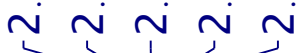
나의 ○ $\forall \dot{r} \dot{\sim} \dot{m} \dot{m} \dot{m}$
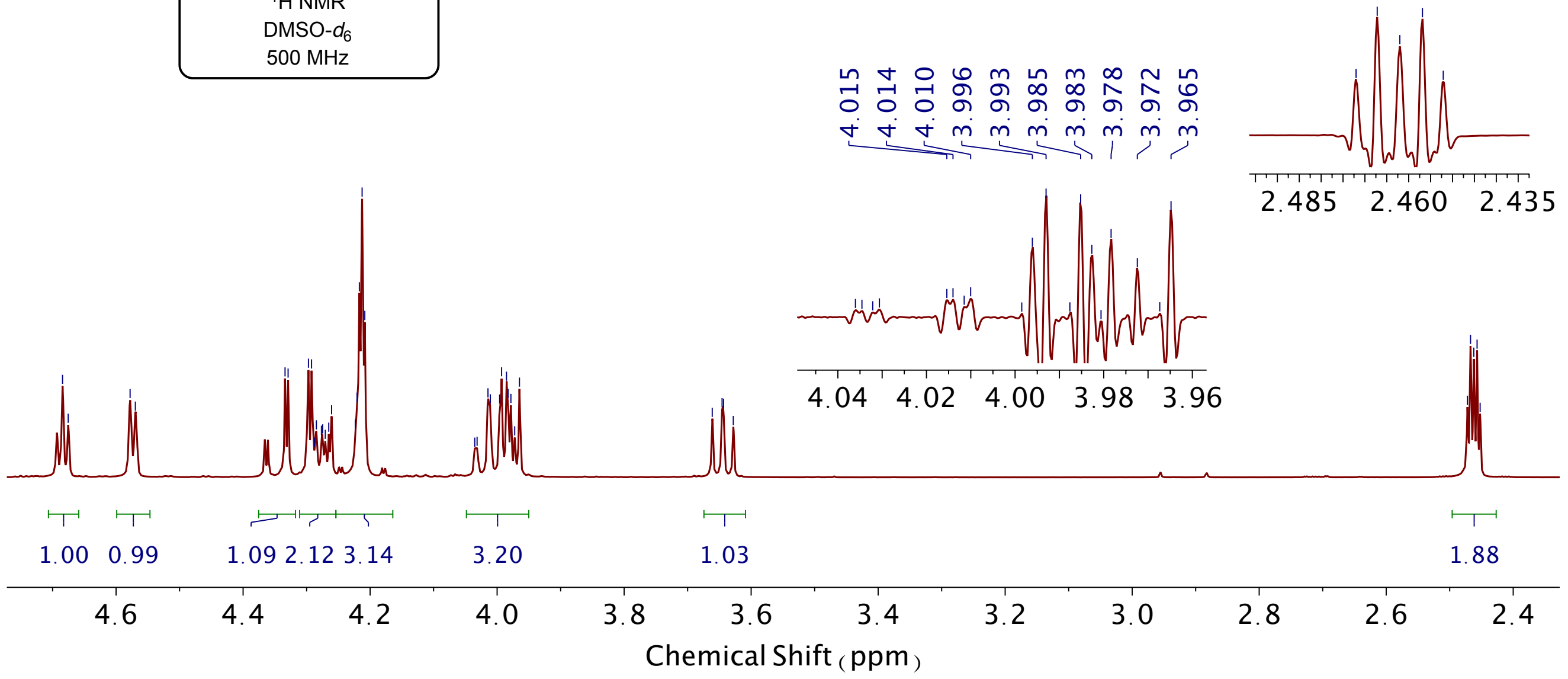


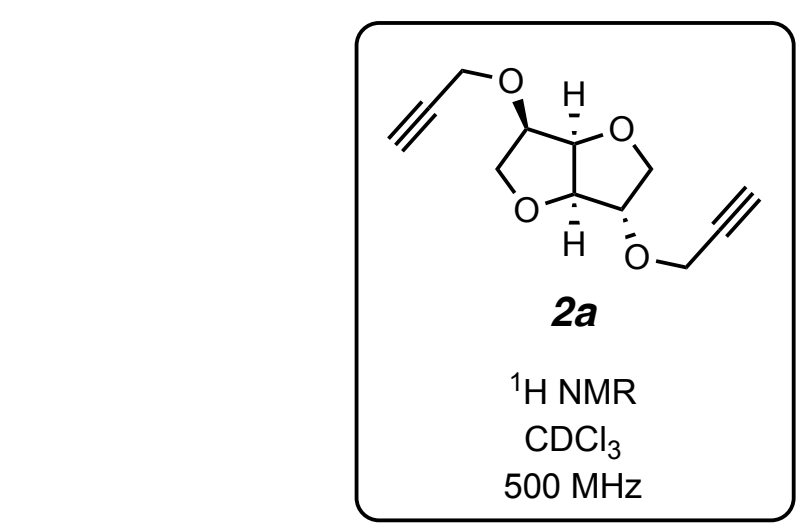

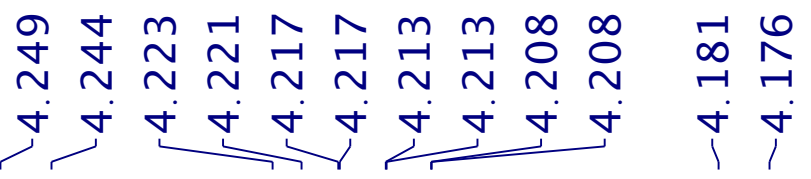

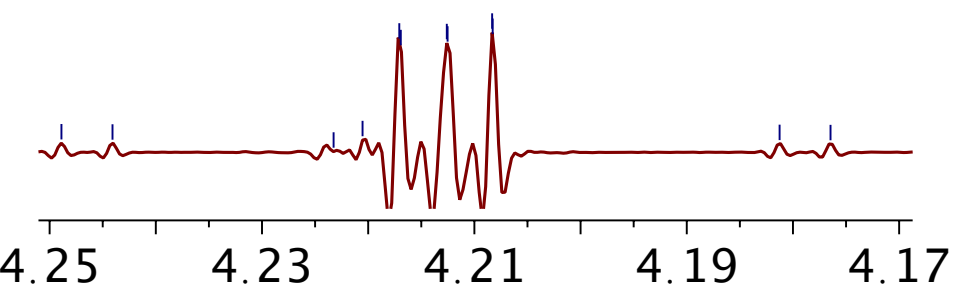

กิู

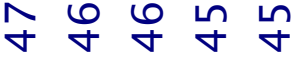

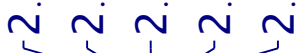
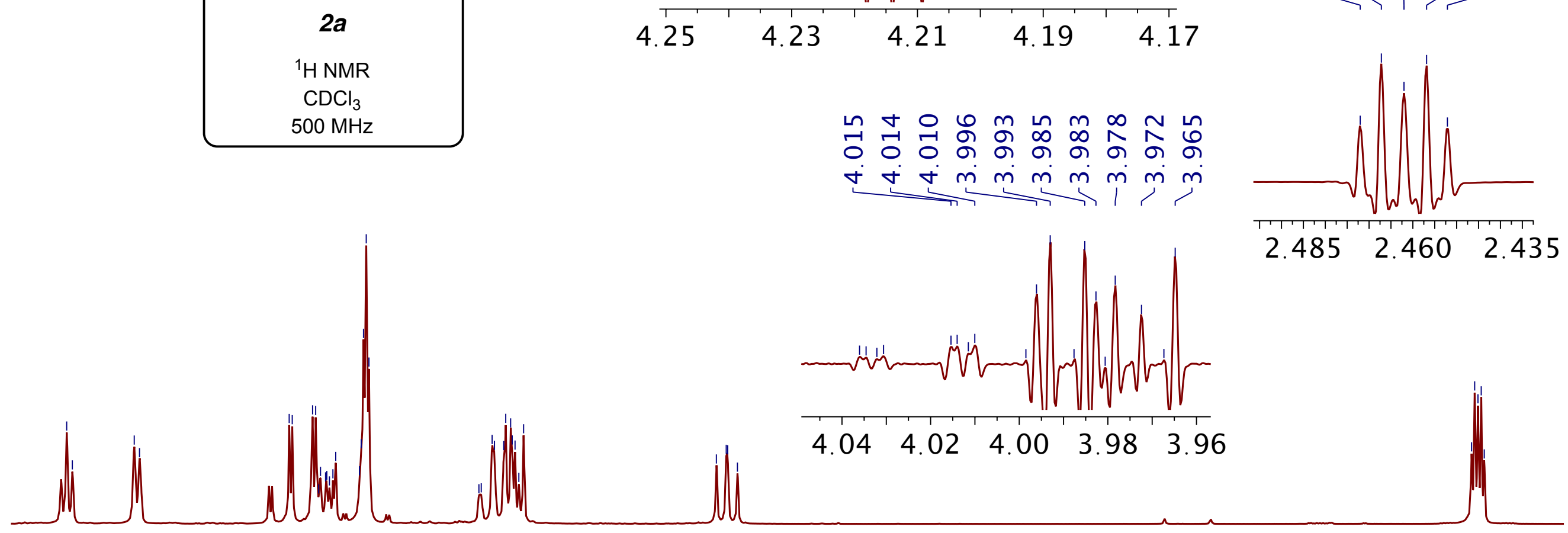

$\mapsto \mapsto$

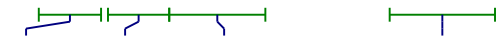

1.03

1.88

4. 6

4. 4

4. 2

4. 0

3.8

3.6

3.4

3.2

3. 0

2.8

2.6

2.4 


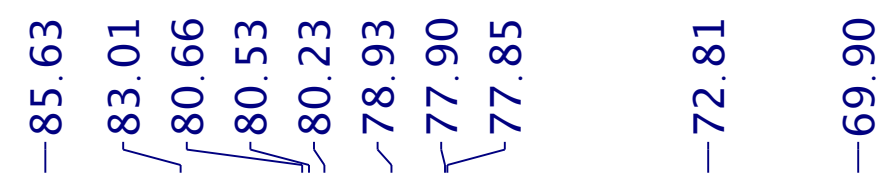

$\stackrel{m}{m} \stackrel{+}{r}$

No

กิ ถก

$1\}$

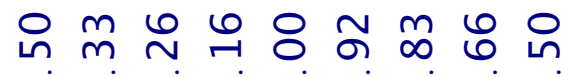
○
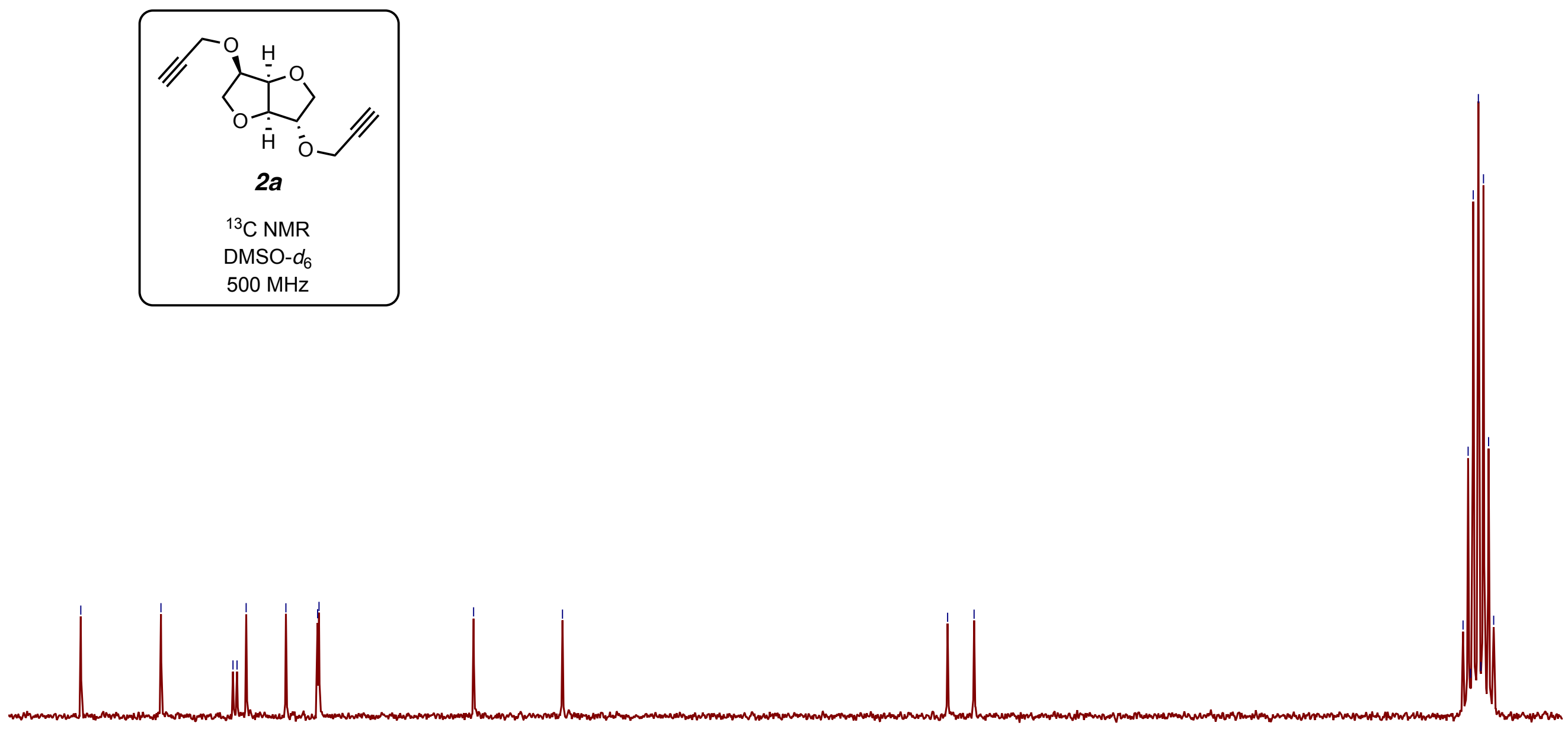


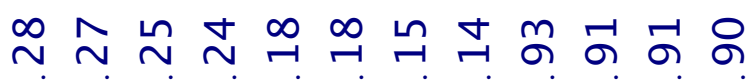
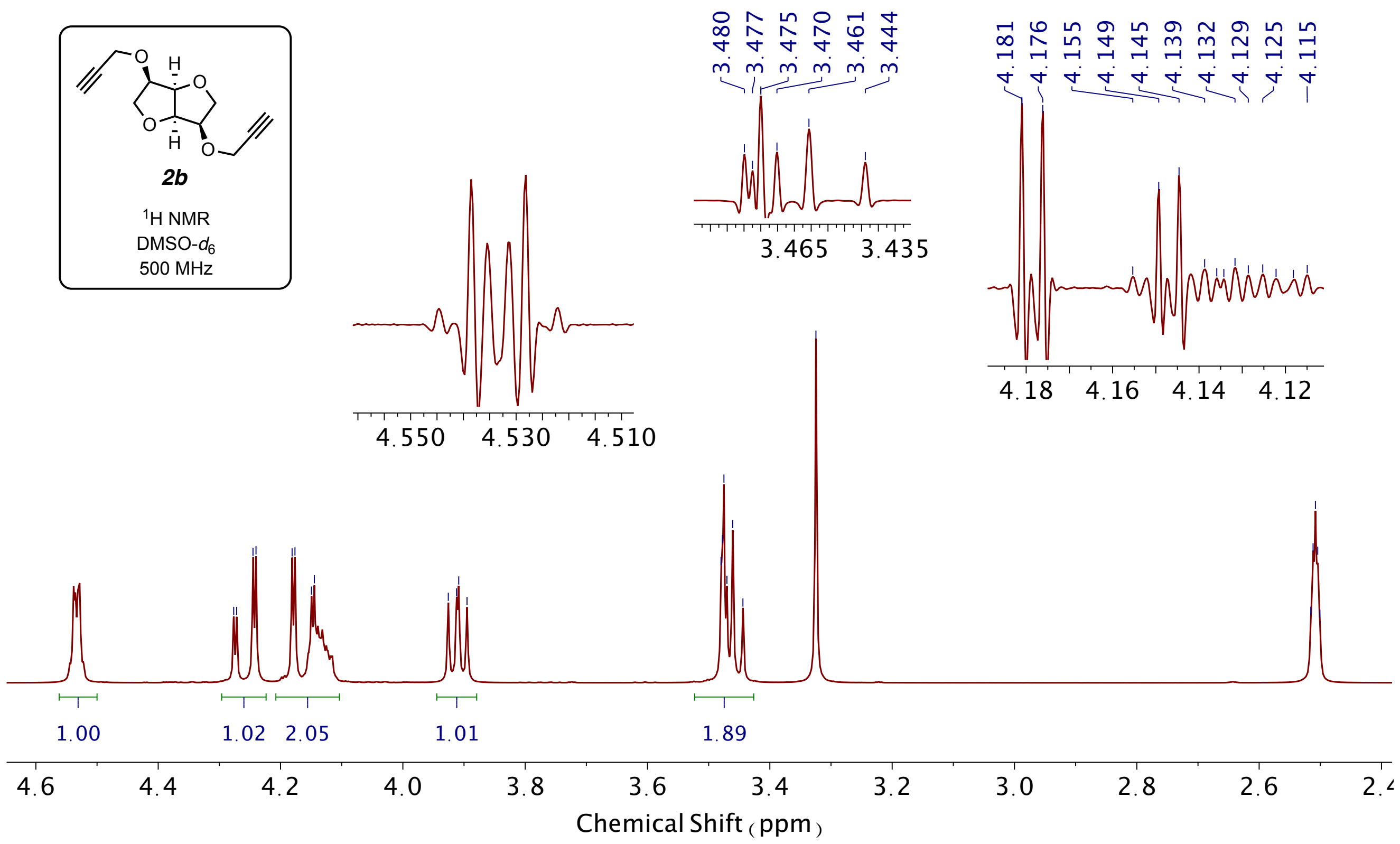

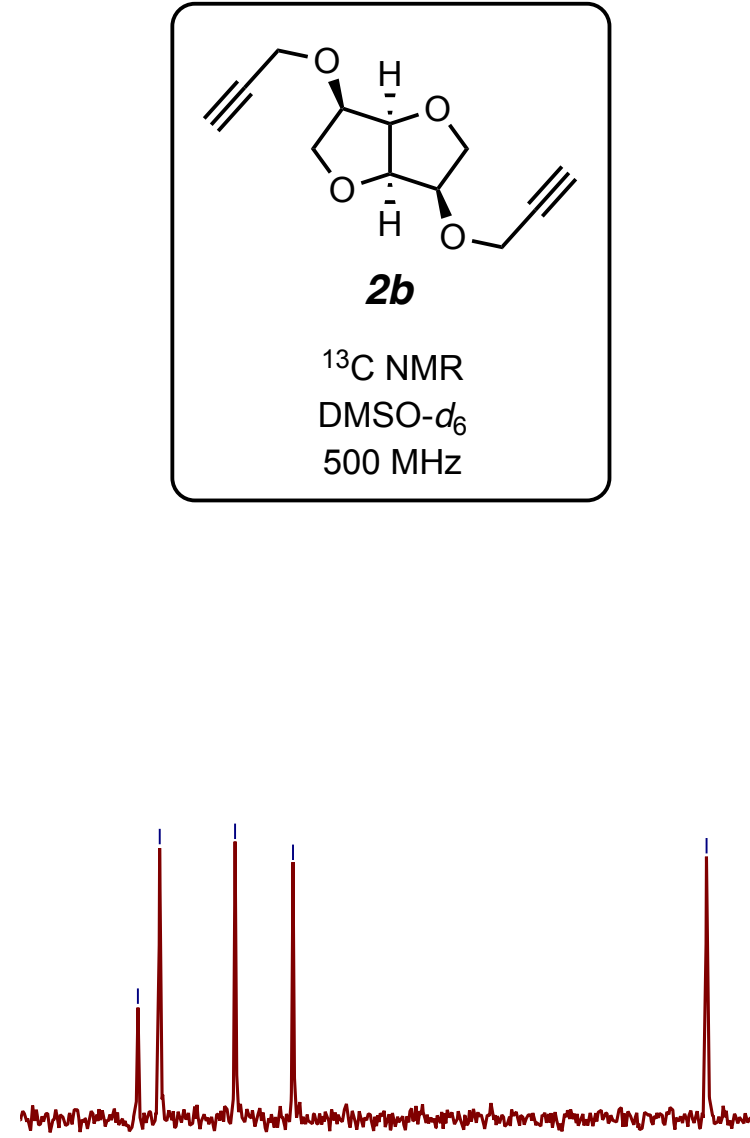

$$
\text { กิ }
$$



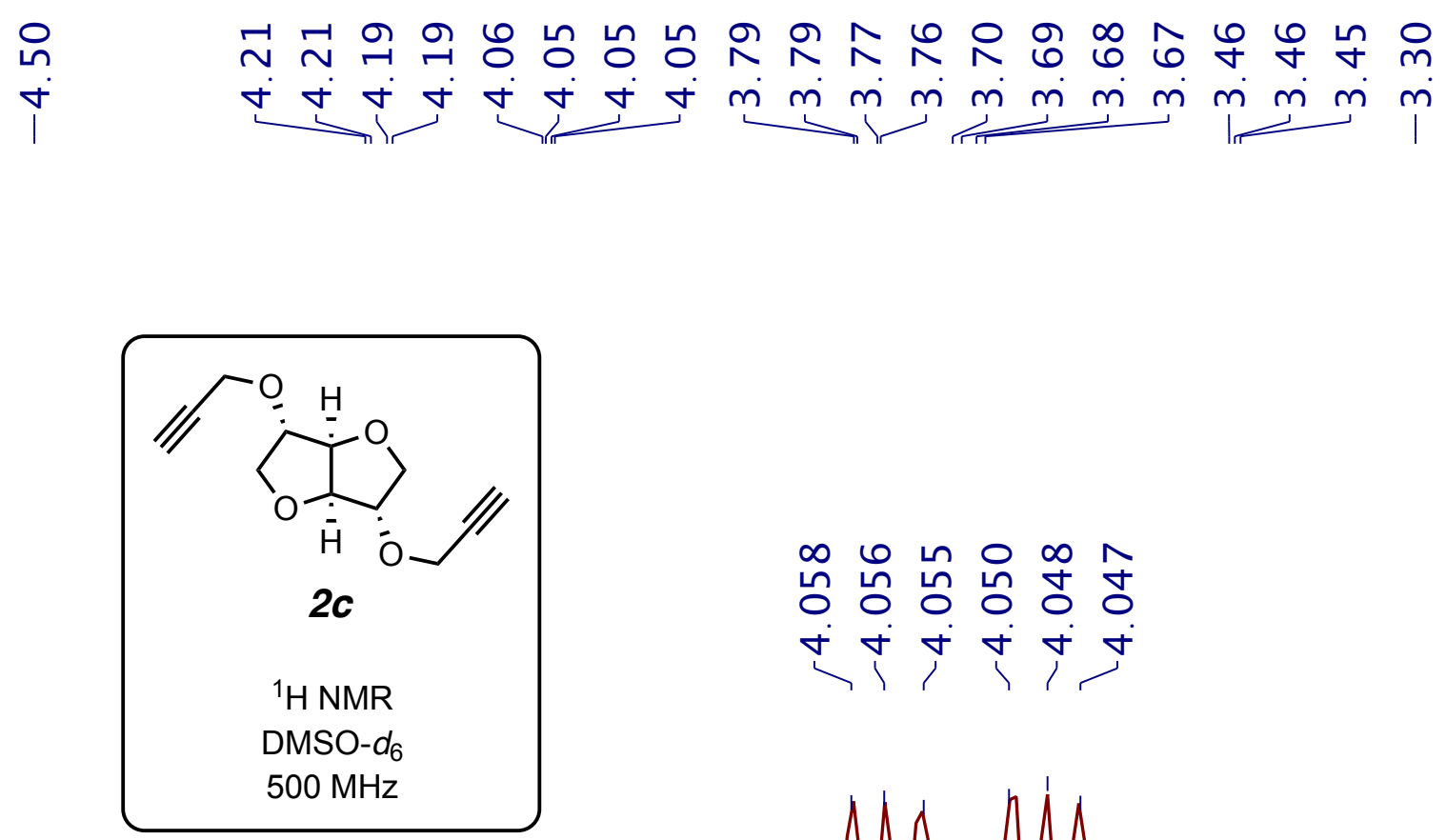

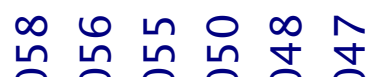

ก

Aij,
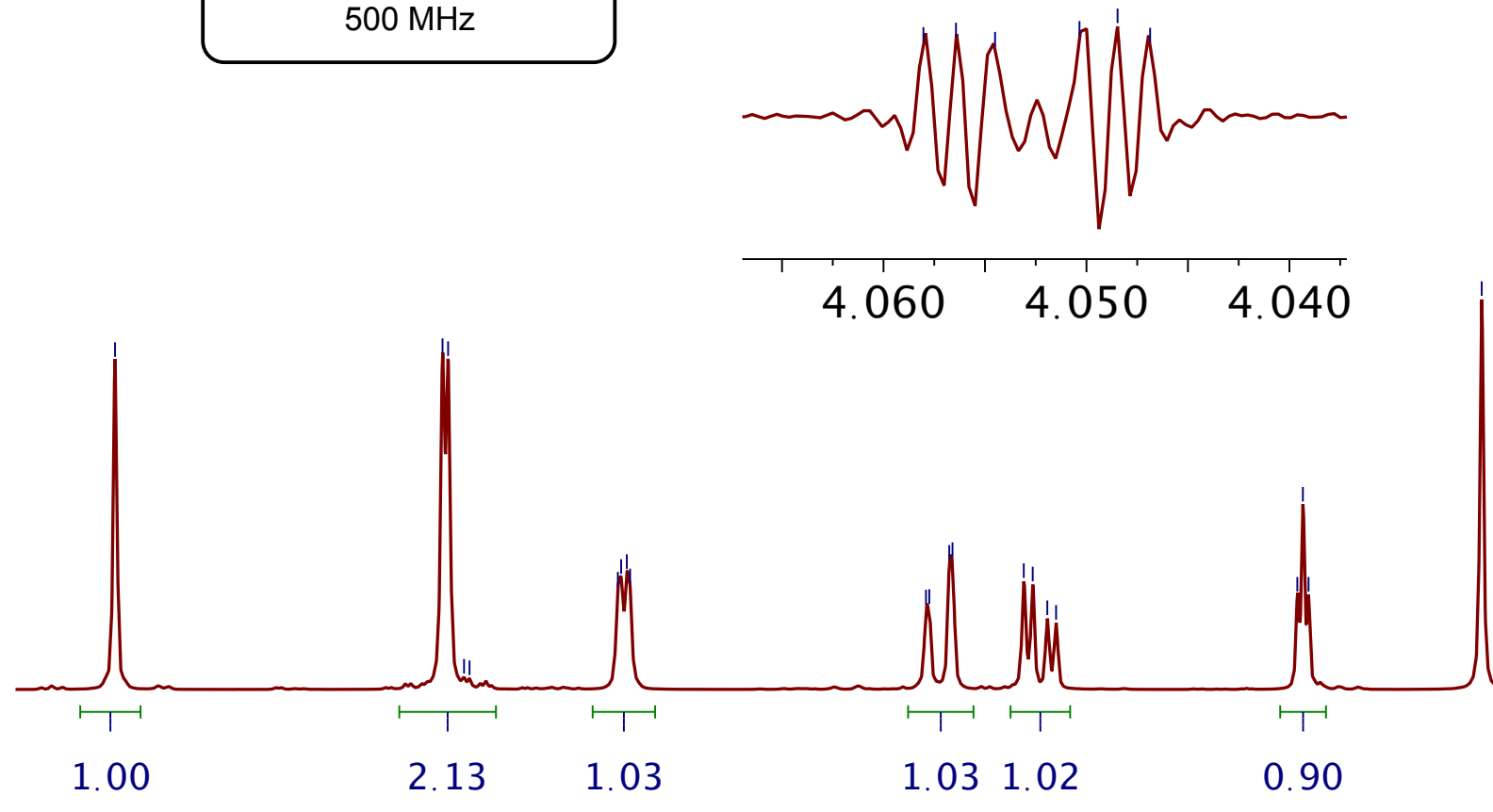

$\begin{array}{lllllllllllllllllllllll}4.5 & 4.4 & 4.3 & 4.2 & 4.1 & 4.0 & 3.9 & 3.8 & 3.7 & 3.6 & 3.5 & 3.4 & 3.3 & 3.2 & 3.1 & 3.0 & 2.9 & 2.8 & 2.7 & 2.6 & 2.5\end{array}$ Chemical Shift $(\mathrm{ppm})$ 

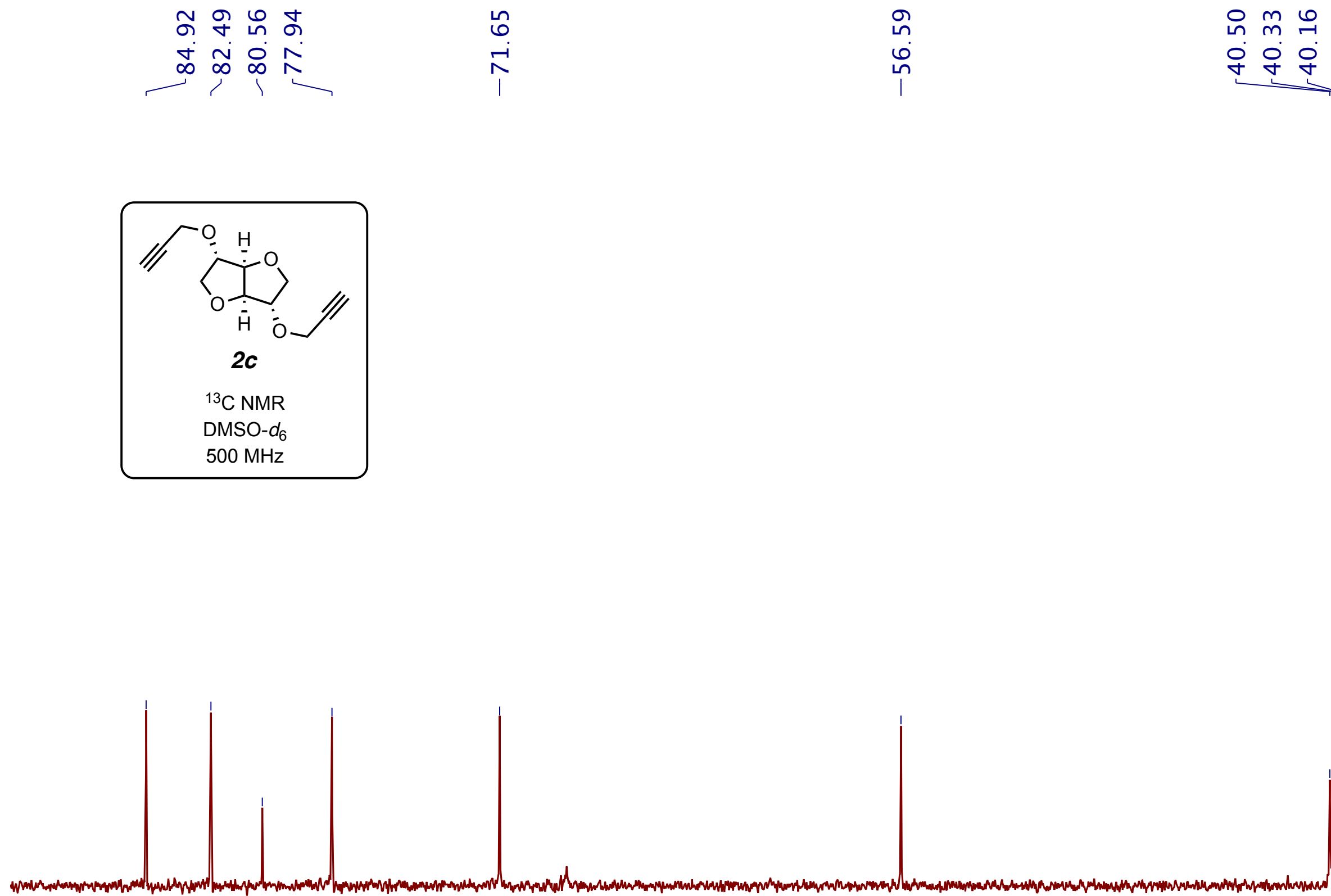


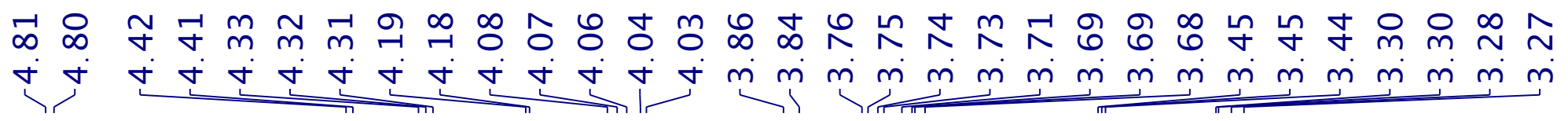
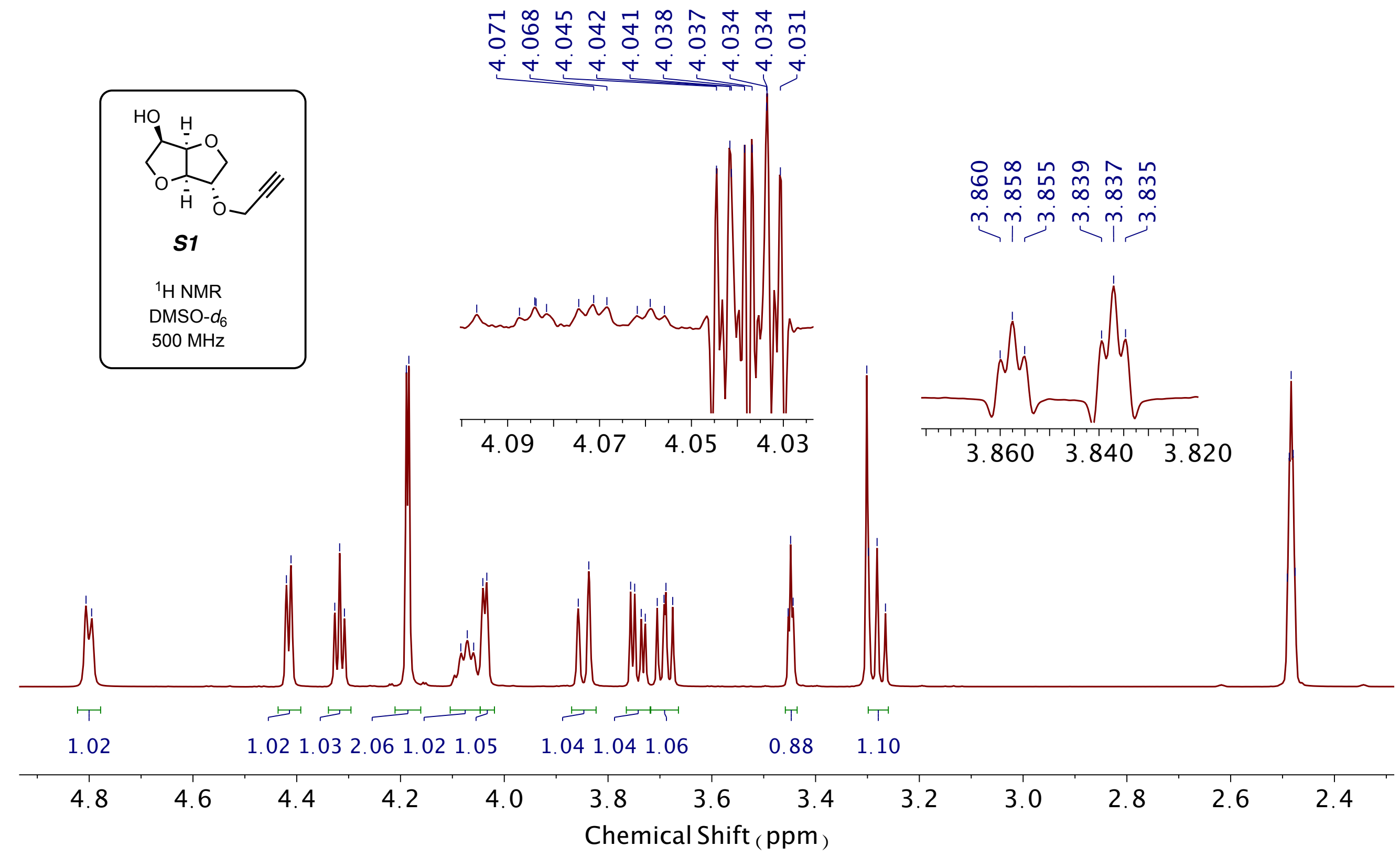


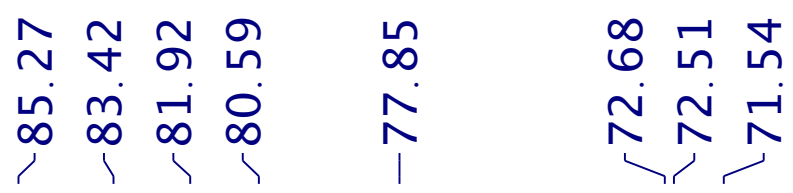
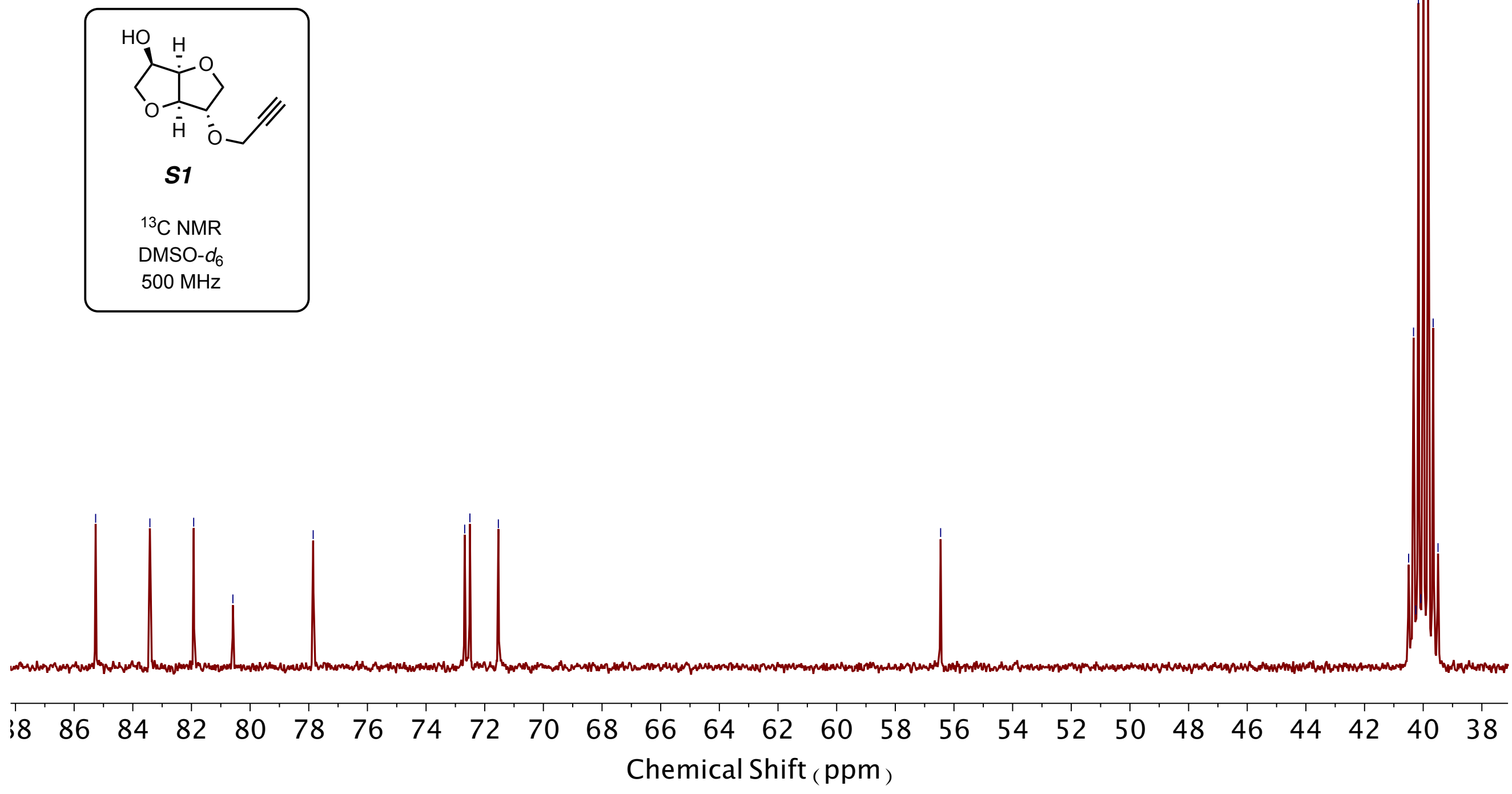


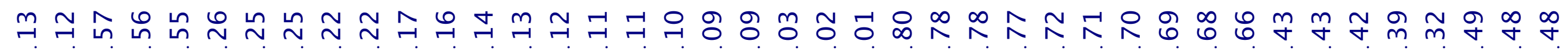

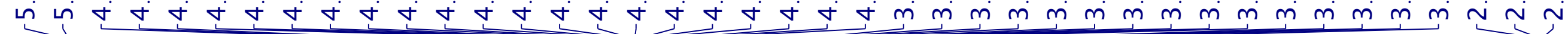

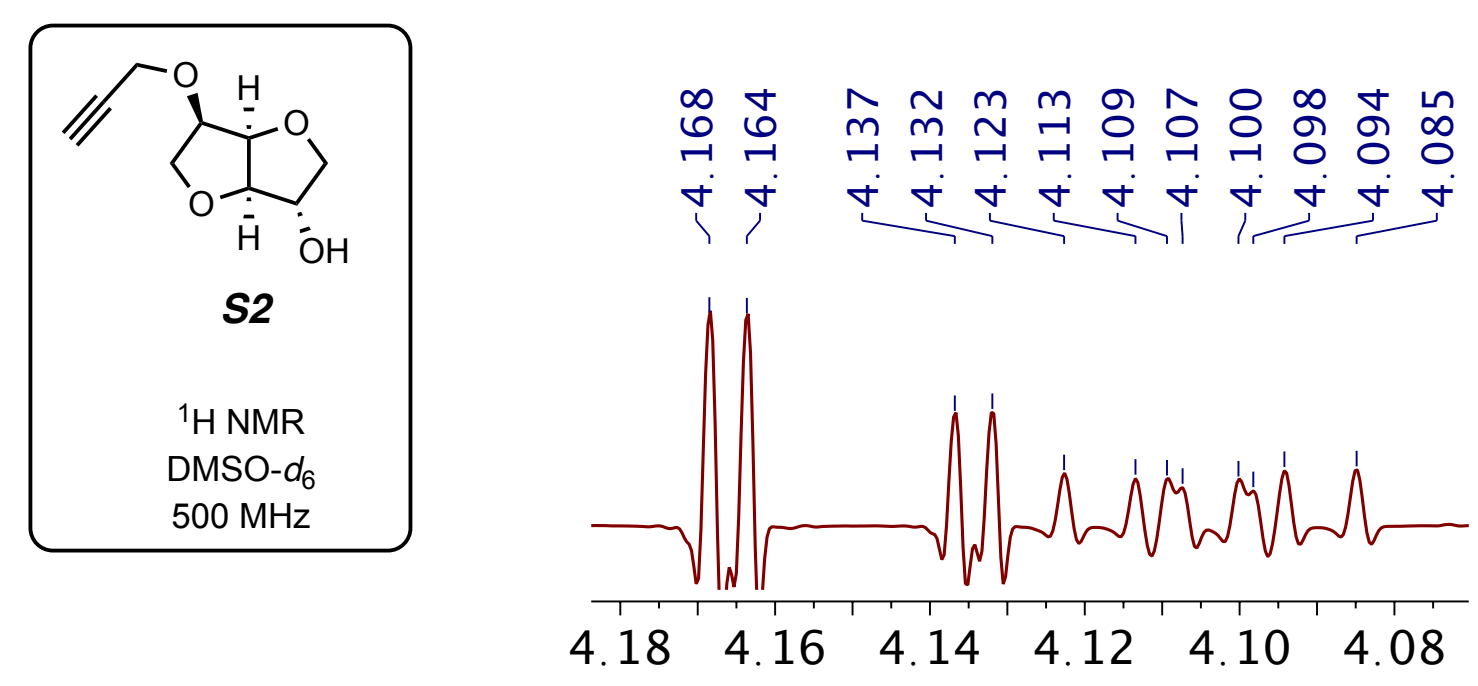

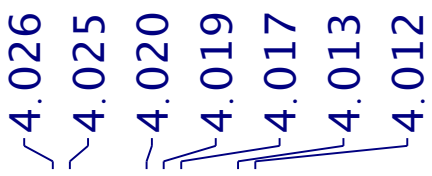
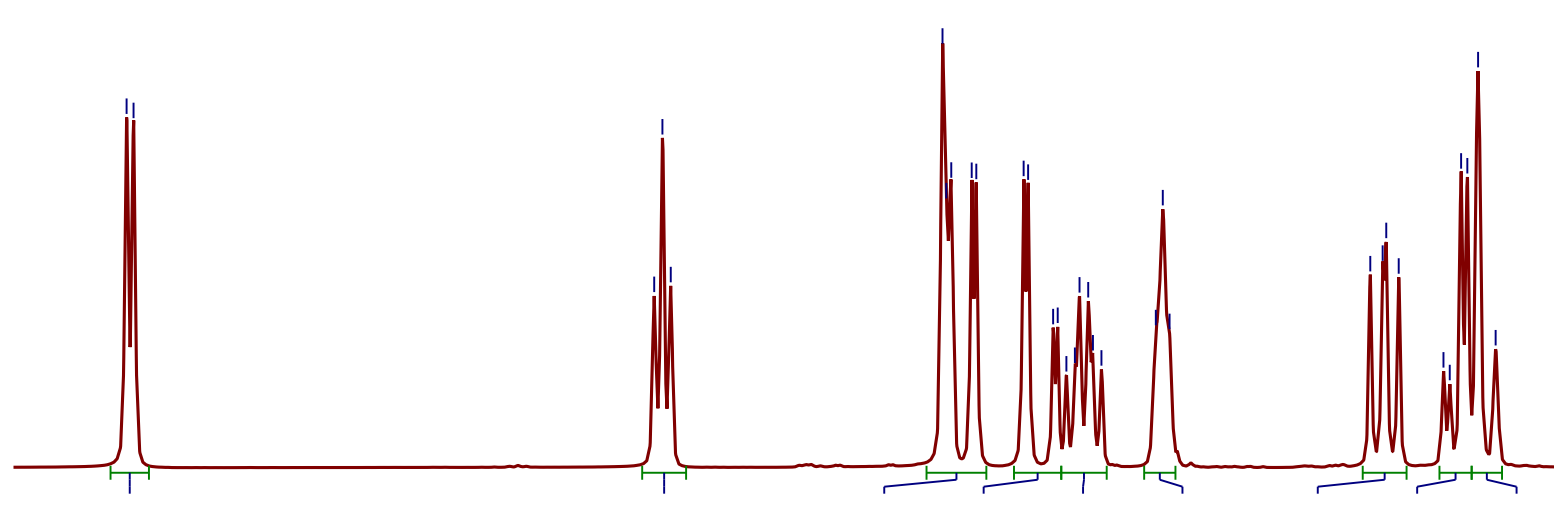

0.99

$2.011 .001 .001 .00 \quad 1.001 .021 .00$

0.851 .01

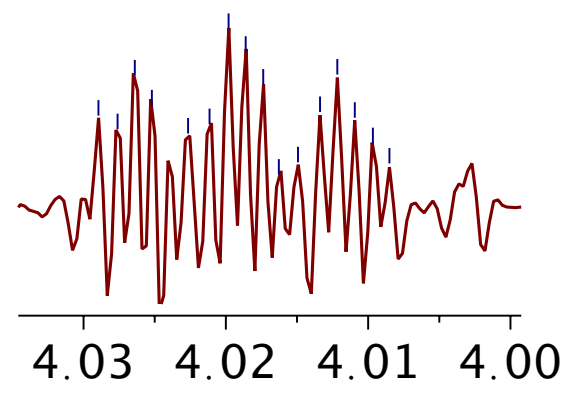

$5.2 \quad 5.0$

4.8

4.6

4.4

4.2

$\begin{array}{ll}4.0 & 3.8\end{array}$

3. 6

3.4

3.2

3.0

2.8

2.6

Chemical Shift (ppm) 

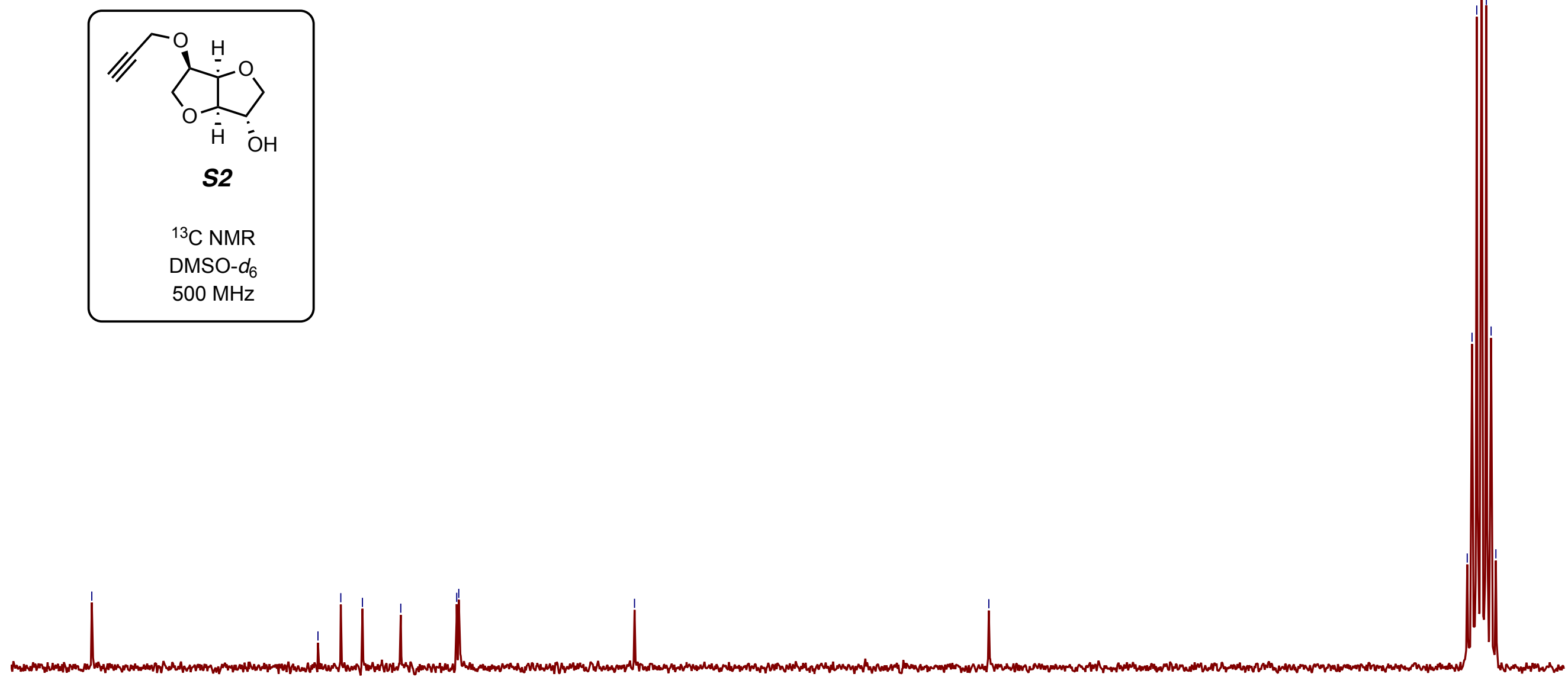

$\begin{array}{lllllllllllllllllllllllllll}90 & 88 & 86 & 84 & 82 & 80 & 78 & 76 & 74 & 72 & 70 & 68 & 66 & 64 & 62 & 60 & 58 & 56 & 54 & 52 & 50 & 48 & 46 & 44 & 42 & 40 & 38\end{array}$ Chemical Shift ( $\mathrm{ppm}$ ) 
$\stackrel{\bullet}{\sim}$

î

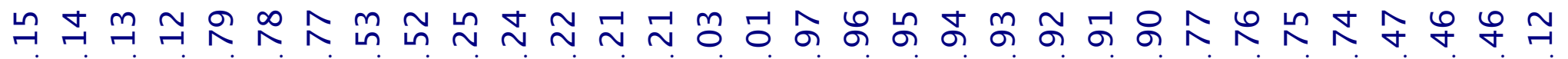

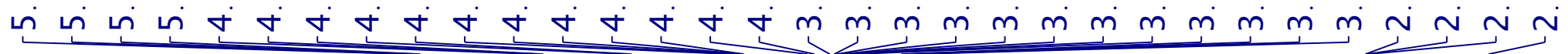

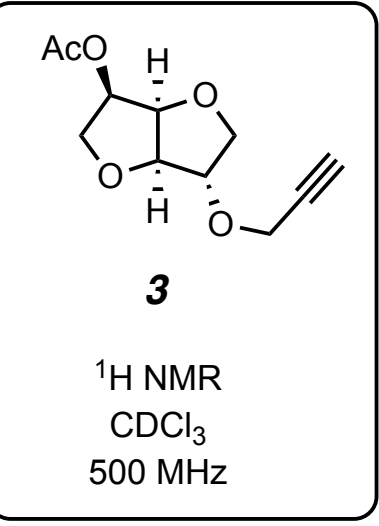

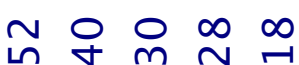
ก

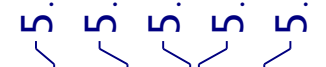

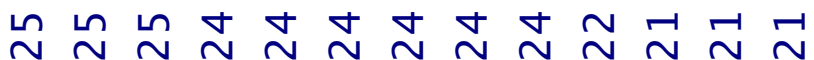

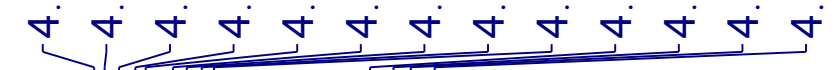
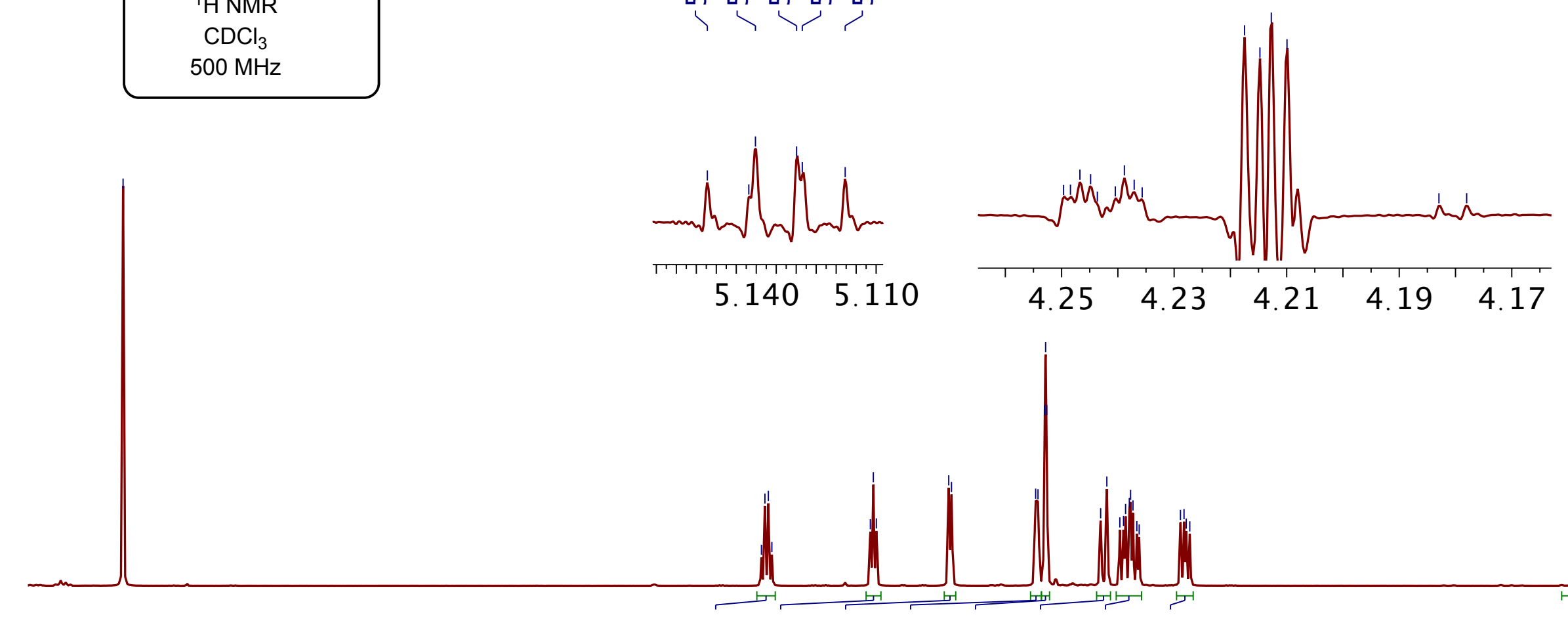

1.001 .001 .001 .061 .971 .032 .091 .03

0.91

3.09

7.5

7.0

6.5

6.0

5.5

4.5

4. 0

3.5

3. 0

2.5

2.0

Chemical Shift (ppm) 

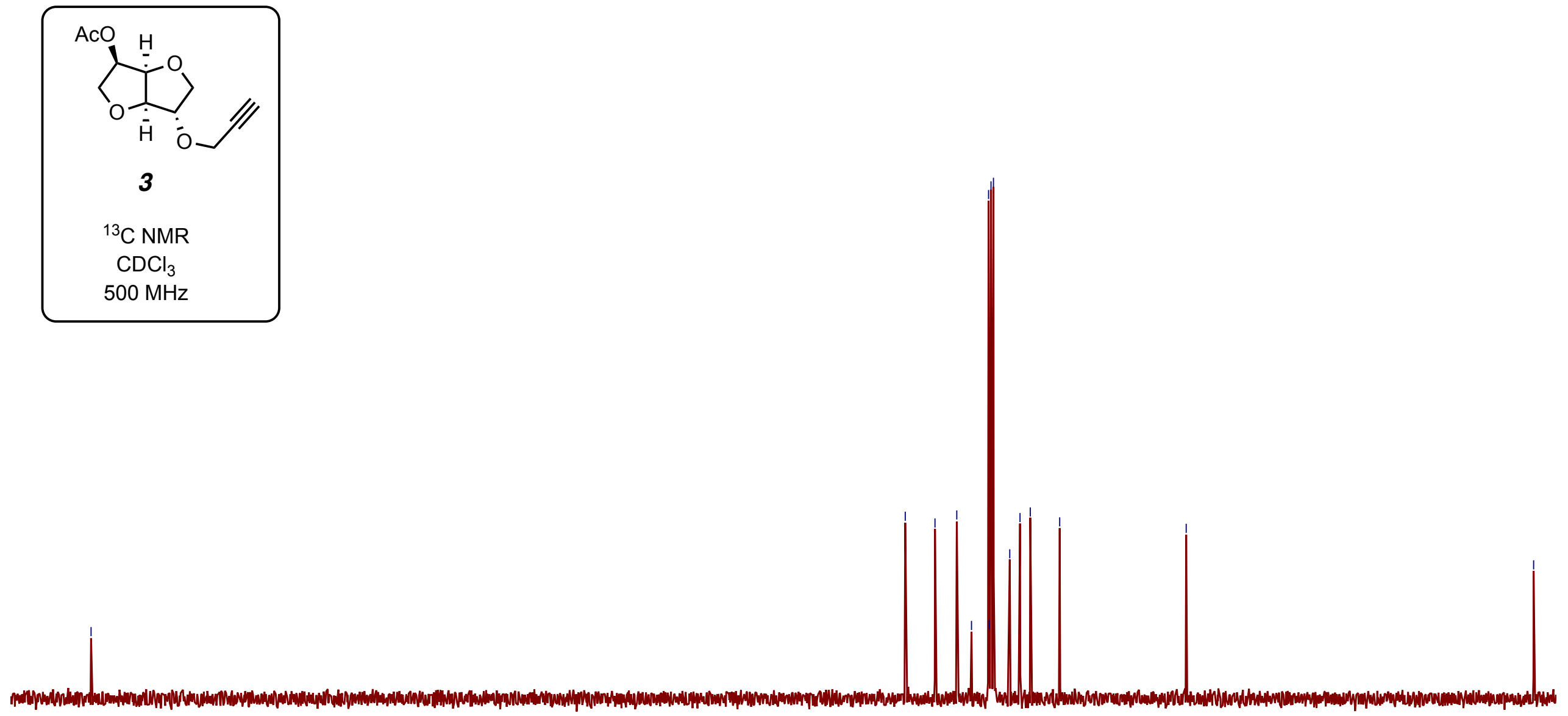

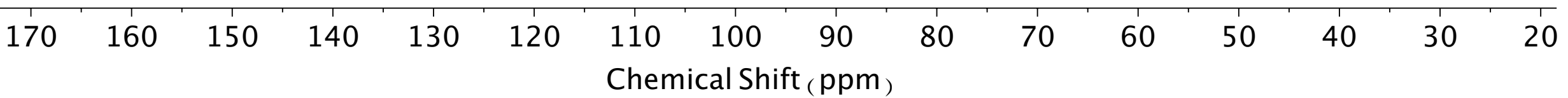



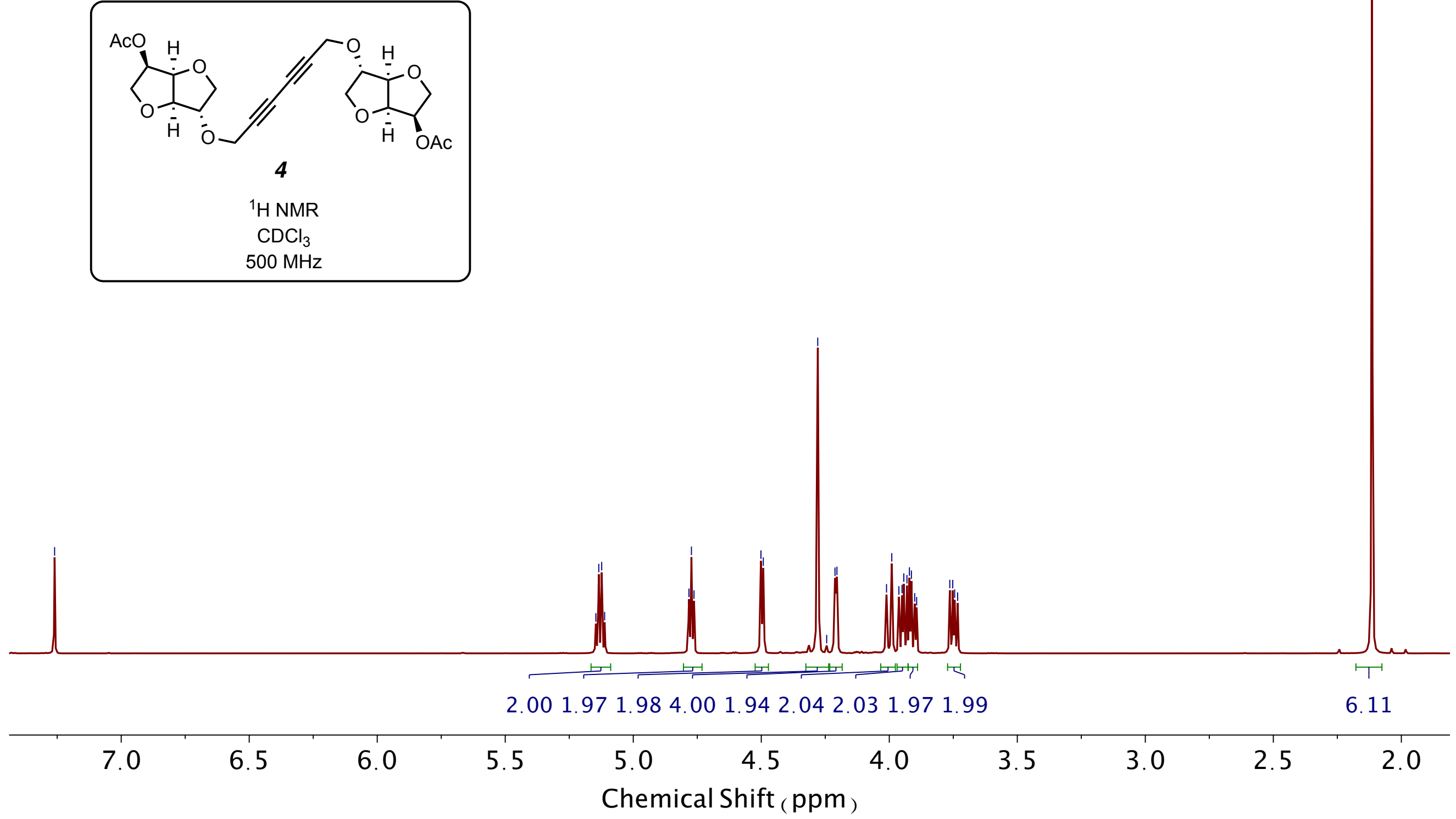
$\infty$
$m$

$i$
$i$
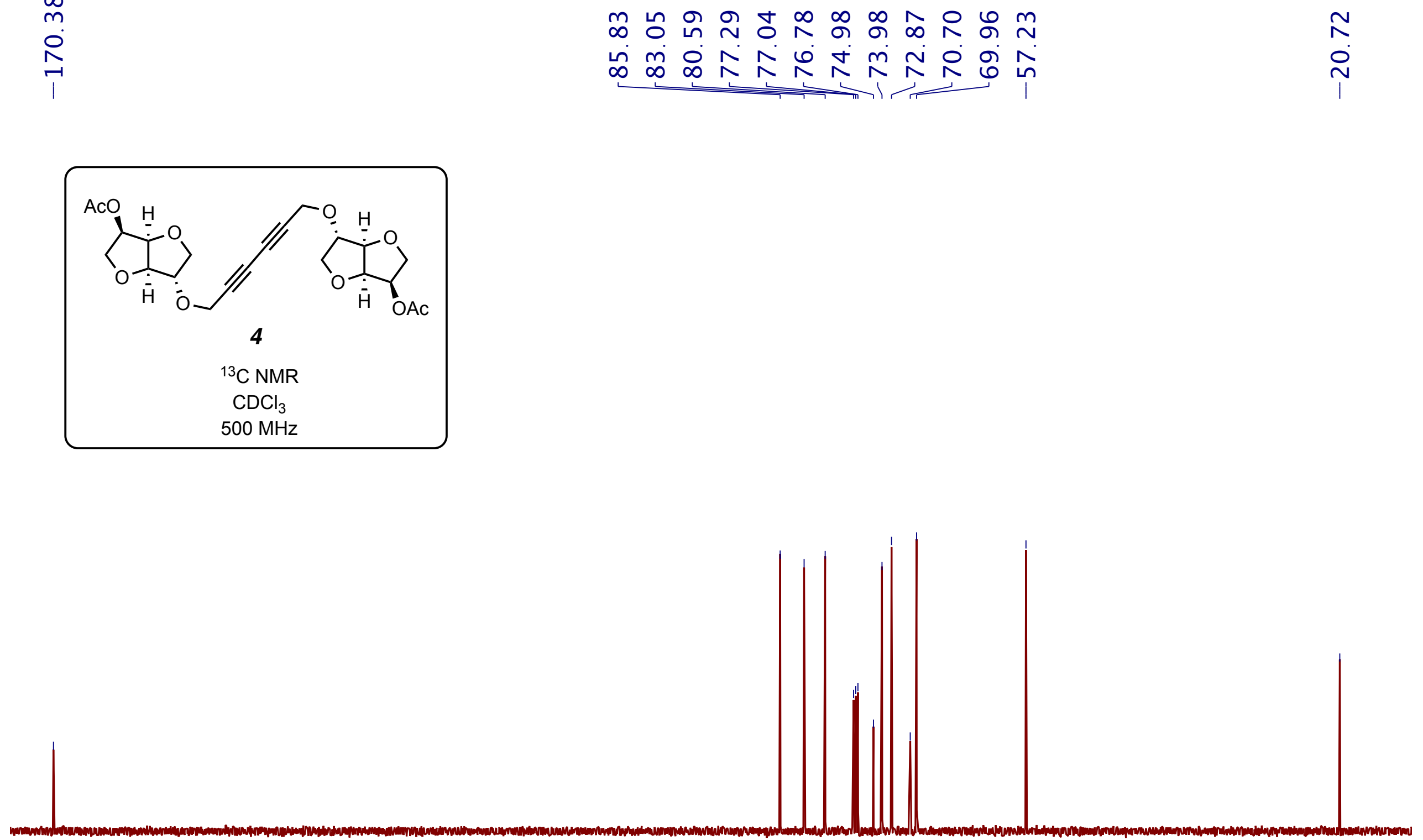

$\begin{array}{lllllllllllllll}170 & 160 & 150 & 140 & 130 & 120 & \begin{array}{c}110 \\ \text { Chemical Shift }(\mathrm{ppm})\end{array} & \begin{array}{c}100 \\ 90\end{array} & 80 & 70 & 60 & 50 & 40 & 30 & 20\end{array}$




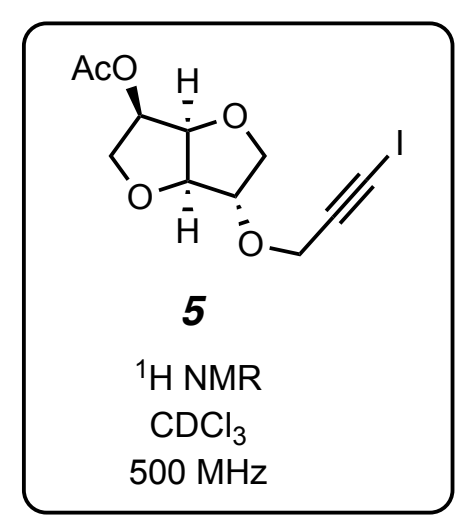

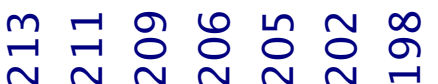

r
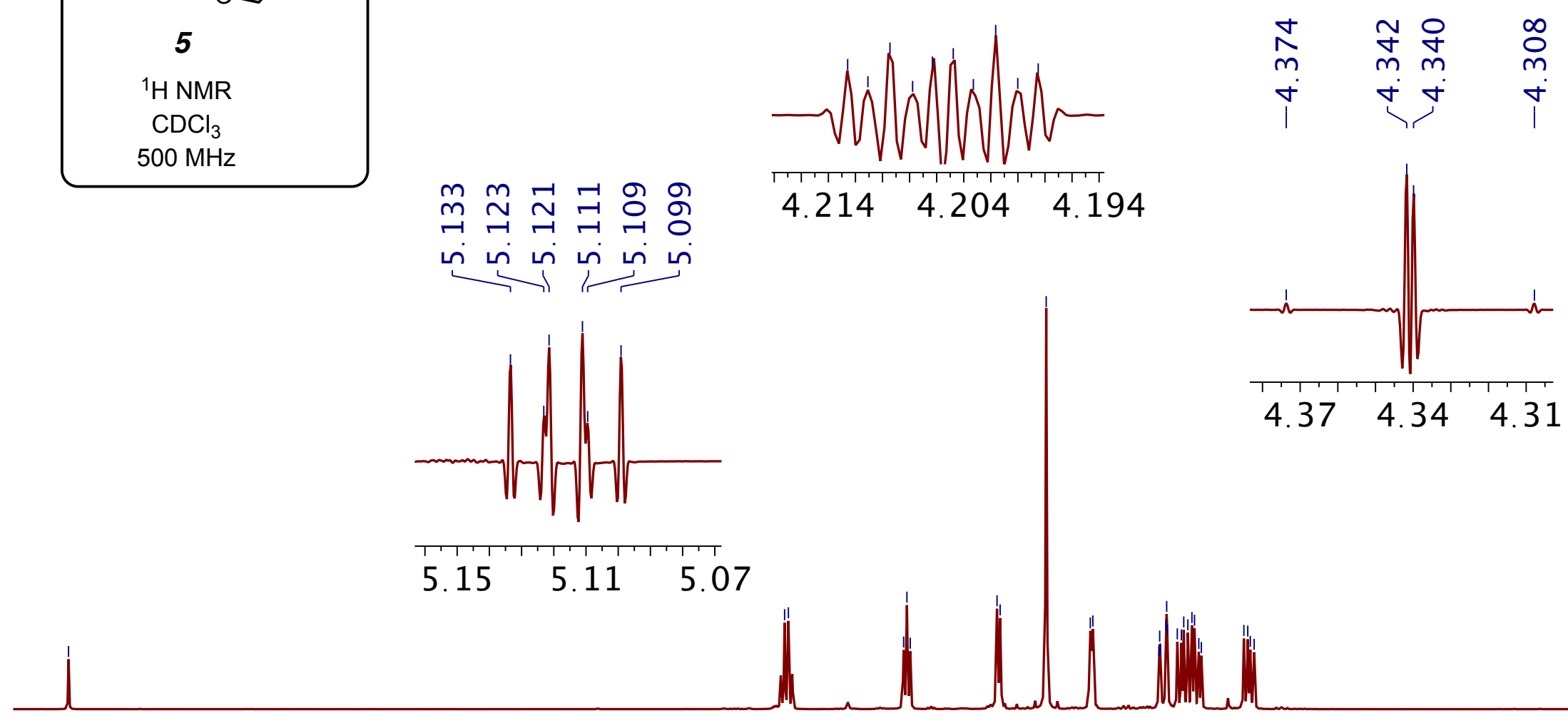

\section{$\begin{array}{lll}5.15 & 5.11 & 5.07\end{array}$}

0.980 .970 .992 .020 .971 .041 .051 .001 .00

4.0

3.5



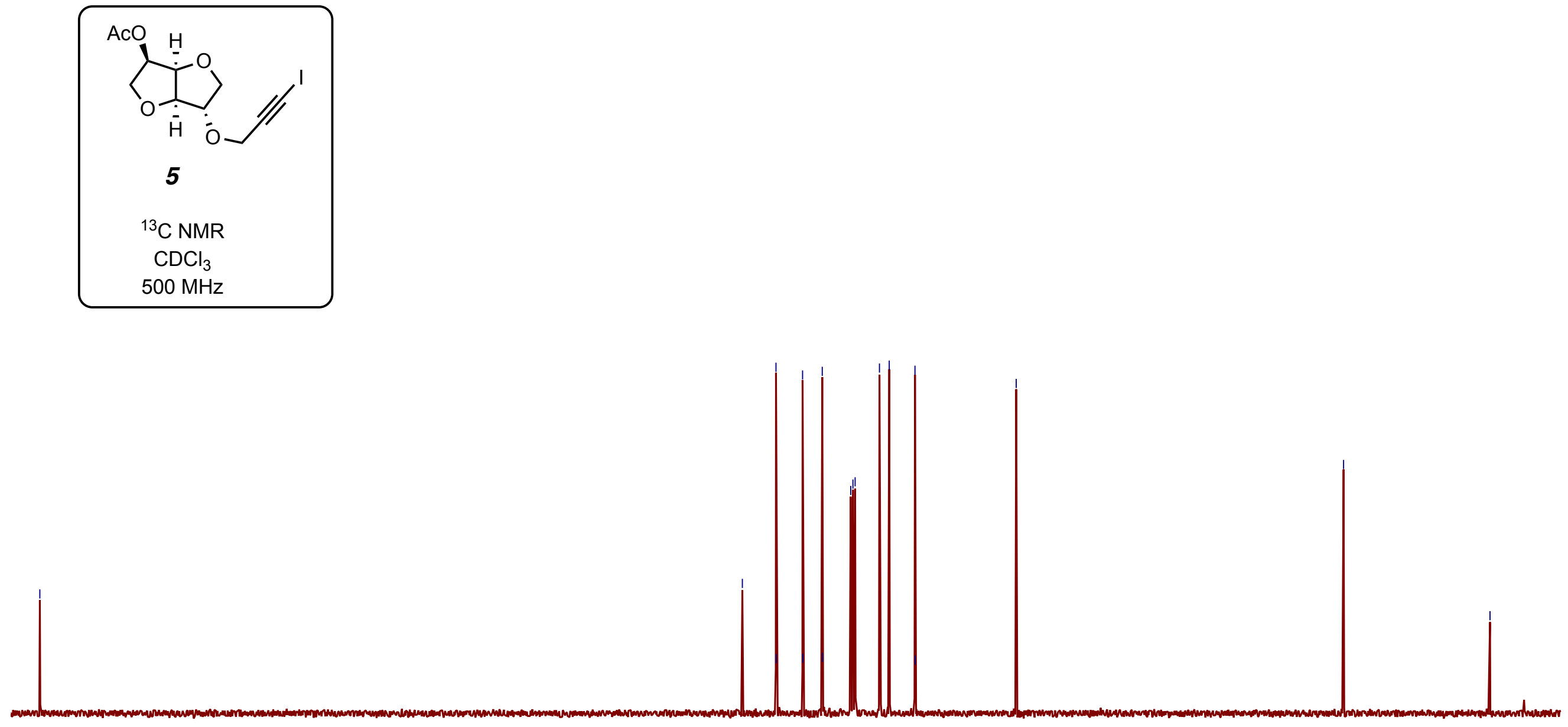

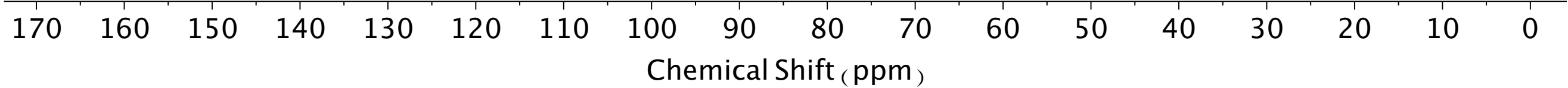



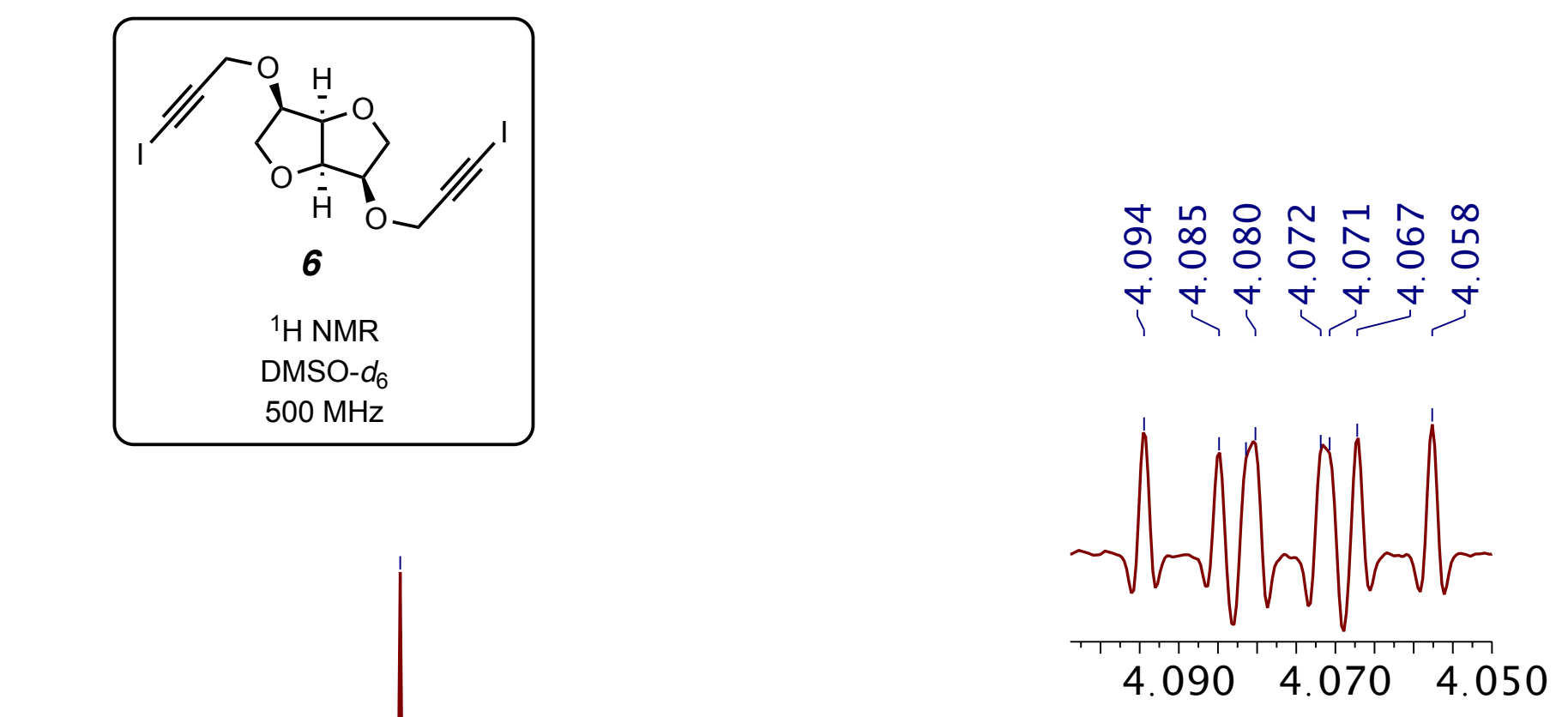

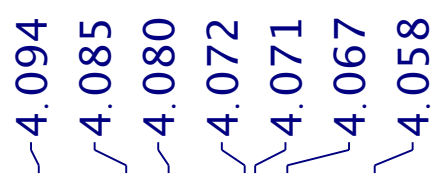
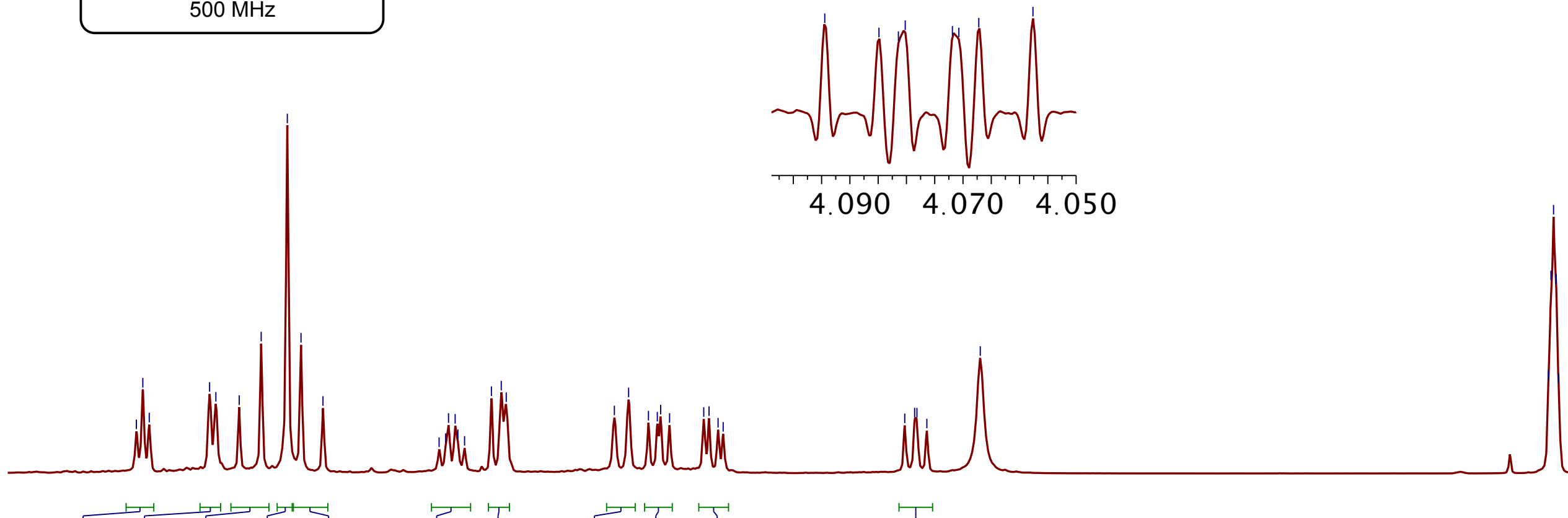

$\begin{array}{llllll}1.001 .03 & 1.14 & 1.98 & 1.14 & 1.071 .36 \quad 1.051 .061 .03 & 0.99\end{array}$

\begin{tabular}{|c|c|c|c|c|c|c|c|c|c|c|}
\hline 4.6 & 4.4 & 4.2 & 4.0 & 3.8 & 3.6 & 3.4 & 3.2 & 3.0 & 2.8 & 2.6 \\
\hline & & & & & nical & ppm) & & & & \\
\hline
\end{tabular}




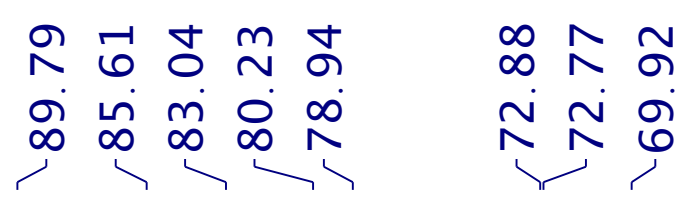

$$
\begin{array}{ll}
\sim & 0 \\
0 & 0 \\
\infty & \infty \\
\text { n? } & 0
\end{array}
$$

이 $m m$ ri
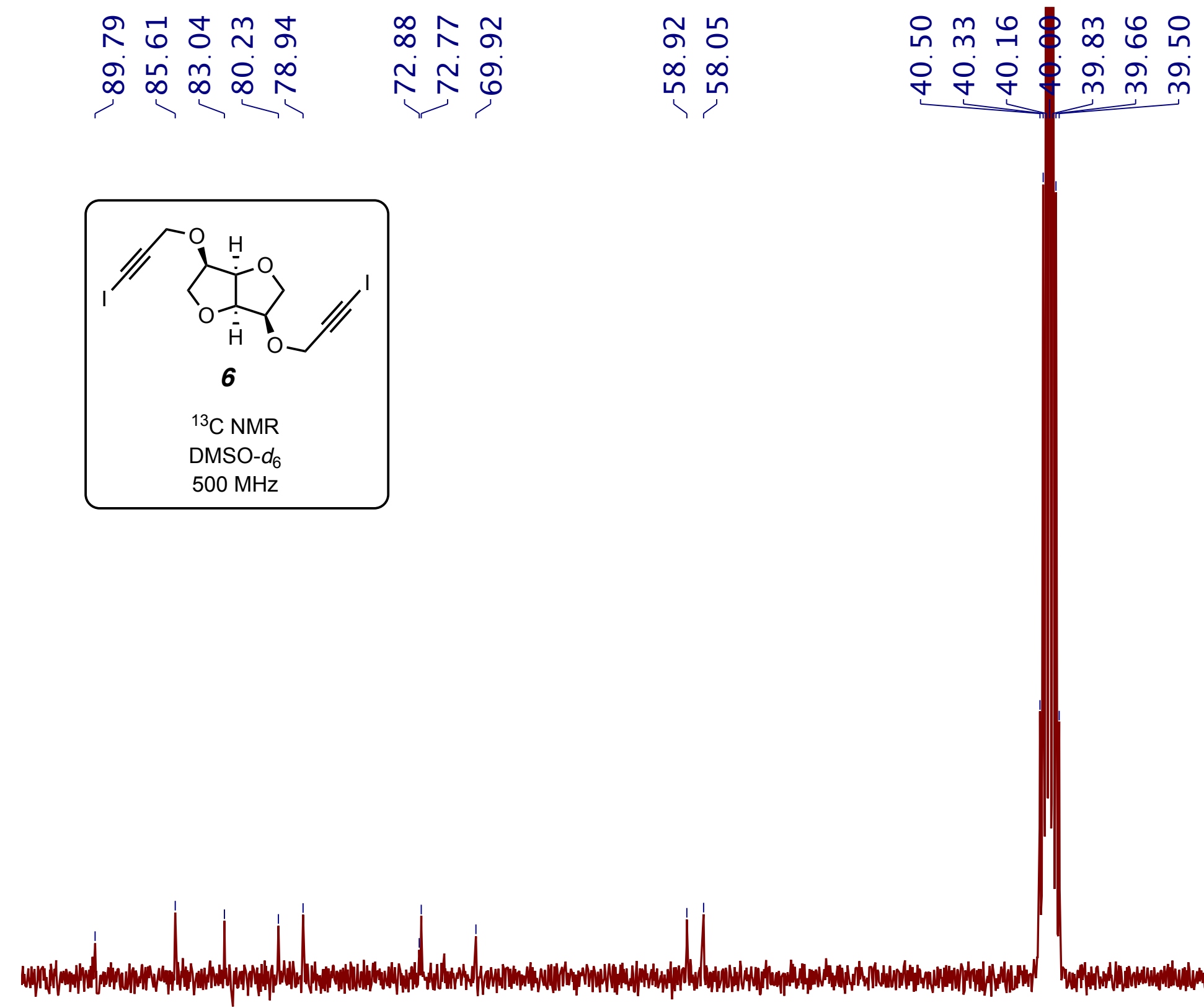

$\underbrace{\sim}$

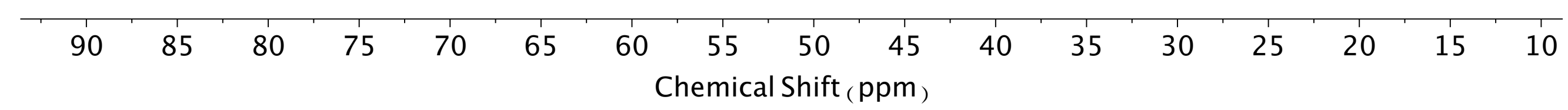



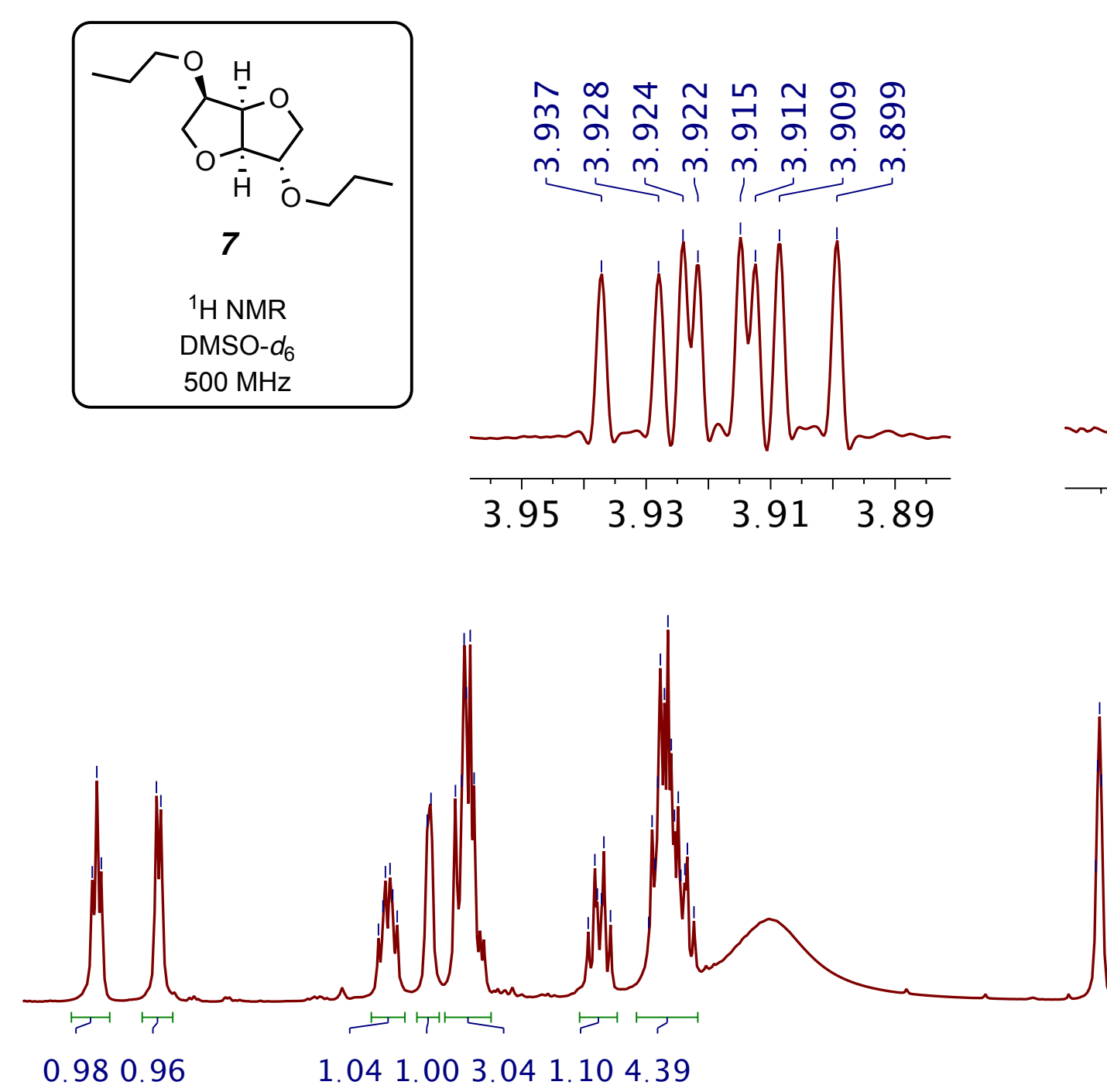

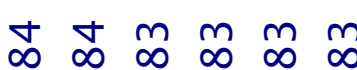

$m \dot{m} \dot{m} \dot{m} \dot{m} \dot{m}$

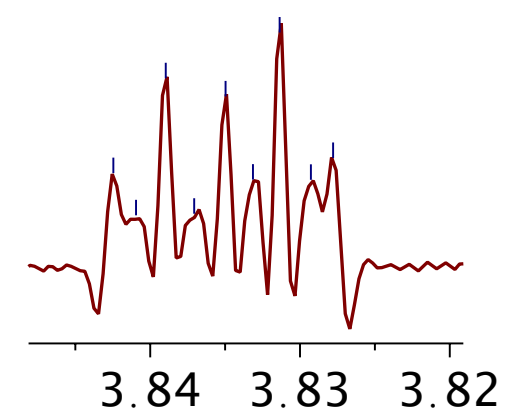

$F \hat{\infty} \approx \approx N$

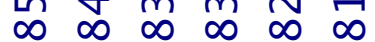

ن门i i i i

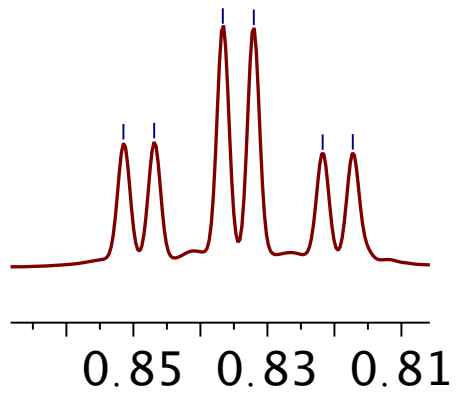

$3.84 \quad 3.83 \quad 3.82$

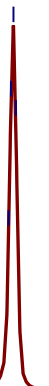

3.87

5.72

\begin{tabular}{lllllllllllllllllllllllllll}
\hline 4.6 & 4.4 & 4.2 & 4.0 & 3.8 & 3.6 & 3.4 & 3.2 & 3.0 & 2.8 & 2.6 & 2.4 & 2.2 & 2.0 & 1.8 & 1.6 & 1.4 & 1.2 & 1.0 & 0.8 & 0.6
\end{tabular} Chemical Shift ( $\mathrm{ppm}$ ) 


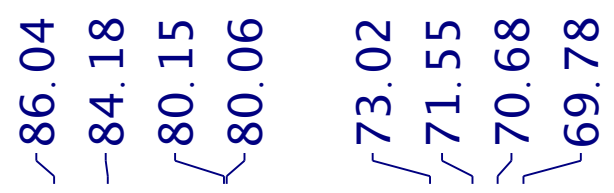

न

$m \sim$

$\sim \sim$

$\underbrace{\sim}$

เก

$\stackrel{0}{\rightarrow-1}$
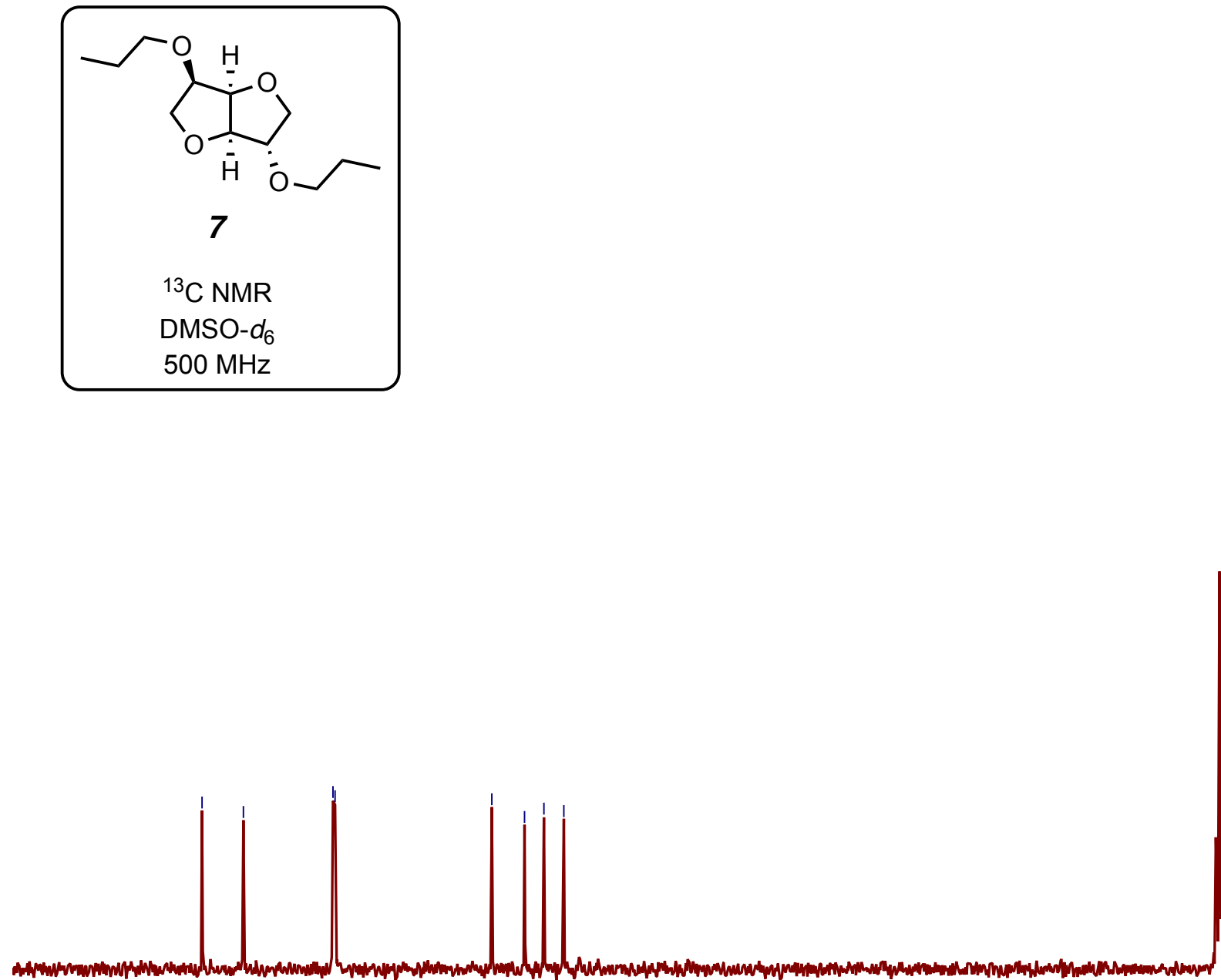

11

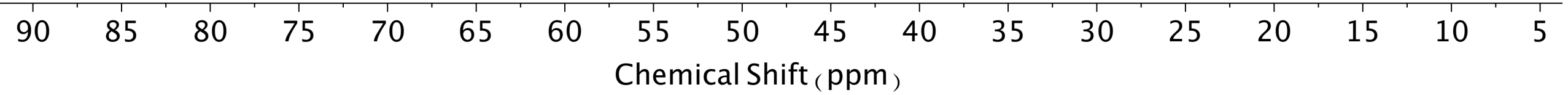




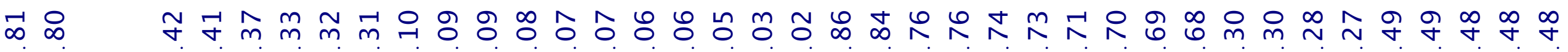

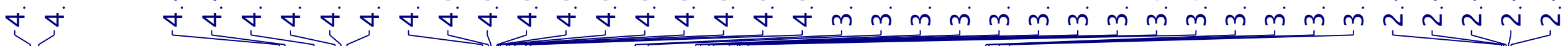
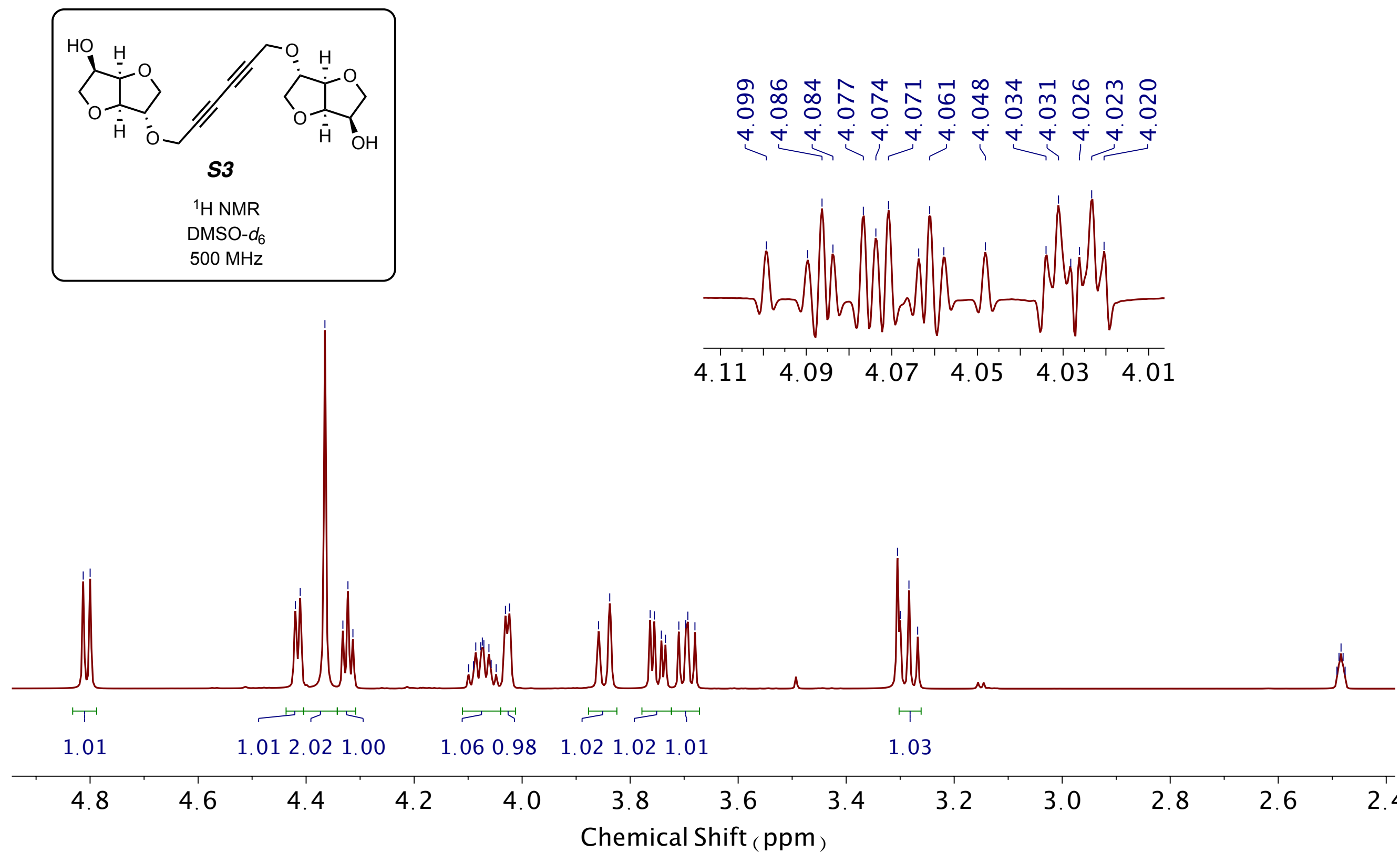


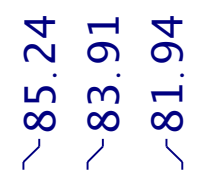

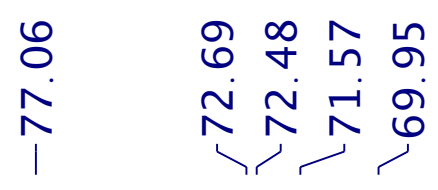
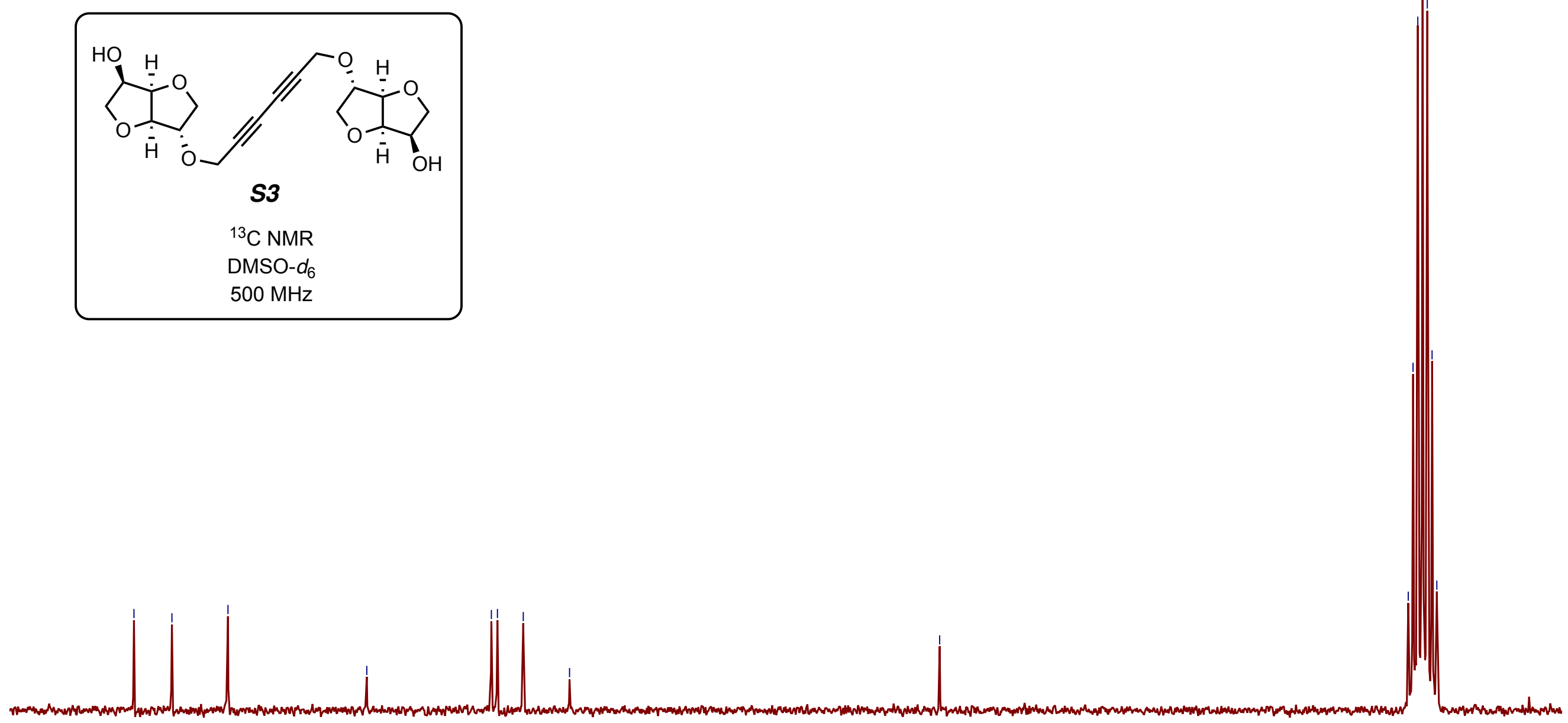
กิ ナ

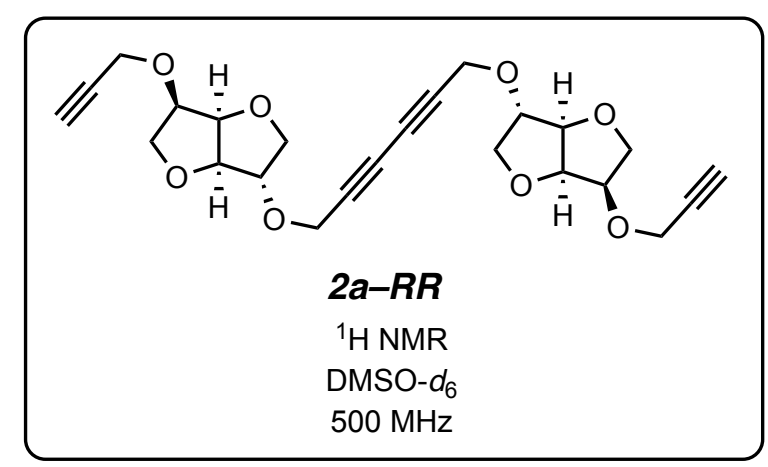

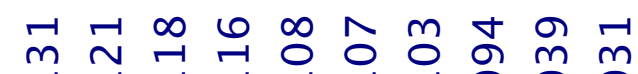

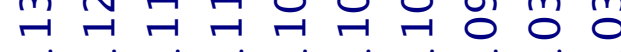

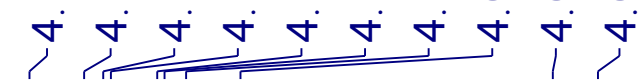
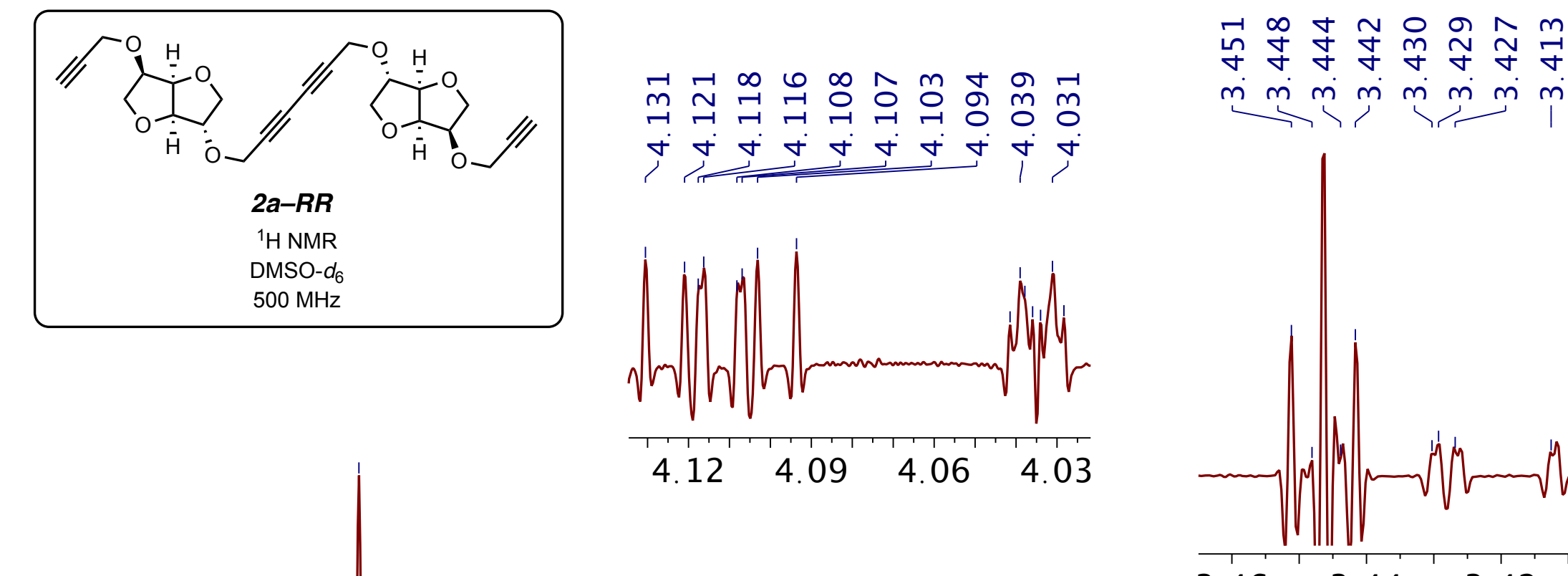

$\begin{array}{llll}4.12 & 4.09 & 4.06 & 4.03\end{array}$
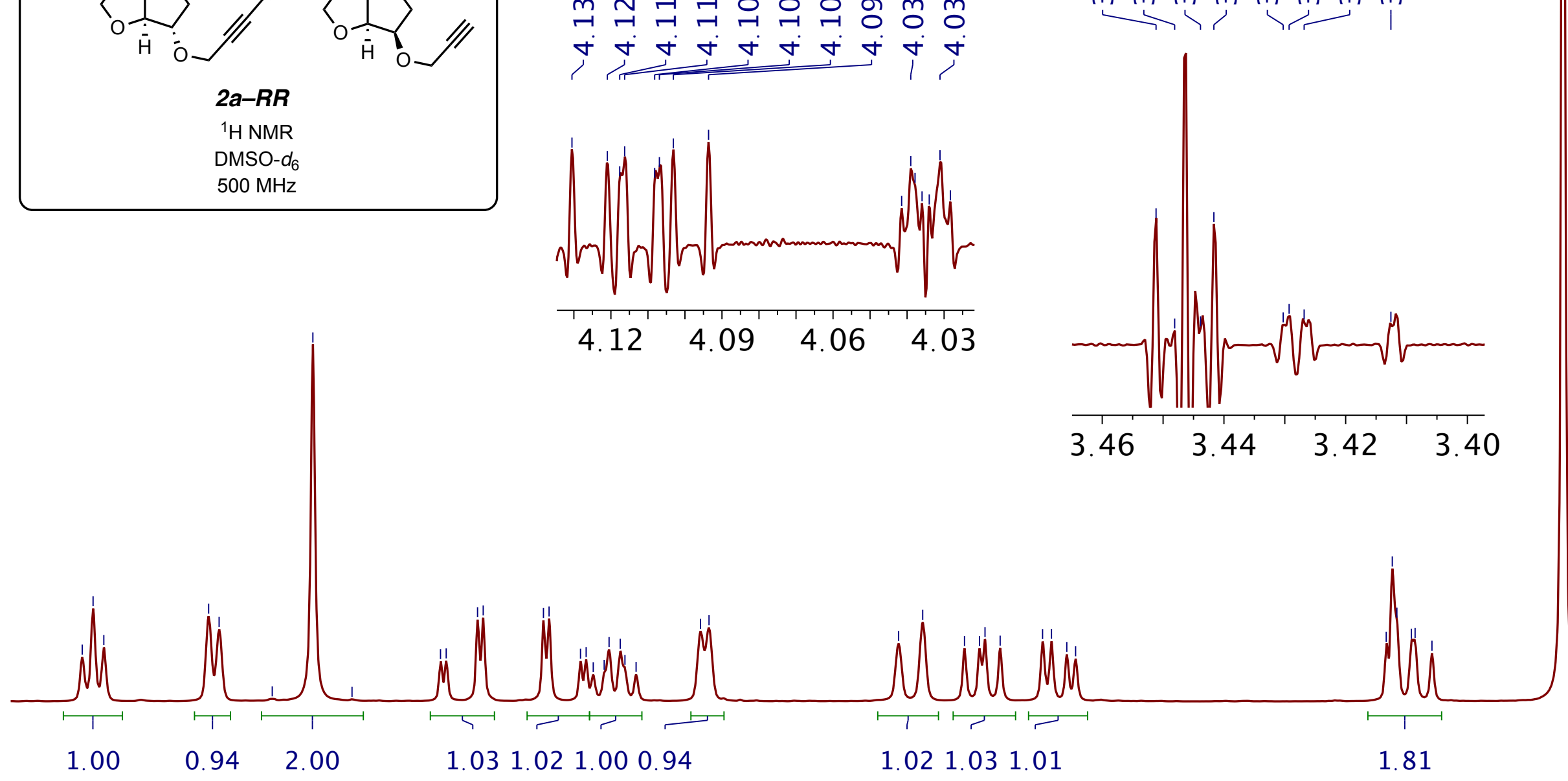

\begin{tabular}{|c|c|c|c|c|c|c|c|c|c|c|c|c|c|c|}
\hline 4.6 & 4.5 & 4.4 & 4.3 & 4.2 & 4.1 & 4.0 & 3.9 & 3.8 & 3.7 & 3.6 & 3.5 & 3.4 & 3.3 & 3.2 \\
\hline & & & & & & Che & al Shi & ppm ) & & & & & & \\
\hline
\end{tabular}



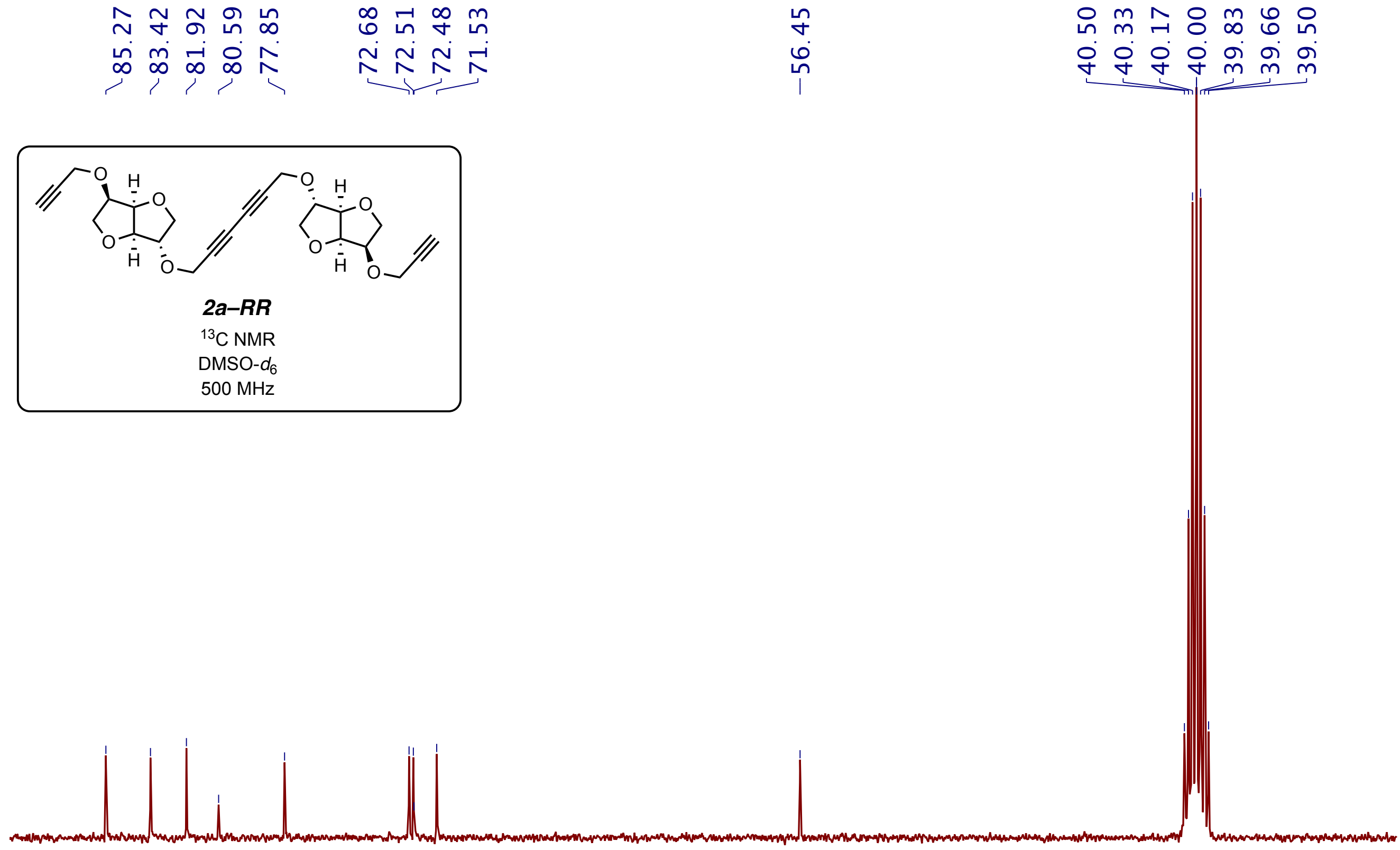


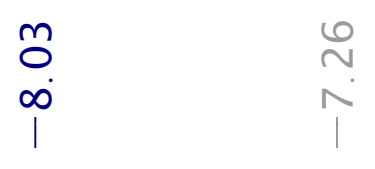

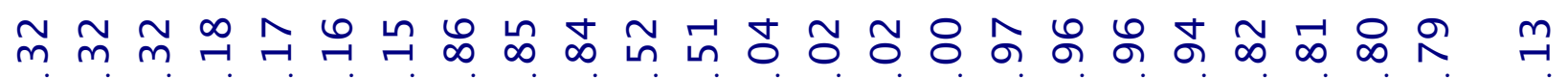

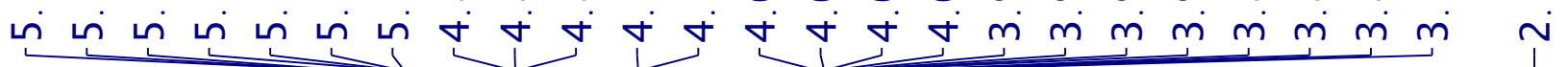
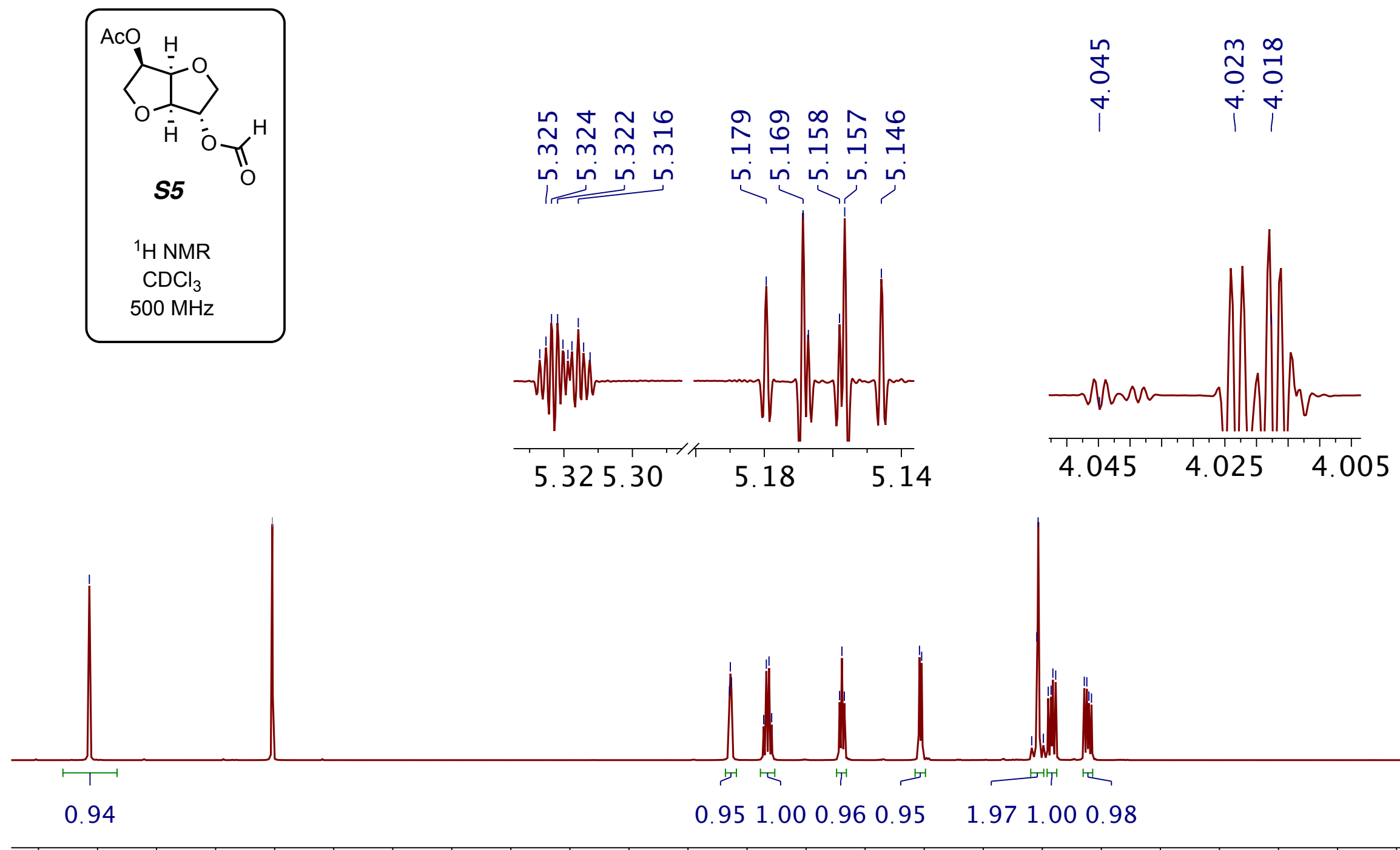

$\begin{array}{llllllll}8.0 & 7.5 & 7.0 & 6.5 & 6.0 & \begin{array}{l}5.5 \\ \text { Chemical Shift }(\mathrm{ppm})\end{array}\end{array}$



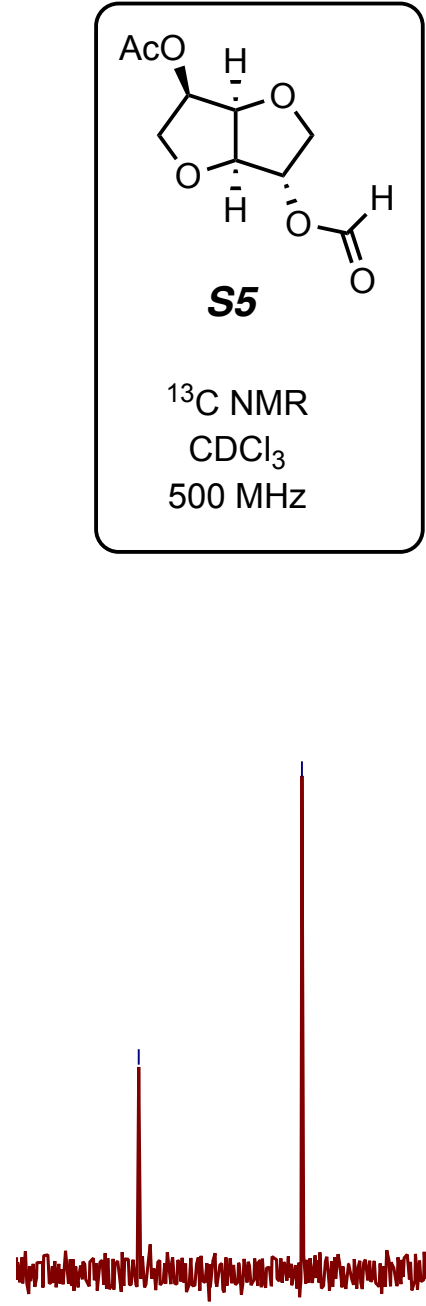

$170 \quad 160$ 150 140
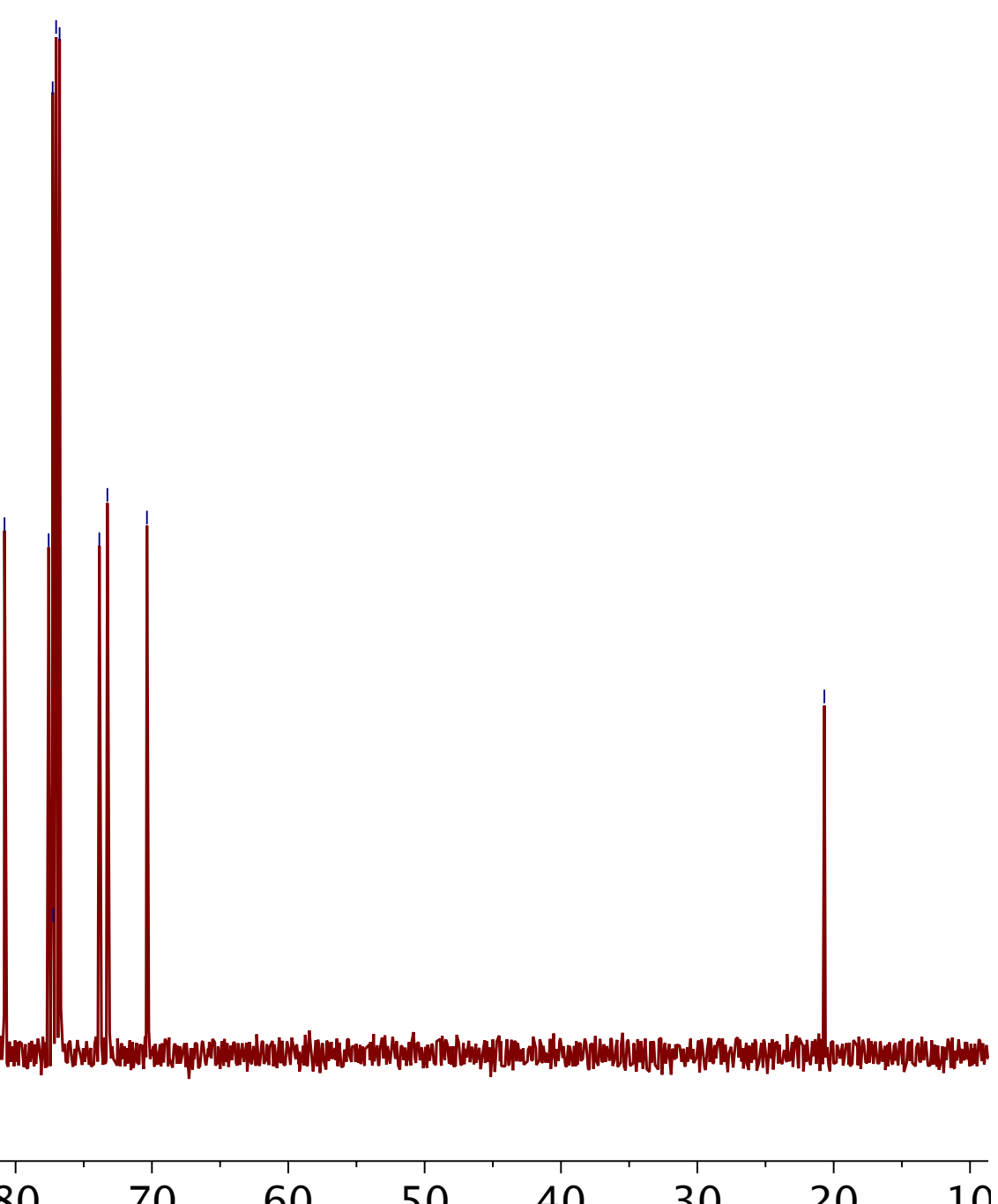


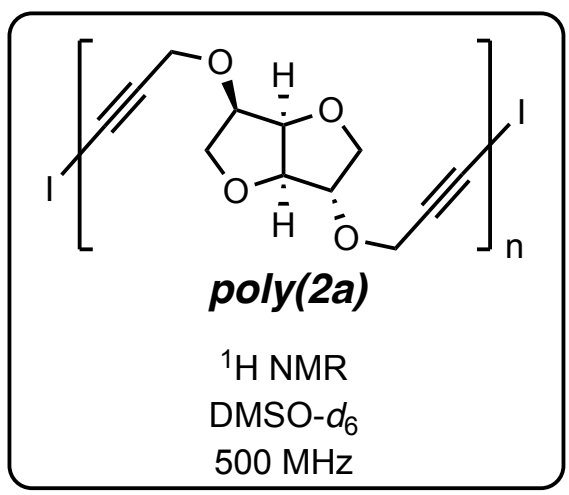

ઉં

न $\rightarrow$ -

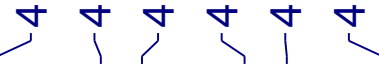

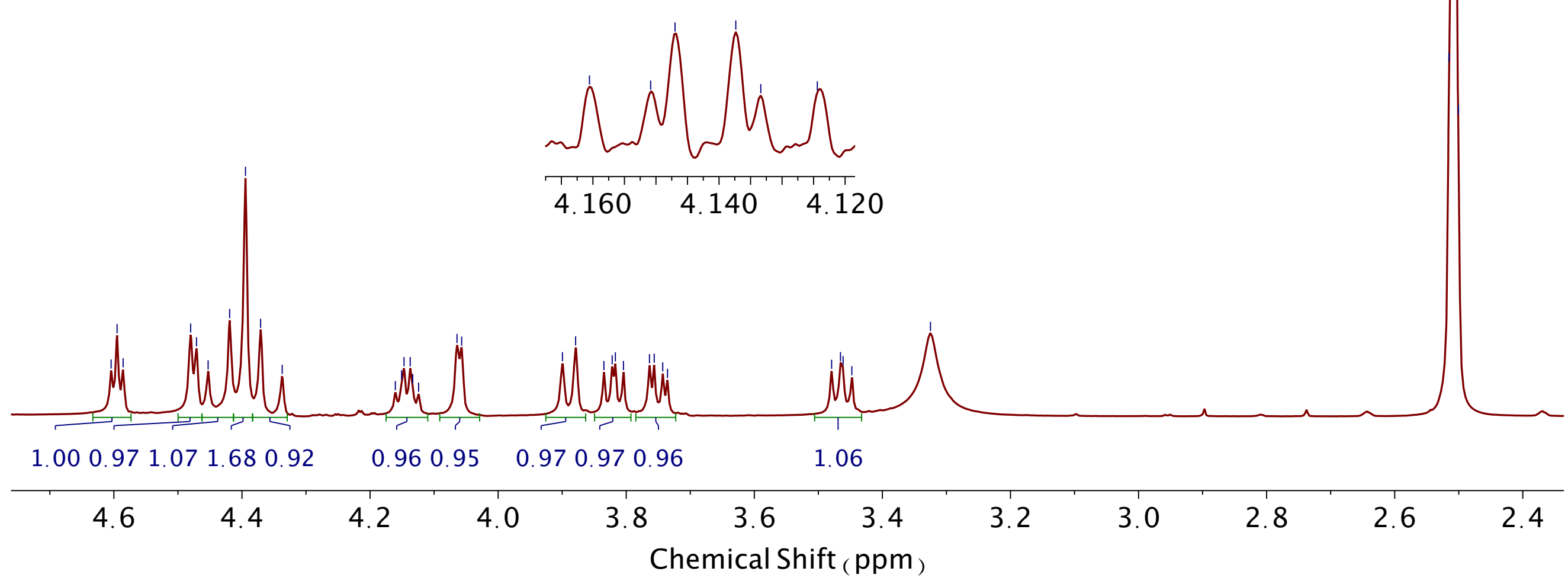




$\begin{array}{cccc}\forall & + & \stackrel{N}{\sim} \\ 0 & + & 0 \\ \infty & \infty & \infty & 0 \\ 1 & 1 & 1 & 1\end{array}$

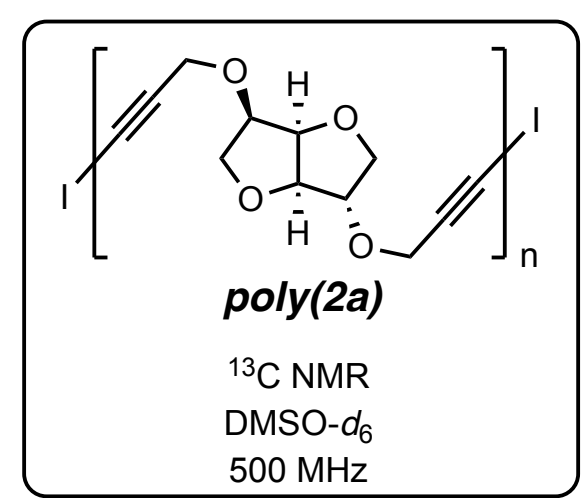

$\infty 6$

กิ
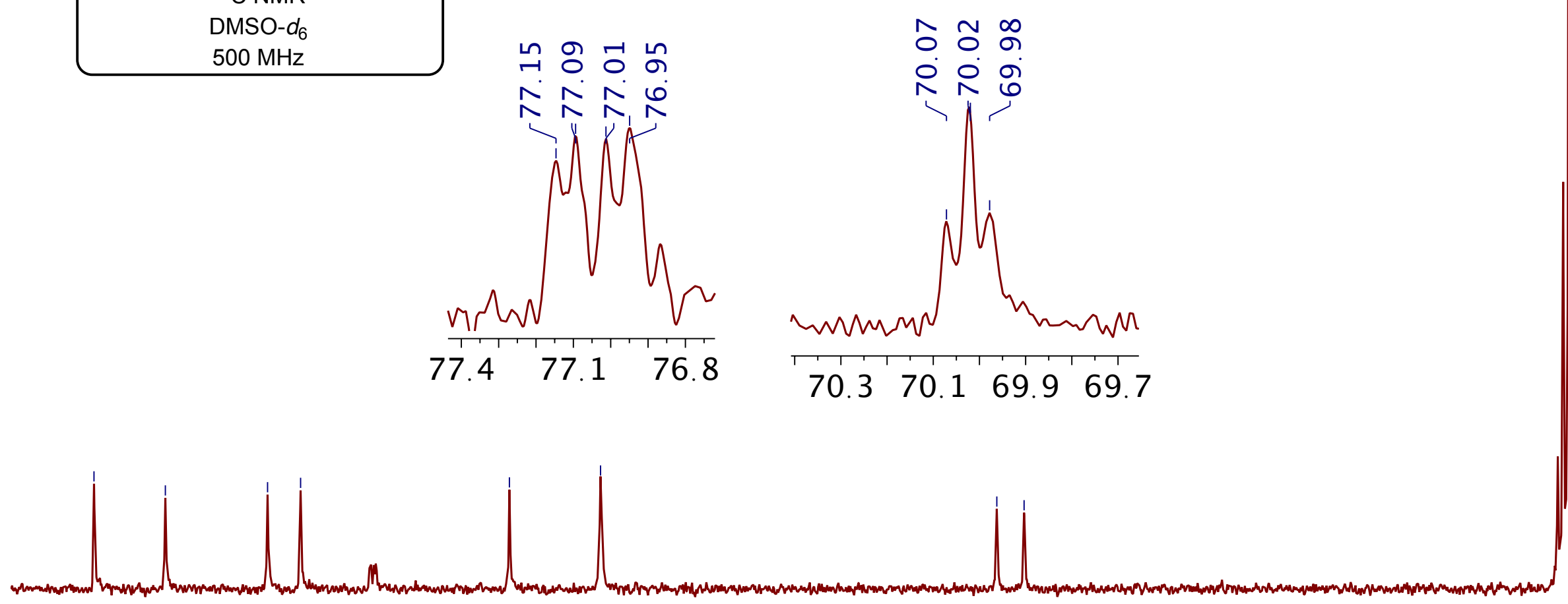

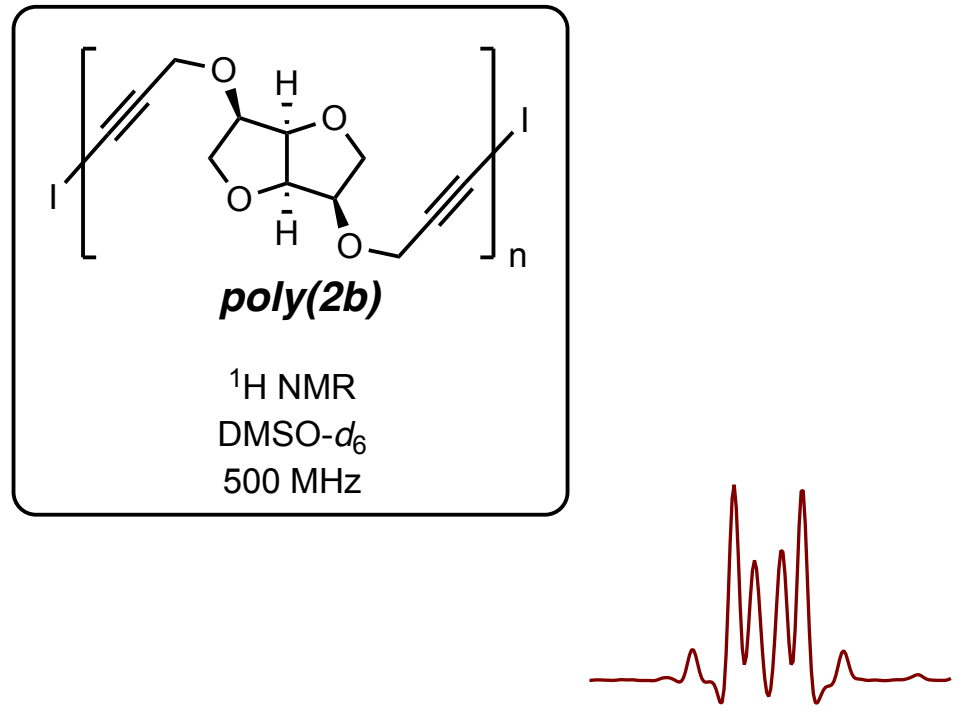

$4.520 \quad 4.495$

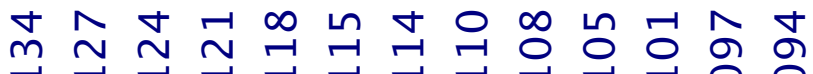

$$
\begin{aligned}
& \underbrace{\forall}
\end{aligned}
$$

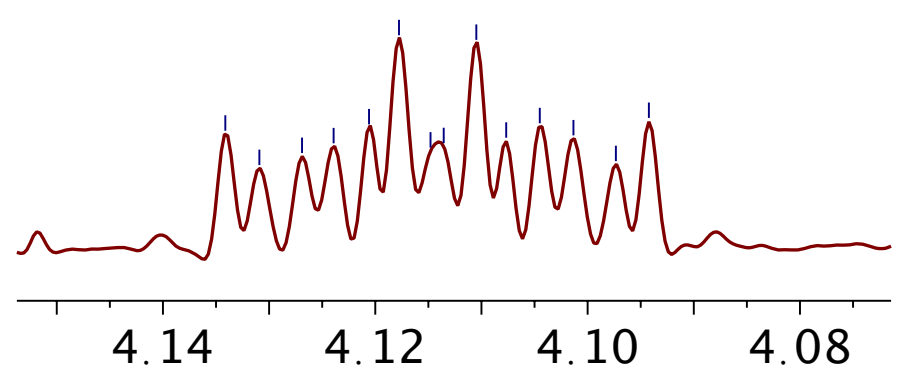

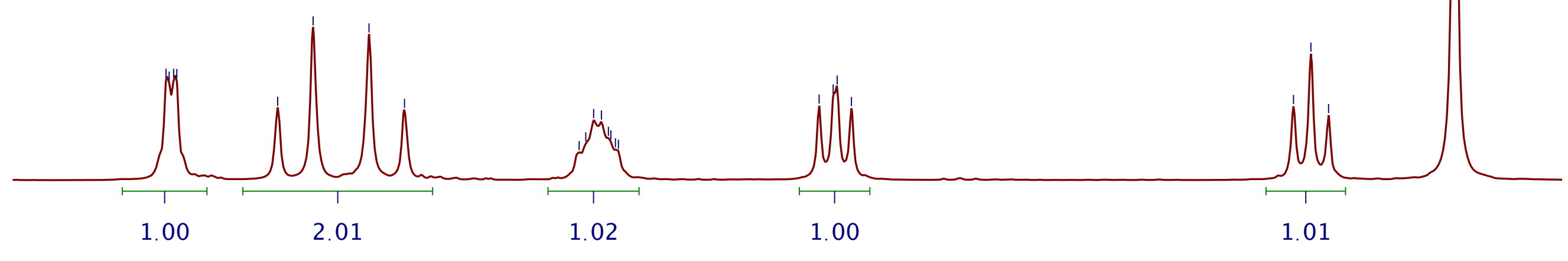

$\begin{array}{llllllllllllll}4.6 & 4.5 & 4.4 & 4.3 & 4.2 & 4.1 & \begin{array}{c}4.0 \\ \text { Chemical Shift }(\mathrm{ppm})\end{array} & \begin{array}{c}3.9 \\ 3.8\end{array} & 3.7 & 3.6 & 3.5 & 3.4 & 3.3\end{array}$




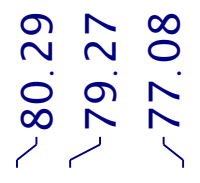

$\begin{array}{ll}0 & 0 \\ 0 & \\ 0 & 0 \\ 1 & 1\end{array}$

กิ

다

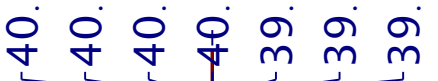
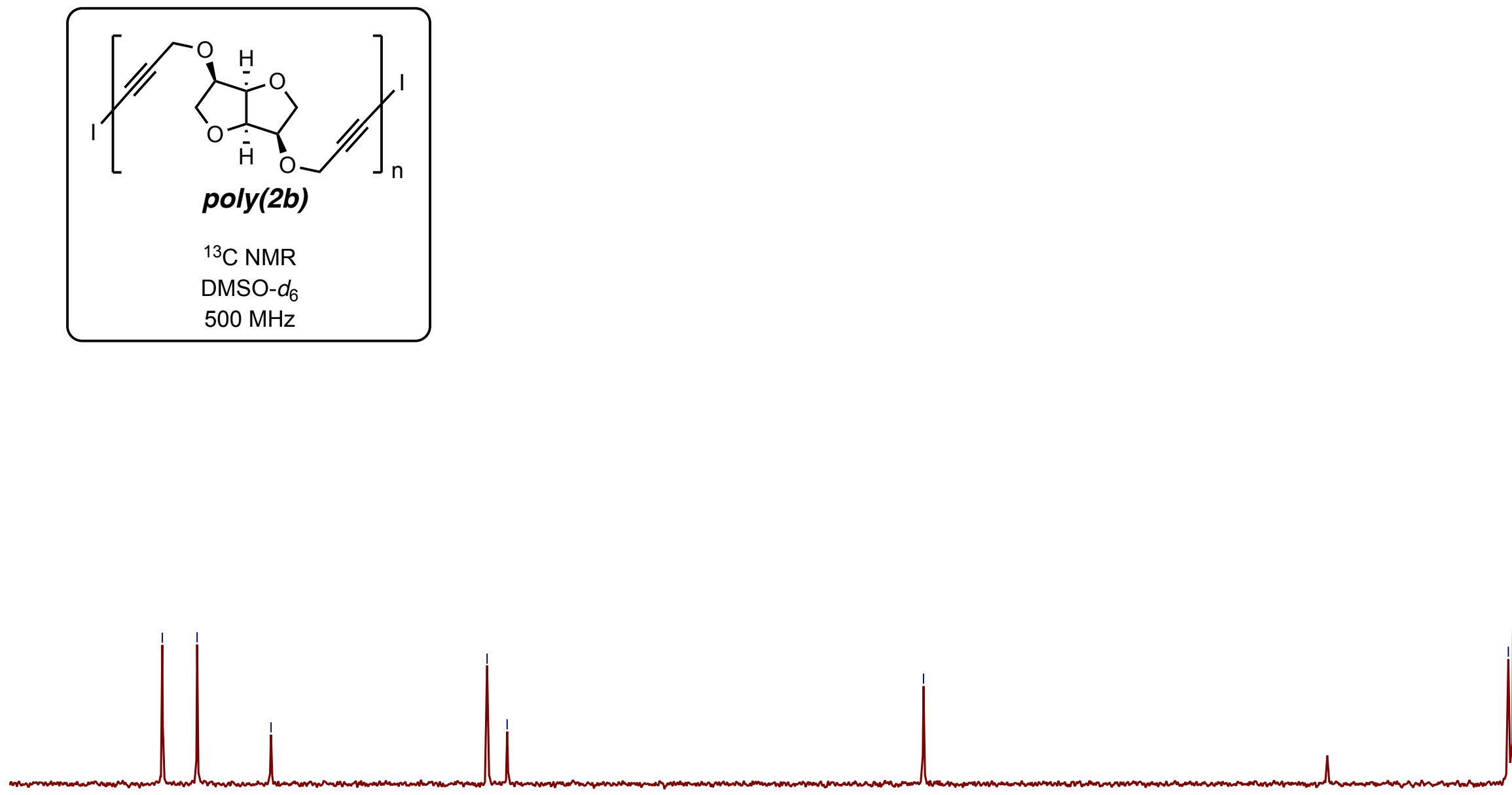

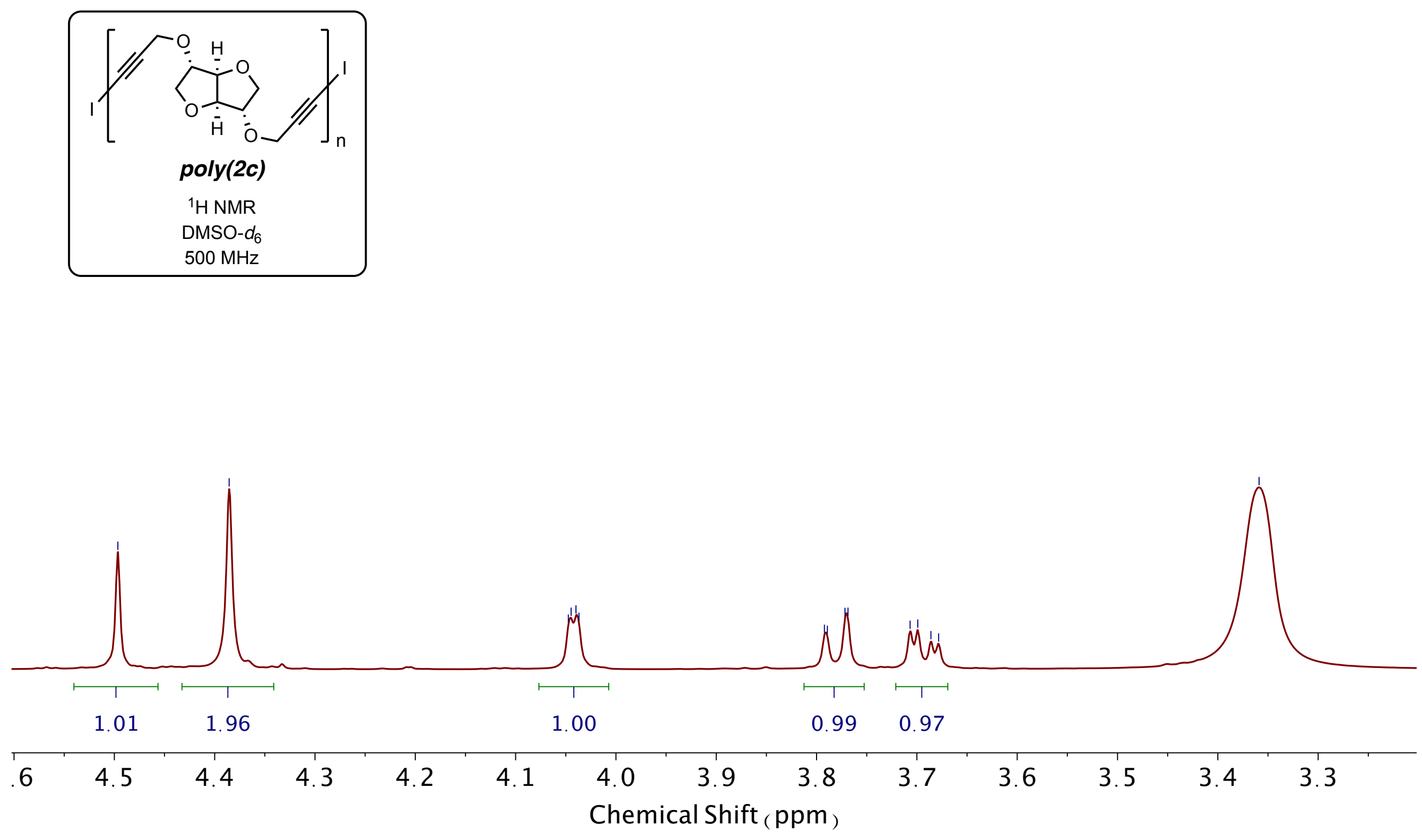


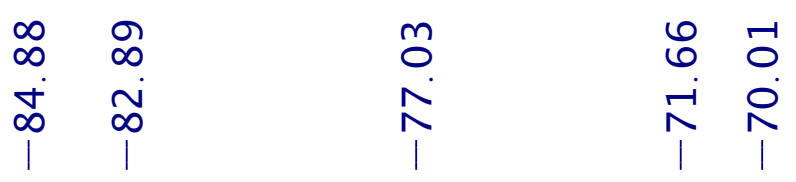

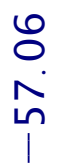

$m$
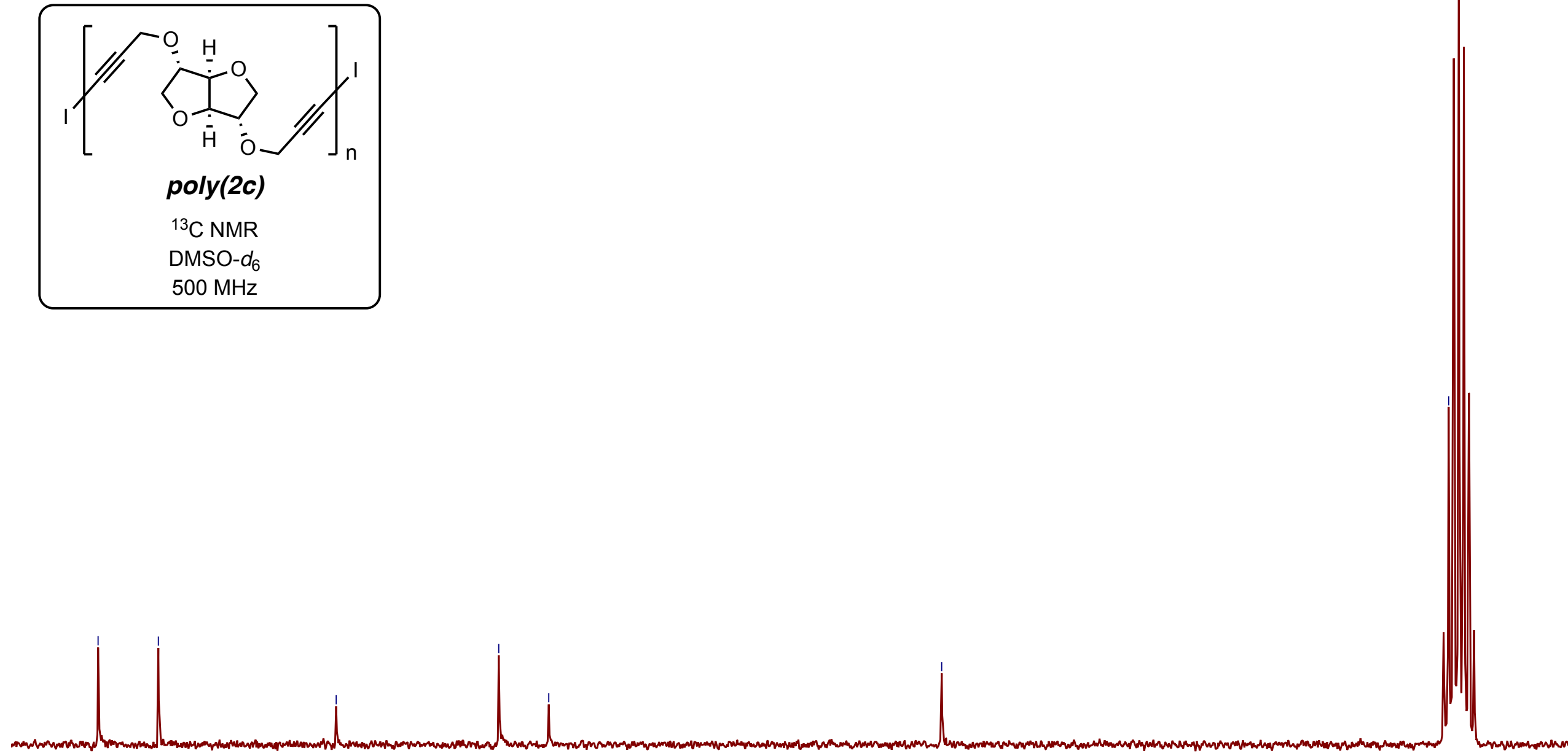

Chemical Shift (ppm) 
ค

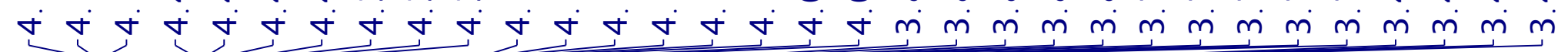

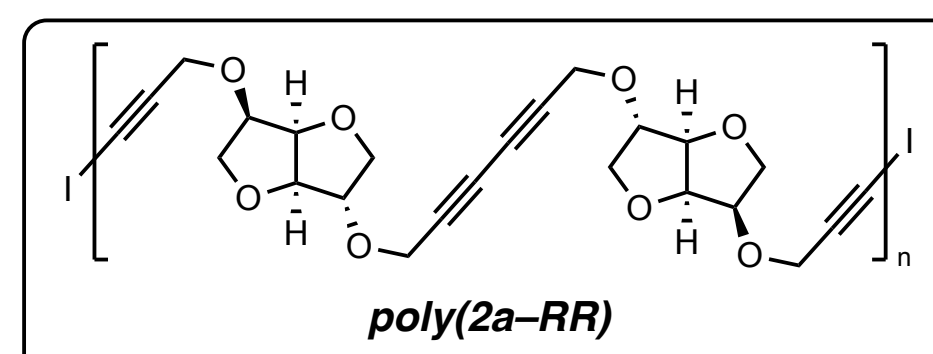

${ }^{1} \mathrm{H}$ NMR DMSO- $d_{6}$ $500 \mathrm{MHz}$

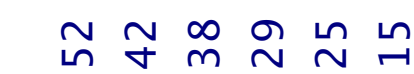

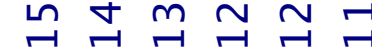

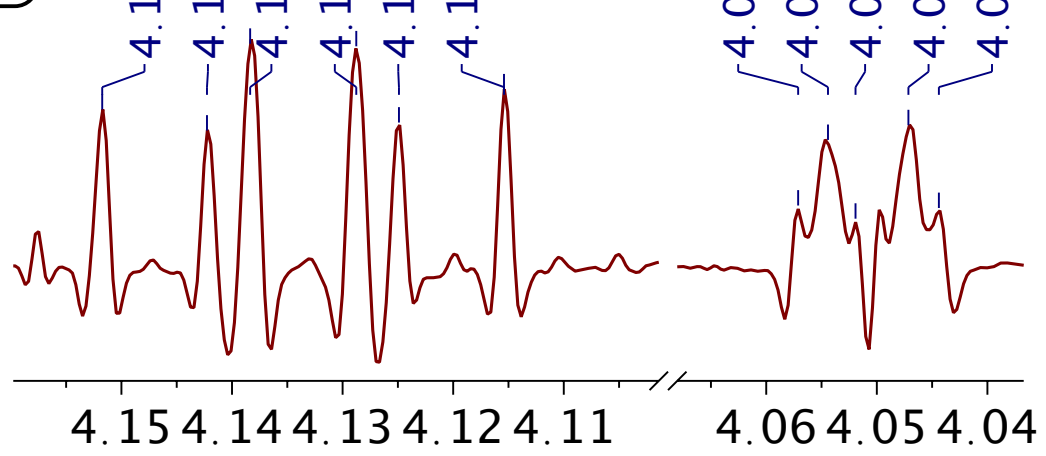

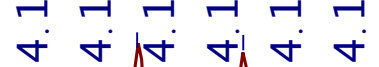
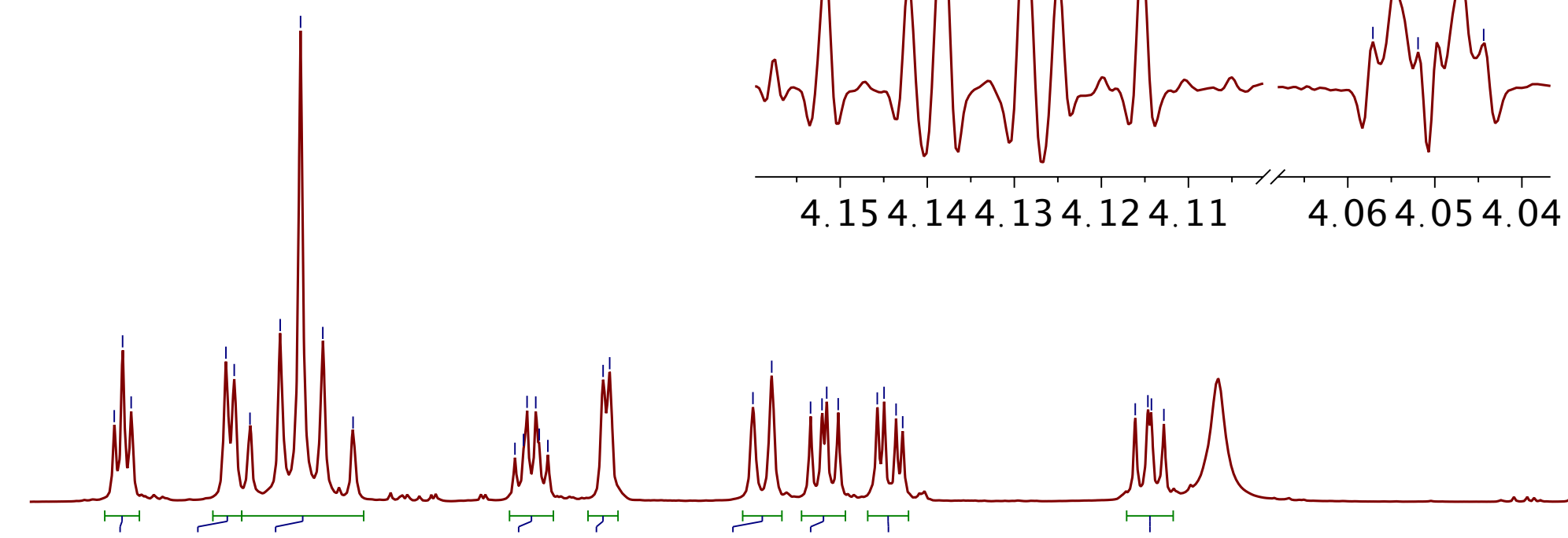

1.001 .044 .21
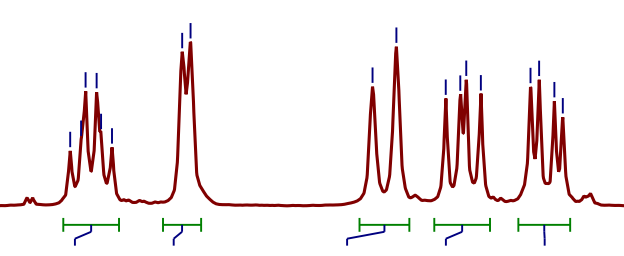

$1.031 .02 \quad 1.031 .061 .04$

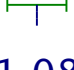

$\begin{array}{lllllllllllllllllllllllll}4.6 & 4.5 & 4.4 & 4.3 & 4.2 & 4.1 & 4.0 & 3.9 & 3.8 & 3.7 & 3.6 & 3.5 & 3.4 & 3.3 & 3.2 & 3.1 & 3.0 & 2.9 & 2.8 & 2.7 & 2.6 & 2.5\end{array}$ Chemical Shift (ppm) 


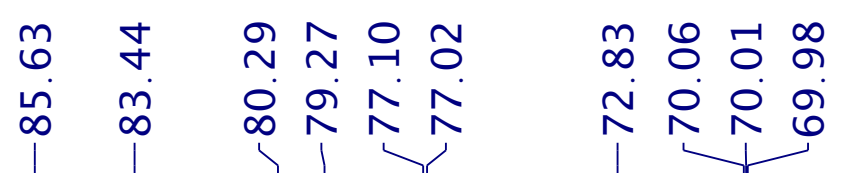

-

in

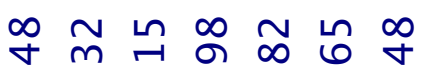

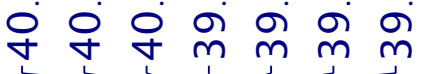
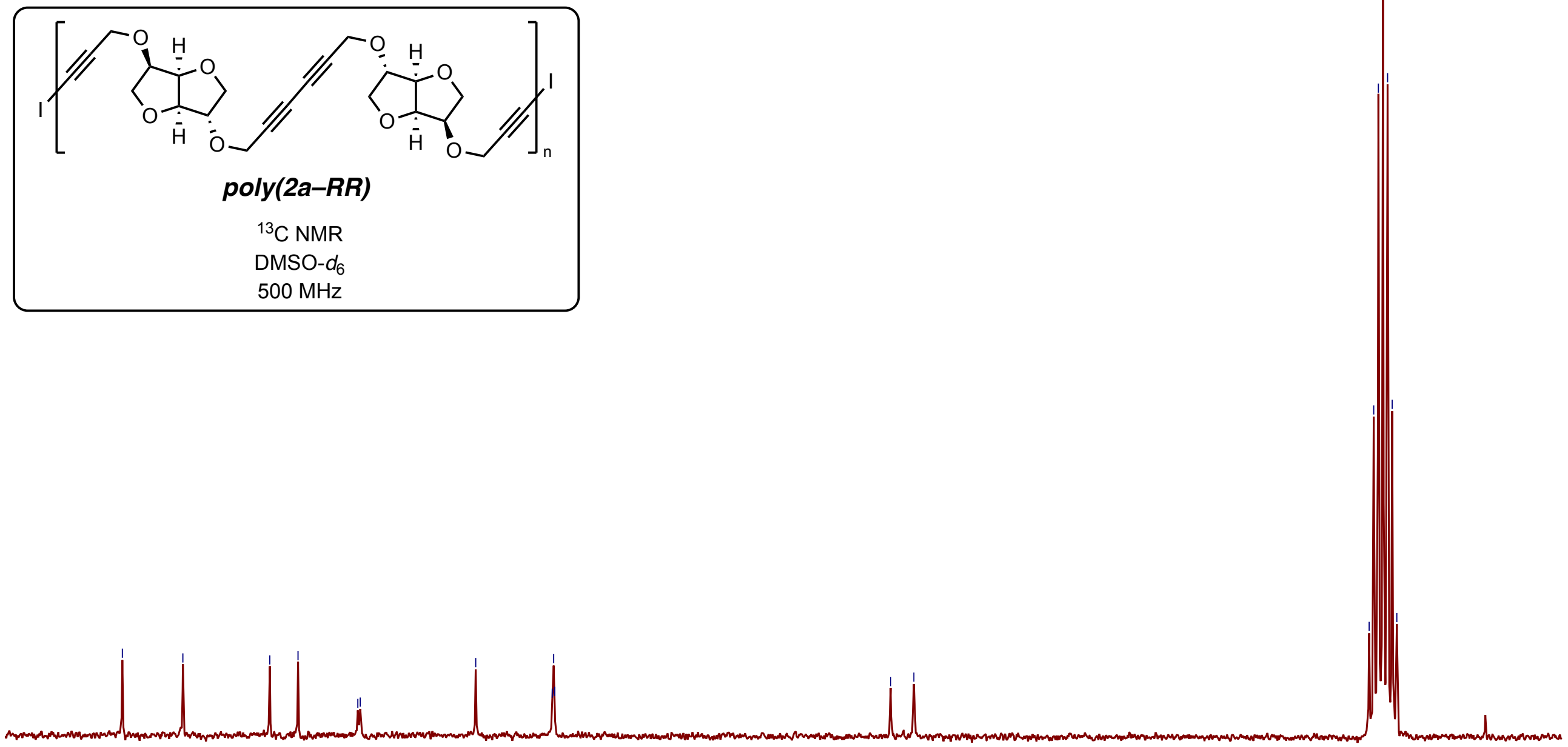

$\begin{array}{llllllllllllllllllllllllllll}88 & 86 & 84 & 82 & 80 & 78 & 76 & 74 & 72 & 70 & 68 & 66 & 64 & 62 & 60 & 58 & 56 & 54 & 52 & 50 & 48 & 46 & 44 & 42 & 40 & 38 & 36 & 34\end{array}$ Chemical Shift ( $\mathrm{ppm}$ ) 


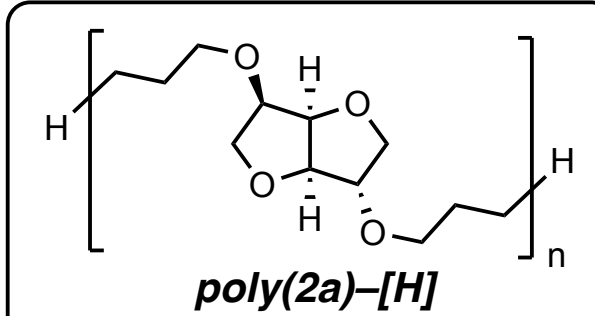

${ }^{1} \mathrm{H}$ NMR DMSO- $d_{6}$ $500 \mathrm{MHz}$
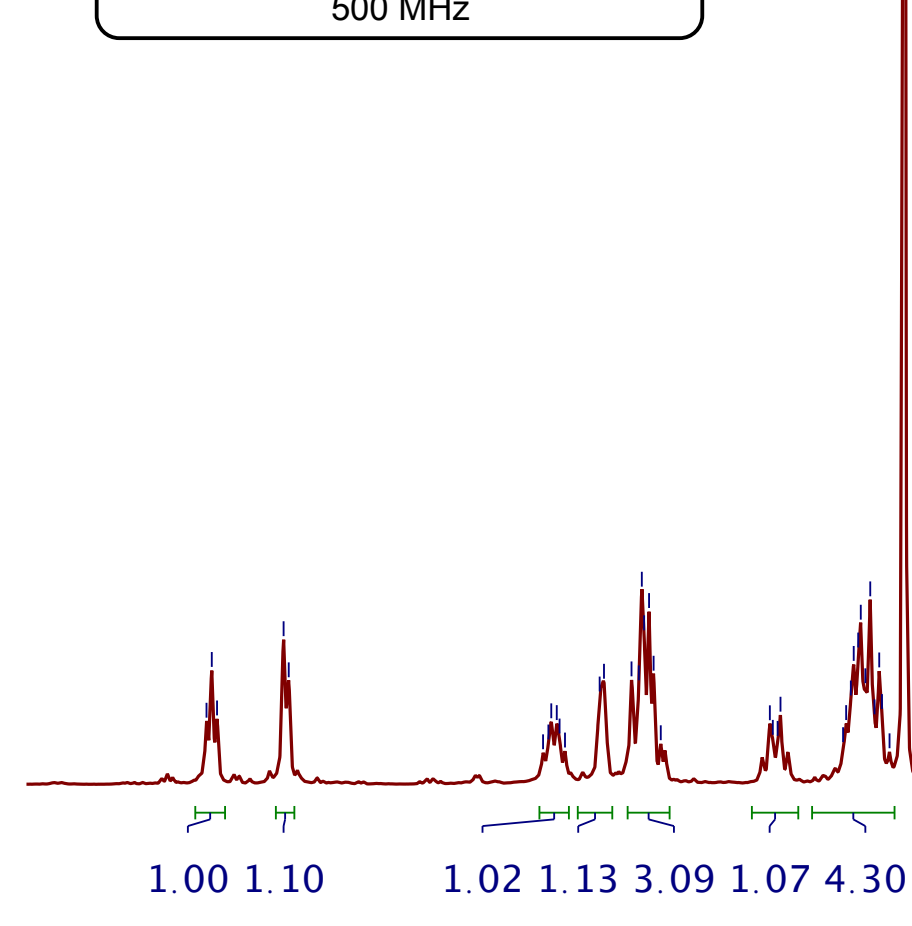

4.5

3.5
3.0

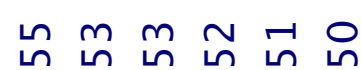
$m m m \dot{m} m$ $m m m m^{m} m$

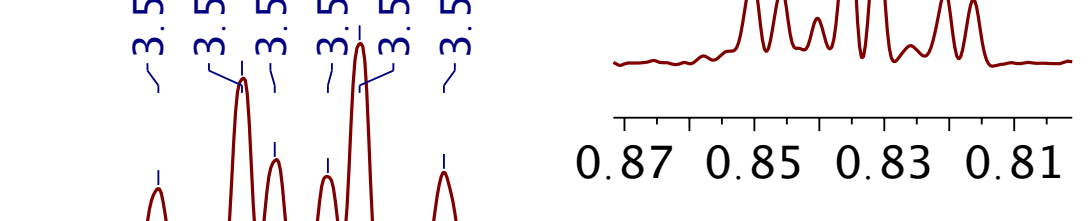

가

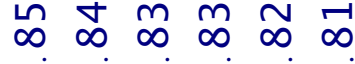

○ 00 i 000

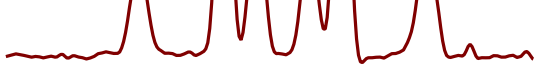

$\begin{array}{llll}3.55 & 3.53 & 3.51 & 3.49\end{array}$

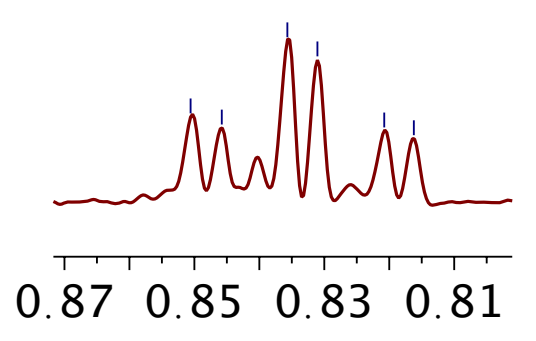

Chemical Shift (ppm) 


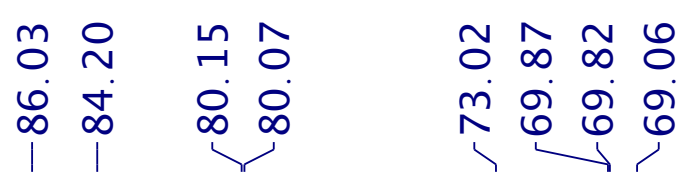

\begin{tabular}{|c|c|c|}
\hline$\stackrel{\leftrightarrow}{\forall} m \stackrel{v}{m}$ & $\underset{\infty}{\infty}$ & ڤ \\
\hline $\begin{array}{lll}0 & + & 0 \\
\end{array}$ & m & $\stackrel{\sim}{\sim}$ \\
\hline
\end{tabular}

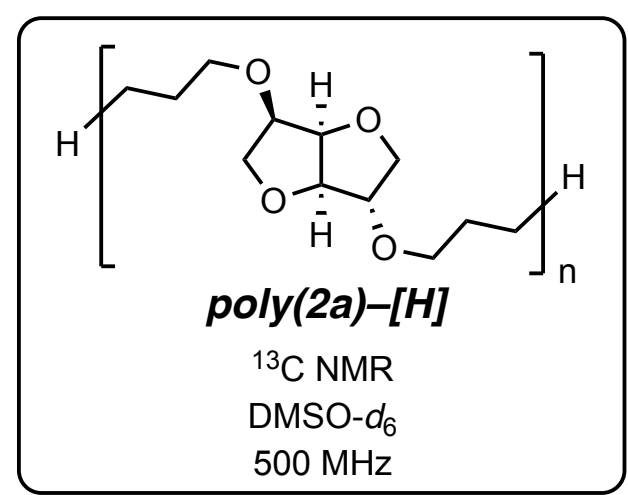

-

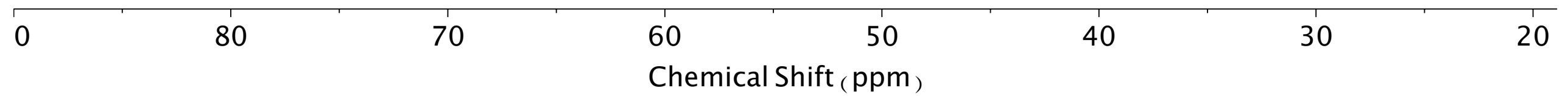



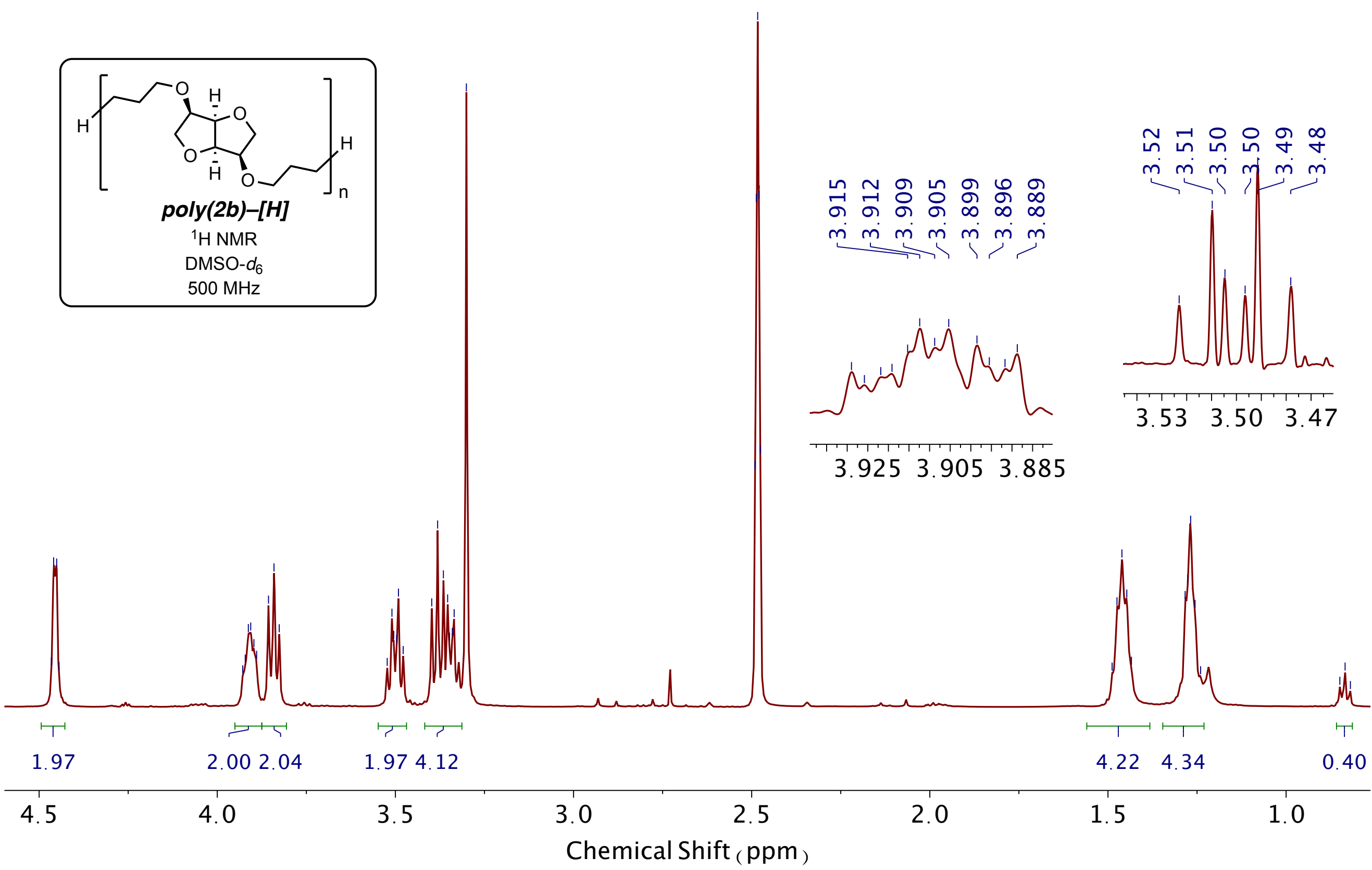


$\begin{array}{lll}\infty & \circ \\ 0 & \infty & 0 \\ \infty & 0 & 0 \\ 1 & 1 & 1\end{array}$
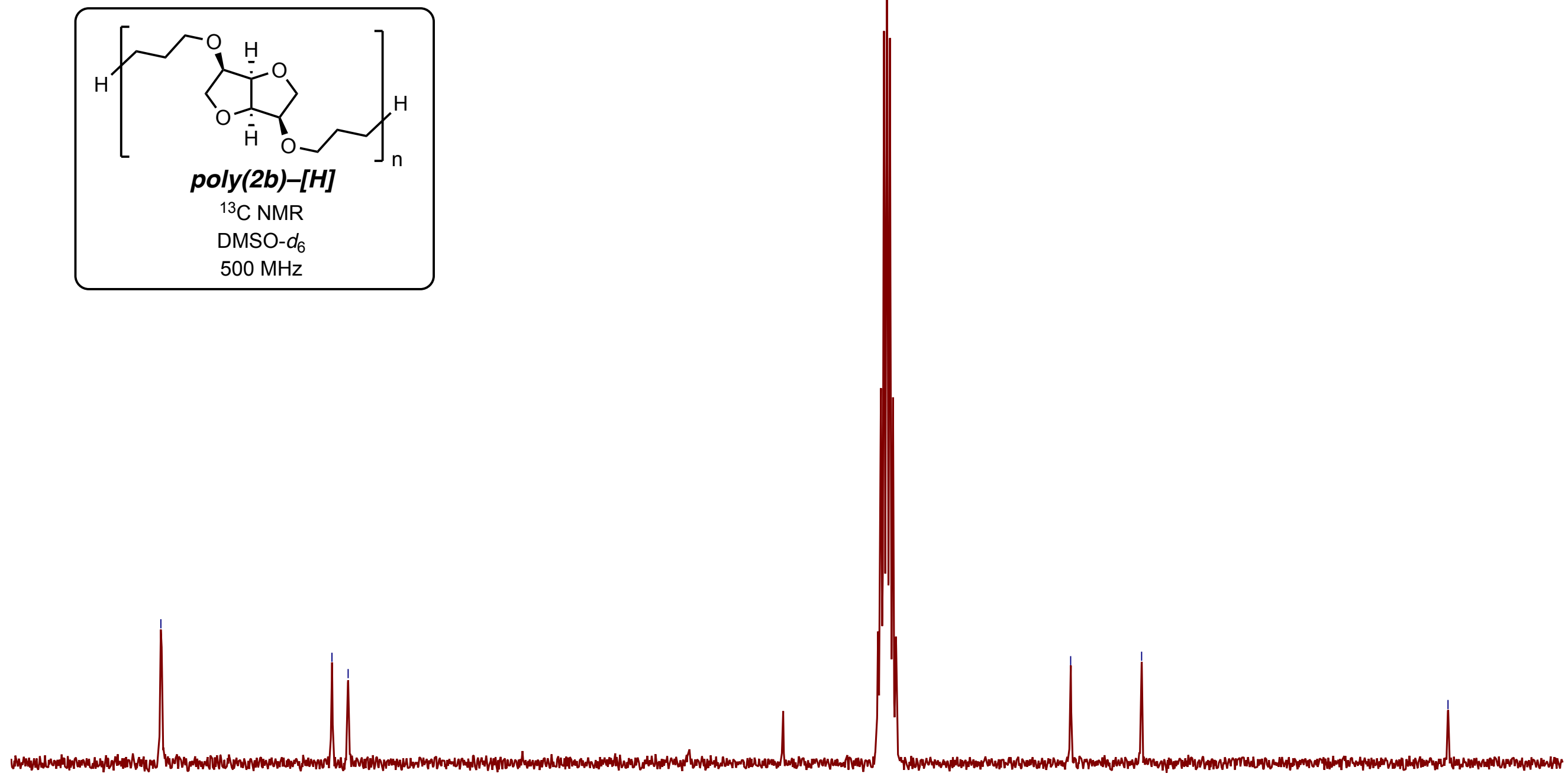


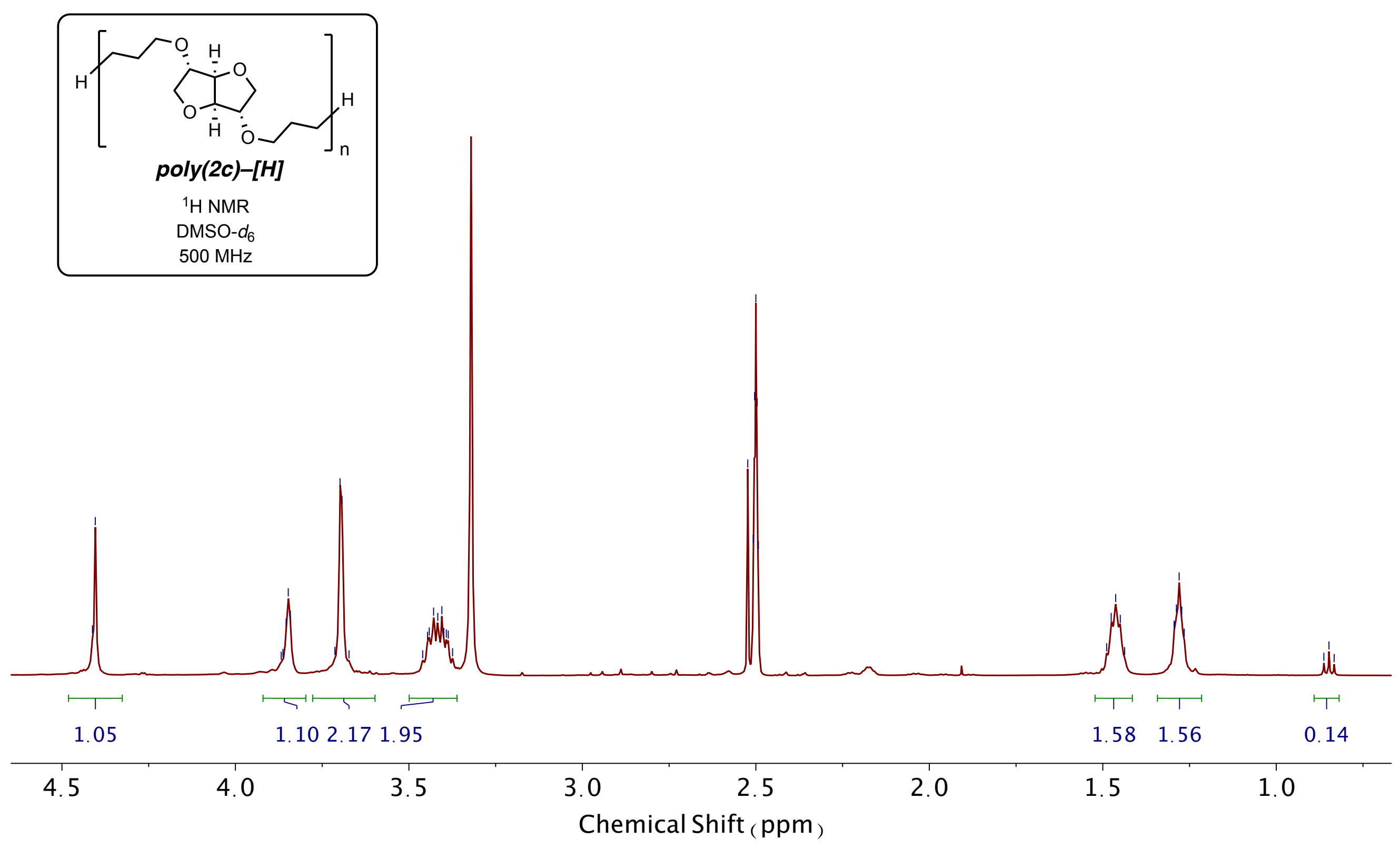




\begin{tabular}{|c|c|}
\hline$\vec{m} \stackrel{+}{\dot{y}}$ & $\underset{\infty}{+}$ \\
\hline$\stackrel{\infty}{\infty} \dot{m}^{\infty}$ & ז \\
\hline
\end{tabular}
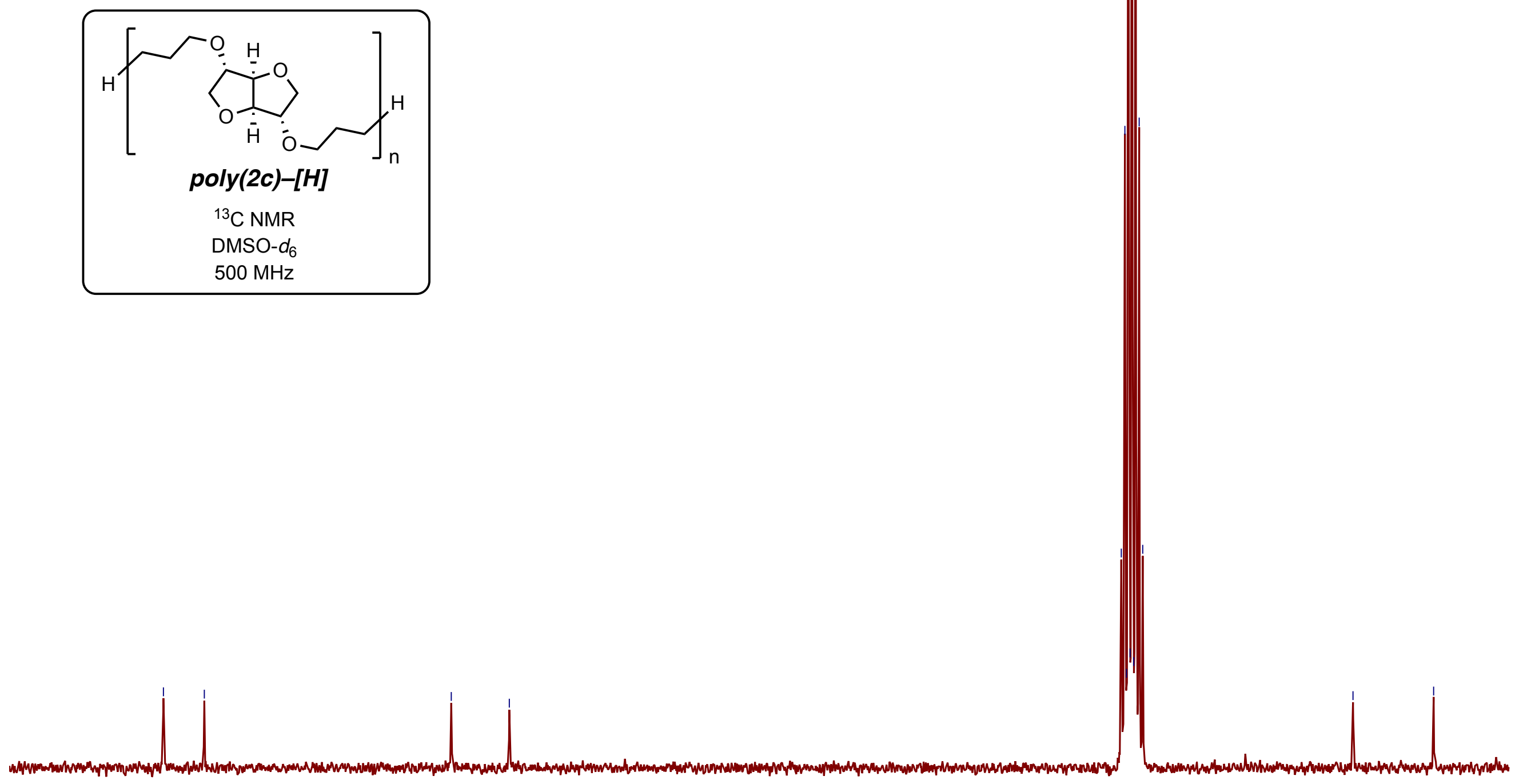